\title{
Enantioselective Syntheses of (Z)-6'-Boryl-anti-1,2-oxaborinan-3-enes via a Dienylboronate Protoboration and Asymmetric Allylation Reaction Sequence
}

Jichao Chen and Ming Chen*

Department of Chemistry and Biochemistry

Auburn University

E-mail: mzc0102@auburn.edu

Supporting Information: Experimental Procedures, Tabulated Spectroscopic Data, ${ }^{1} \mathrm{H}$ and

${ }^{13} \mathrm{C}$ Spectra of New Compounds 
General Experimental Details. All reaction solvents were purified before use. Dichloromethane, 1,2-dichloroethane and toluene were purified by passing through a solvent column composed of activated A-1 alumina. Unless indicated otherwise, all reactions were conducted under an atmosphere of argon using flame-dried or oven-dried $\left(120{ }^{\circ} \mathrm{C}\right)$ glassware. The term "concentrated under reduced pressure" refers to the removal of solvents and other volatile materials using a rotary evaporator with the water bath temperature below $30{ }^{\circ} \mathrm{C}$, followed by the removal of residual solvents at high vacuum $(<0.2$ mbar $)$.

Proton nuclear magnetic resonance ( ${ }^{1} \mathrm{H}$ NMR) spectra were acquired on commercial instruments (400 and $600 \mathrm{MHz}$ ) at Auburn University NMR facility. Carbon-13 nuclear magnetic resonance $\left({ }^{13} \mathrm{C}\right.$ NMR) spectra were acquired at 101 and $151 \mathrm{MHz}$. The proton signal for the residual non-deuterated solvent $\left(\delta 7.26\right.$ for $\left.\mathrm{CHCl}_{3}\right)$ was used as an internal reference for ${ }^{1} \mathrm{H}$ NMR spectra. For ${ }^{13} \mathrm{C}$ NMR spectra, chemical shifts are reported relative to the $\delta 77.36$ resonance of $\mathrm{CHCl}_{3}$. Coupling constants are reported in $\mathrm{Hz}$. High-resolution mass spectra were recorded on a commercial high-resolution mass spectrometer (mass analyzer type: QTOF) via the Micro Mass/Analytical Facility operated by the College of Chemistry and Biochemistry, Auburn University.

Analytical thin layer chromatography (TLC) was performed on Kieselgel 60 F254 glass plates precoated with a $0.25 \mathrm{~mm}$ thickness of silica gel. The TLC plates were visualized with UV light and/or by staining with Hanessian solution (ceric sulfate and ammonium molybdate in aqueous sulfuric acid) or $\mathrm{KMnO}_{4}$. Column chromatography was generally performed using Kieselgel 60 (230-400 mesh) silica gel, typically using a 50-100:1 weight ratio of silica gel to crude product. 


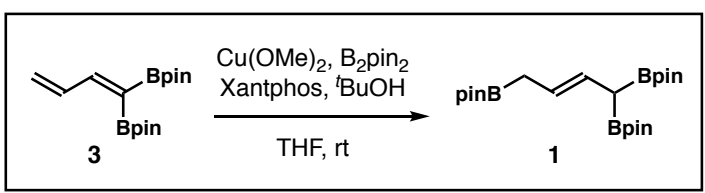

(E)-2,2' ,2' -(But-2-ene-1,1,4-triyl)tris(4,4,5,5-tetramethyl-1,3,2-dioxaborolane) (1): In an Ar-filled glove box, $\mathrm{Cu}(\mathrm{OMe})_{2}(1 \mathrm{mg}, 0.01 \mathrm{mmol}, 10 \mathrm{~mol} \%)$, Xantphos (6 mg, 0.01 mmol, $10 \mathrm{~mol} \%)$, anhydrous THF $(0.5 \mathrm{~mL})$ and a Teflon-coated magnetic stirring bar were sequentially added into a reaction vial. The mixture was stirred at ambient temperature for $15 \mathrm{~min}$. $\mathrm{B}_{2} \mathrm{Pin}_{2}(31 \mathrm{mg}, 0.12 \mathrm{mmol}, 1.2$ equiv) was added and the resulting mixture was stirred for another $5 \mathrm{~min}$. Then 1,1-bisboryl-1,3-butadiene $\mathbf{3}^{1}$ (31 $\mathrm{mg}, 0.1 \mathrm{mmol}, 1.0$ equiv) and ${ }^{t} \mathrm{BuOH}(8 \mathrm{mg}, 0.1 \mathrm{mmol}, 1.0$ equiv) were sequentially added to the mixture. After stirring for $10 \mathrm{~min}$, hexane $(2 \mathrm{~mL})$ was added to the reaction and the resulting mixture was filtered through a pad of Celite. The solvent was removed under reduced pressure. Purification of the crude product by column chromatography (hexane : ethyl acetate $=7.5: 1$ ) gave product 1 as white solid in $74 \%$ yield $(32 \mathrm{mg}$ ). When the reaction was run in a 1 mmol scale, 1 was isolated in $71 \%$ yield $(310 \mathrm{mg}) .{ }^{1} \mathrm{H}$ NMR (400 MHz, $\left.\mathrm{CDCl}_{3}\right) \delta 5.51(\mathrm{dd}, J=15.2,9.1 \mathrm{~Hz}, 1 \mathrm{H}), 5.33(\mathrm{dt}, J=15.0,7.3 \mathrm{~Hz}$, $1 \mathrm{H}), 1.76(\mathrm{~d}, J=8.9 \mathrm{~Hz}, 1 \mathrm{H}), 1.63(\mathrm{~d}, J=7.2 \mathrm{~Hz}, 2 \mathrm{H}), 1.21(\mathrm{~s}, 36 \mathrm{H}) .{ }^{13} \mathrm{C}$ NMR $(151$ $\left.\mathrm{MHz} \mathrm{CDCl}_{3}\right) \delta 126.2,124.2,83.4,83.3,25.1,25.0,24.9,16.7$. HRMS $\left(\mathrm{ESI}^{+}\right): \mathrm{m} / \mathrm{z}$ for $\mathrm{C}_{22} \mathrm{H}_{41} \mathrm{~B}_{3} \mathrm{O}_{6} \mathrm{Na}[\mathrm{M}+\mathrm{Na}]^{+}$calcd 457.3080, found 457.3081.

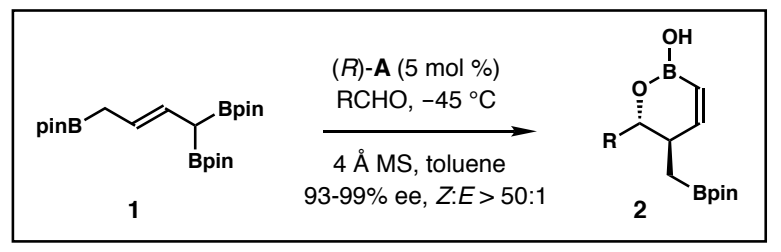

General procedure for the syntheses of $(Z)-6$ '-Boryl-anti-1,2-oxaborinan-3-enes 2:

To a reaction vial containing a stirring bar and freshly activated $4 \AA$ MS (50 mg) was added chiral phosphoric acid $(R)$-A (4 mg, $0.005 \mathrm{mmol}, 5 \mathrm{~mol} \%)$. Toluene $(0.3 \mathrm{~mL})$ was added to the vial followed by addition of freshly distilled aldehyde $(0.12 \mathrm{mmol}, 1.2$ equiv, if it is a liquid). The mixture was placed in a $-45^{\circ} \mathrm{C}$ cold bath and stirred for $5 \mathrm{~min}$. Then a solution of allylboronate 1 (44 mg, $0.1 \mathrm{mmol}, 1.0$ equiv) in toluene ( $0.2 \mathrm{~mL}$ ) was added slowly to the reaction mixture via a microliter syringe. The mixture was kept stirring at $45{ }^{\circ} \mathrm{C}$ for $48 \mathrm{~h}$. After complete consumption of the aldehyde, the reaction mixture was filtered through a pad of silica gel. The filtrate was concentrated under reduced pressure. Purification of the crude product was performed by column chromatography (hexane and 
ethyl acetate) to give product $\mathbf{2}$. The enantiopurity of $\mathbf{2}$ was determined by HPLC analysis of the diphenylation product derived from Suzuki coupling with $\mathrm{PhI}$.

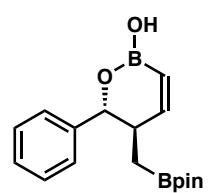

(5R,6R)-6-Phenyl-5-((4,4,5,5-tetramethyl-1,3,2-dioxaborolan-2-yl)meth yl)-5,6-dihydro-2H-1,2-oxaborinin-2-ol (2a) Prepared according to the general procedure. The crude mixture was purified by flash column chromatography (hexane : ethyl acetate $=5: 1$ ) to give the title compound as colorless oil in 95\% yield (30 mg). Enantiomeric excess was determined by HPLC analysis of the corresponding diphenylation product to be $98 \%$ ee $\left(254 \mathrm{~nm}, 25^{\circ} \mathrm{C}\right) ; \mathrm{t}_{1}=$ $6.64 \mathrm{~min}, \mathrm{t}_{2}=8.05 \mathrm{~min}\left[(\right.$ Chiralpak IE) hexane $/ \mathrm{i}-\mathrm{PrOH}, 95: 5,1.0 \mathrm{~mL} / \mathrm{min}] ;[\alpha]_{\mathrm{D}}{ }^{20}=$ $+15.2^{\circ}\left(\mathrm{c} 0.25, \mathrm{CHCl}_{3}\right) ;{ }^{1} \mathrm{H} \mathrm{NMR}\left(400 \mathrm{MHz}, \mathrm{CDCl}_{3}\right) \delta 7.29-7.36(\mathrm{~m}, 5 \mathrm{H}), 6.82(\mathrm{~d}, J=$ $12.1 \mathrm{~Hz}, 1 \mathrm{H}), 5.74$ (d, $J=12.3 \mathrm{~Hz}, 1 \mathrm{H}), 4.77$ (d, $J=9.5 \mathrm{~Hz}, 1 \mathrm{H}), 4.13$ (s, 1H), 2.73 $2.79(\mathrm{~m}, 1 \mathrm{H}), 1.21(\mathrm{~s}, 12 \mathrm{H}), 0.82(\mathrm{dd}, J=15.9,5.1 \mathrm{~Hz}, 1 \mathrm{H}), 0.73(\mathrm{dd}, J=15.9,9.7 \mathrm{~Hz}$, $1 \mathrm{H}) .{ }^{13} \mathrm{C}$ NMR $\left(151 \mathrm{MHz}, \mathrm{CDCl}_{3}\right) \delta 157.3,142.2,128.6,128.2,127.6,83.7,82.8,41.1$, 25.3, 25.1, 14.4. HRMS (ESI $\left.{ }^{+}\right): m / z$ for $\mathrm{C}_{17} \mathrm{H}_{24} \mathrm{~B}_{2} \mathrm{O}_{4} \mathrm{Na}[\mathrm{M}+\mathrm{Na}]^{+}$calcd 337.1758, found 337.1769. A 1 mmol-scale reaction was also conducted and $2 \mathbf{a}$ was isolated in $86 \%$ yield (270 mg).

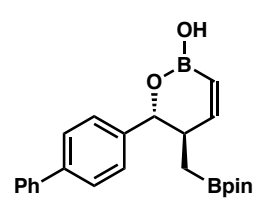

$(5 R, 6 R)-6-([1,1 '-B i p h e n y l]-4-y l)-5-((4,4,5,5-t e t r a m e t h y l-1,3,2-d i o x a b o$ rolan-2-yl)methyl)-5,6-dihydro-2H-1,2-oxaborinin-2-ol (2b) Prepared according to the general procedure. The crude mixture was purified by flash column chromatography (hexane : ethyl acetate $=5: 1$ ) to give the title compound as a white solid in $82 \%$ yield $(32 \mathrm{mg})$. Enantiomeric excess was determined by HPLC analysis of the corresponding diphenylation product to be $99 \%$ ee $\left(254 \mathrm{~nm}, 25^{\circ} \mathrm{C}\right) ; \mathrm{t}_{1}=13.8 \mathrm{~min}, \mathrm{t}_{2}=16.8 \mathrm{~min}$ [(Chiralpak IA) hexane/i-PrOH, 95:5, 1.0 $\mathrm{mL} / \mathrm{min}] ;[\alpha]_{\mathrm{D}}{ }^{20}=+32.4^{\circ}\left(\mathrm{c} 0.50, \mathrm{CHCl}_{3}\right) ;{ }^{1} \mathrm{H} \mathrm{NMR}\left(400 \mathrm{MHz}, \mathrm{CDCl}_{3}\right) \delta 7.57-7.59(\mathrm{~m}$, $4 \mathrm{H}), 7.42-7.45(\mathrm{~m}, 4 \mathrm{H}), 7.32-7.36(\mathrm{~m}, 1 \mathrm{H}), 6.85(\mathrm{~d}, J=12.0 \mathrm{~Hz}, 1 \mathrm{H}), 5.77(\mathrm{~d}, J=12.1$ $\mathrm{Hz}, 1 \mathrm{H}), 4.82(\mathrm{~d}, J=9.5 \mathrm{~Hz}, 1 \mathrm{H}), 4.11(\mathrm{~s}, 1 \mathrm{H}), 2.78-2.84(\mathrm{~m}, 1 \mathrm{H}), 1.21(\mathrm{~s}, 12 \mathrm{H}), 0.88$ $(\mathrm{dd}, J=15.7,5.1 \mathrm{~Hz}, 1 \mathrm{H}), 0.78(\mathrm{dd}, J=16.0,9.6 \mathrm{~Hz}, 1 \mathrm{H}) .{ }^{13} \mathrm{C} \mathrm{NMR}\left(101 \mathrm{MHz}, \mathrm{CDCl}_{3}\right)$ $\delta 157.3,141.24,141.18,141.08,129.1,128.0,127.6,127.4$ (two overlapping C signals), 83.7, 82.6, 41.0, 25.3, 25.1. HRMS $\left(\mathrm{ESI}^{+}\right): \mathrm{m} / z$ for $\mathrm{C}_{23} \mathrm{H}_{28} \mathrm{~B}_{2} \mathrm{O}_{4} \mathrm{Na}[\mathrm{M}+\mathrm{Na}]^{+}$calcd 413.2071, found 413.2065.

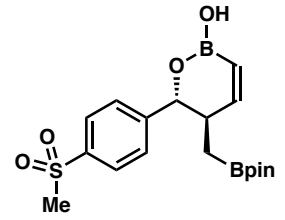

(5R,6R)-6-(4-(Methylsulfonyl)phenyl)-5-((4,4,5,5-tetramethyl-1,3,2dioxaborolan-2-yl)methyl)-5,6-dihydro-2H-1,2-oxaborinin-2-ol (2c)

Prepared according to the general procedure. The crude mixture was 
purified by flash column chromatography (hexane : ethyl acetate $=1: 1$ ) to give the title compound as a white solid in $84 \%$ yield $(33 \mathrm{mg}$ ). Enantiomeric excess was determined by HPLC analysis of the corresponding diphenylation product to be $99 \%$ ee $(254 \mathrm{~nm}$, $\left.25^{\circ} \mathrm{C}\right) ; \mathrm{t}_{1}=16.0 \mathrm{~min}, \mathrm{t}_{2}=17.0 \mathrm{~min}$ [(Chiralpak IA) hexane $/ \mathrm{i}-\mathrm{PrOH}, 90: 10,1.0 \mathrm{~mL} / \mathrm{min}$; $[\alpha]_{\mathrm{D}}^{20}=+22.4^{\circ}\left(\mathrm{c} 0.25, \mathrm{CHCl}_{3}\right) ;{ }^{1} \mathrm{H} \mathrm{NMR}\left(400 \mathrm{MHz}, \mathrm{CDCl}_{3}\right) \delta 7.93(\mathrm{~d}, J=8.3 \mathrm{~Hz}, 2 \mathrm{H})$, $7.58(\mathrm{~d}, J=8.2 \mathrm{~Hz}, 2 \mathrm{H}), 6.80(\mathrm{~d}, J=12.6 \mathrm{~Hz}, 1 \mathrm{H}), 5.76(\mathrm{~d}, J=12.2 \mathrm{~Hz}, 1 \mathrm{H}), 4.90(\mathrm{~d}, J=$ $9.1 \mathrm{~Hz}, 1 \mathrm{H}), 4.18(\mathrm{~s}, 1 \mathrm{H}), 3.05(\mathrm{~s}, 3 \mathrm{H}), 2.69-2.75(\mathrm{~m}, 1 \mathrm{H}), 1.22(\mathrm{~s}, 12 \mathrm{H}), 0.73-0.85(\mathrm{~m}$, $2 \mathrm{H}) .{ }^{13} \mathrm{C}$ NMR $\left(151 \mathrm{MHz}, \mathrm{CDCl}_{3}\right) \delta 156.7,148.6,140.1,128.5,127.7,83.9,81.7,44.9$, 41.2, 25.3, 25.1, 14.5. HRMS $\left(\mathrm{ESI}^{+}\right): m / z$ for $\mathrm{C}_{18} \mathrm{H}_{26} \mathrm{~B}_{2} \mathrm{O}_{6} \mathrm{SNa}[\mathrm{M}+\mathrm{Na}]^{+}$calcd 415.1534, found 415.1518 .

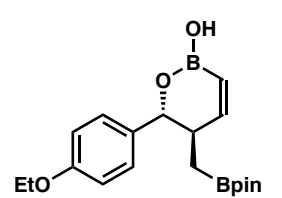

(5R,6R)-6-(4-Ethoxyphenyl)-5-((4,4,5,5-tetramethyl-1,3,2-dioxaborol an-2-yl)methyl)-5,6-dihydro-2H-1,2-oxaborinin-2-ol (2d) Prepared according to the general procedure. The crude mixture was purified by column chromatography (hexane : ethyl acetate $=5: 1$ ) to give the title compound as colorless oil in 92\% yield (33 mg). Enantiomeric excess was determined by HPLC analysis of the corresponding diphenylation product to be $99 \%$ ee $\left(254 \mathrm{~nm}, 25^{\circ} \mathrm{C}\right)$; $\mathrm{t}_{1}=12.8 \mathrm{~min}, \mathrm{t}_{2}=14.7 \mathrm{~min}[($ Chiralpak IA $)$ hexane $/ \mathrm{i}-\mathrm{PrOH}, 95: 5,1.0 \mathrm{~mL} / \mathrm{min}] ;[\alpha]_{\mathrm{D}}{ }^{20}=$ $+60.0^{\circ}\left(\mathrm{c} 0.35, \mathrm{CHCl}_{3}\right) ;{ }^{1} \mathrm{H} \mathrm{NMR}\left(400 \mathrm{MHz}, \mathrm{CDCl}_{3}\right) \delta 7.27(\mathrm{~d}, J=8.0 \mathrm{~Hz}, 2 \mathrm{H}), 6.87(\mathrm{~d}, J$ $=8.5 \mathrm{~Hz}, 2 \mathrm{H}), 6.82(\mathrm{~d}, J=12.4 \mathrm{~Hz}, 1 \mathrm{H}), 5.74(\mathrm{~d}, J=12.4 \mathrm{~Hz}, 1 \mathrm{H}), 4.69(\mathrm{~d}, J=9.8 \mathrm{~Hz}$, $1 \mathrm{H}), 4.04(\mathrm{~s}, 1 \mathrm{H}), 4.03(\mathrm{q}, J=6.9 \mathrm{~Hz}, 2 \mathrm{H}), 2.70-2.76(\mathrm{~m}, 1 \mathrm{H}), 1.41(\mathrm{t}, J=7.0 \mathrm{~Hz}, 3 \mathrm{H})$, $1.21(\mathrm{~s}, 12 \mathrm{H}), 0.79(\mathrm{dd}, J=15.8,5.0 \mathrm{~Hz}, 1 \mathrm{H}), 0.69(\mathrm{dd}, J=15.8,9.9 \mathrm{~Hz}, 1 \mathrm{H}) .{ }^{13} \mathrm{C} \mathrm{NMR}$ $\left(101 \mathrm{MHz}, \mathrm{CDCl}_{3}\right) \delta 158.9,157.4,134.2,128.8,114.6,83.6,82.5,63.8,41.1,25.3,25.0$, 15.2. HRMS (ESI $\left.{ }^{+}\right): m / z$ for $\mathrm{C}_{19} \mathrm{H}_{28} \mathrm{~B}_{2} \mathrm{O}_{5} \mathrm{Na}[\mathrm{M}+\mathrm{Na}]^{+}$calcd 381.2021, found 381.2017.

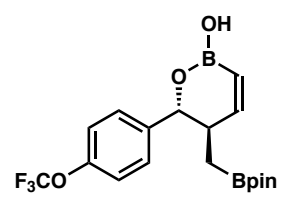

(5R,6R)-5-((4,4,5,5-Tetramethyl-1,3,2-dioxaborolan-2-yl)methyl)-6(4-(trifluoromethoxy)phenyl)-5,6-dihydro-2H-1,2-oxaborinin-2-ol (2e) Prepared according to the general procedure. The crude mixture was purified by flash column chromatography (hexane : ethyl acetate $=$ $5: 1)$ to give the title compound as colorless oil in 90\% yield (36 mg). Enantiomeric excess was determined by HPLC analysis of the corresponding diphenylation product to be $99 \%$ ee $\left(254 \mathrm{~nm}, 25^{\circ} \mathrm{C}\right) ; \mathrm{t}_{1}=7.58 \mathrm{~min}, \mathrm{t}_{2}=8.69 \mathrm{~min}$ [(Chiralpak IA) hexane/i-PrOH, 95:5, $1.0 \mathrm{~mL} / \mathrm{min}] ;[\alpha]_{\mathrm{D}}{ }^{20}=+26.4^{\circ}\left(\mathrm{c} 0.25, \mathrm{CHCl}_{3}\right) ;{ }^{1} \mathrm{H} \mathrm{NMR}\left(400 \mathrm{MHz}, \mathrm{CDCl}_{3}\right) \delta 7.40$ $(\mathrm{d}, J=8.4 \mathrm{~Hz}, 2 \mathrm{H}), 7.20(\mathrm{~d}, J=8.1 \mathrm{~Hz}, 2 \mathrm{H}), 6.81(\mathrm{~d}, J=11.9 \mathrm{~Hz}, 1 \mathrm{H}), 5.75(\mathrm{~d}, J=12.1$ $\mathrm{Hz}, 1 \mathrm{H}), 4.79(\mathrm{~d}, J=9.6 \mathrm{~Hz}, 1 \mathrm{H}), 4.13(\mathrm{~s}, 1 \mathrm{H}), 2.69-2.75(\mathrm{~m}, 1 \mathrm{H}), 1.21(\mathrm{~s}, 12 \mathrm{H}), 0.81$ $(\mathrm{dd}, J=15.9,5.5 \mathrm{~Hz}, 1 \mathrm{H}), 0.73(\mathrm{dd}, J=15.9,9.4 \mathrm{~Hz}, 1 \mathrm{H}) .{ }^{13} \mathrm{C} \mathrm{NMR}\left(151 \mathrm{MHz}, \mathrm{CDCl}_{3}\right)$ 
$\delta 157.2,149.1,140.9,129.0,121.1,120.8$ (q, $J=257 \mathrm{~Hz}), 83.8,82.0,41.1,25.3,25.1$, 14.5. HRMS (ESI $\left.{ }^{+}\right): m / z$ for $\mathrm{C}_{18} \mathrm{H}_{23} \mathrm{~B}_{2} \mathrm{~F}_{3} \mathrm{O}_{5} \mathrm{Na}[\mathrm{M}+\mathrm{Na}]^{+}$calcd 421.1581 , found 421.1568 .

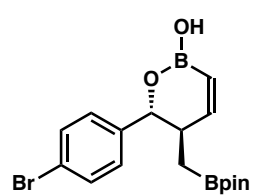

(5R,6R)-6-(4-Bromophenyl)-5-((4,4,5,5-tetramethyl-1,3,2-dioxaborol an-2-yl)methyl)-5,6-dihydro-2H-1,2-oxaborinin-2-ol (2f) Prepared according to the general procedure. The crude mixture was purified by flash column chromatography (hexane : ethyl acetate $=5: 1$ ) to give the title compound as a white solid in $89 \%$ yield $(35 \mathrm{mg})$. Enantiomeric excess was determined by HPLC analysis of the corresponding diphenylation product to be $99 \%$ ee $\left(254 \mathrm{~nm}, 25^{\circ} \mathrm{C}\right) ; \mathrm{t}_{1}=9.96 \mathrm{~min}, \mathrm{t}_{2}=11.3 \mathrm{~min}$ [(Chiralpak IA) hexane/i-PrOH, 95:5, 1.0 $\mathrm{mL} / \mathrm{min}] ;[\alpha]_{\mathrm{D}}{ }^{20}=+28.0^{\circ}\left(\mathrm{c} 0.3, \mathrm{CHCl}_{3}\right) ;{ }^{1} \mathrm{H} \mathrm{NMR}\left(600 \mathrm{MHz}, \mathrm{CDCl}_{3}\right) \delta 7.47(\mathrm{~d}, J=8.3$ $\mathrm{Hz}, 2 \mathrm{H}), 7.24(\mathrm{~d}, J=8.3 \mathrm{~Hz}, 2 \mathrm{H}), 6.80$ (dd, $J=12.1,1.8 \mathrm{~Hz}, 1 \mathrm{H}), 5.74$ (dd, $J=12.1,2.1$ $\mathrm{Hz}, 1 \mathrm{H}), 4.73(\mathrm{~d}, J=9.5 \mathrm{~Hz}, 1 \mathrm{H}), 4.11(\mathrm{~s}, 1 \mathrm{H}), 2.67-2.71(\mathrm{~m}, 1 \mathrm{H}), 1.21(\mathrm{~s}, 12 \mathrm{H}), 0.79$ $(\mathrm{dd}, J=15.9,5.2 \mathrm{~Hz}, 1 \mathrm{H}), 0.72(\mathrm{dd}, J=15.9,9.5 \mathrm{~Hz}, 1 \mathrm{H}) .{ }^{13} \mathrm{C}$ NMR $\left(151 \mathrm{MHz}, \mathrm{CDCl}_{3}\right)$ $\left.\delta 157.1,141.2,131.7,129.3,122.0,83.8,82.1,41.1,25.3,25.1,14.5 . \mathrm{HRMS}_{(\mathrm{ESI}}{ }^{+}\right): \mathrm{m} / z$ for $\mathrm{C}_{17} \mathrm{H}_{23} \mathrm{~B}_{2} \mathrm{BrO}_{4} \mathrm{Na}[\mathrm{M}+\mathrm{Na}]^{+}$calcd 415.0864, found 415.0855 .

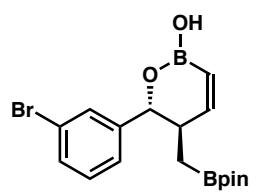

(5R,6R)-6-(3-Bromophenyl)-5-((4,4,5,5-tetramethyl-1,3,2-dioxaborol an-2-yl)methyl)-5,6-dihydro-2H-1,2-oxaborinin-2-ol (2g) Prepared according to the general procedure. The crude mixture was purified by flash column chromatography (hexane : ethyl acetate $=5: 1$ ) to give the title compound as colorless oil in $94 \%$ yield $(37 \mathrm{mg})$. Enantiomeric excess was determined by HPLC analysis of the corresponding diphenylation product to be $99 \%$ ee $\left(254 \mathrm{~nm}, 25^{\circ} \mathrm{C}\right) ; \mathrm{t}_{1}=4.67 \mathrm{~min}, \mathrm{t}_{2}=5.41 \mathrm{~min}$ [(Chiralpak IB) hexane/i-PrOH, 95:5, 1.0 $\mathrm{mL} / \mathrm{min}] ;[\alpha]_{\mathrm{D}}{ }^{20}=+28.9^{\circ}\left(\mathrm{c} 0.9, \mathrm{CHCl}_{3}\right) ;{ }^{1} \mathrm{H} \mathrm{NMR}\left(400 \mathrm{MHz}, \mathrm{CDCl}_{3}\right) \delta 7.53(\mathrm{~s}, 1 \mathrm{H})$, $7.42(\mathrm{~d}, J=7.8 \mathrm{~Hz}, 1 \mathrm{H}), 7.27(\mathrm{~d}, J=8.1 \mathrm{~Hz}, 1 \mathrm{H}), 7.21(\mathrm{dd}, J=7.8,7.7 \mathrm{~Hz}, 1 \mathrm{H}), 6.80(\mathrm{~d}$, $J=11.8 \mathrm{~Hz}, 1 \mathrm{H}), 5.74(\mathrm{dd}, J=12.1,2.1 \mathrm{~Hz}, 1 \mathrm{H}), 4.74(\mathrm{~d}, J=9.4 \mathrm{~Hz}, 1 \mathrm{H}), 4.15(\mathrm{~s}, 1 \mathrm{H})$, $2.67-2.73(\mathrm{~m}, 1 \mathrm{H}), 1.22(\mathrm{~s}, 12 \mathrm{H}), 0.82(\mathrm{dd}, J=16.0,5.5 \mathrm{~Hz}, 1 \mathrm{H}), 0.74(\mathrm{dd}, J=15.9,9.4$ $\mathrm{Hz}, 1 \mathrm{H}) .{ }^{13} \mathrm{C} \mathrm{NMR}\left(101 \mathrm{MHz}, \mathrm{CDCl}_{3}\right) \delta 157.0,144.6,131.2,130.7,130.1,126.3,122.8$, 83.8, 82.0, 41.1, 25.3, 25.1. HRMS $\left(\mathrm{ESI}^{+}\right): m / z$ for $\mathrm{C}_{17} \mathrm{H}_{23} \mathrm{~B}_{2} \mathrm{BrO}_{4} \mathrm{Na}[\mathrm{M}+\mathrm{Na}]^{+}$calcd 415.0864, found 415.0857 .

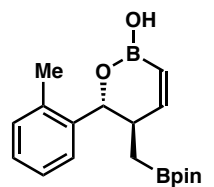

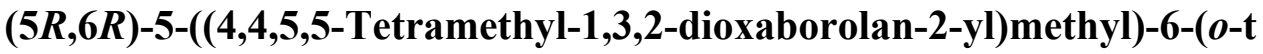
olyl)-5,6-dihydro-2H-1,2-oxaborinin-2-ol (2h) Prepared according to the general procedure. The crude mixture was purified by flash column chromatography (hexane : ethyl acetate $=5: 1$ ) to give the title compound as 
colorless oil in $97 \%$ yield $(32 \mathrm{mg}$ ). Enantiomeric excess was determined by HPLC analysis of the corresponding diphenylation product to be $99 \%$ ee $\left(254 \mathrm{~nm}, 25^{\circ} \mathrm{C}\right) ; \mathrm{t}_{1}=$ $5.00 \mathrm{~min}, \mathrm{t}_{2}=6.31 \mathrm{~min}$ [(Chiralpak IB) hexane $\left./ \mathrm{i}-\mathrm{PrOH}, 95: 5,1.0 \mathrm{~mL} / \mathrm{min}\right] ;[\alpha]_{\mathrm{D}}{ }^{20}=$ $+14.0^{\circ}\left(\mathrm{c} 0.10, \mathrm{CHCl}_{3}\right) ;{ }^{1} \mathrm{H}$ NMR $\left(400 \mathrm{MHz}, \mathrm{CDCl}_{3}\right) \delta 7.39(\mathrm{~d}, J=7.3 \mathrm{~Hz}, 1 \mathrm{H}), 7.14-$ $7.24(\mathrm{~m}, 3 \mathrm{H}), 6.85$ (d, $J=12.0 \mathrm{~Hz}, 1 \mathrm{H}), 5.76(\mathrm{~d}, J=12.0 \mathrm{~Hz}, 1 \mathrm{H}), 5.05(\mathrm{~d}, J=10.2 \mathrm{~Hz}$, $1 \mathrm{H}), 4.08(\mathrm{~s}, 1 \mathrm{H}), 2.85-2.91(\mathrm{~m}, 1 \mathrm{H}), 2.39(\mathrm{~s}, 3 \mathrm{H}), 1.20(\mathrm{~s}, 12 \mathrm{H}), 0.69-0.78(\mathrm{~m}, 2 \mathrm{H})$. ${ }^{13} \mathrm{C}$ NMR $\left(101 \mathrm{MHz}, \mathrm{CDCl}_{3}\right) \delta 157.6,139.9,136.4,130.9,128.0,127.6,126.5,83.7,79.0$, 40.5, 25.3, 25.0, 20.1. HRMS (ESI $\left.{ }^{+}\right): m / z$ for $\mathrm{C}_{18} \mathrm{H}_{26} \mathrm{~B}_{2} \mathrm{O}_{4} \mathrm{Na}[\mathrm{M}+\mathrm{Na}]^{+}$calcd 351.1915, found 351.1902 .

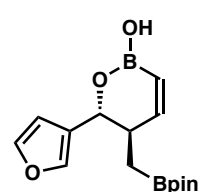

(5R,6R)-6-(Furan-3-yl)-5-((4,4,5,5-tetramethyl-1,3,2-dioxaborolan-2-yl) methyl)-5,6-dihydro-2H-1,2-oxaborinin-2-ol (2i) Prepared according to the general procedure. The crude mixture was purified by flash column chromatography (hexane : ethyl acetate $=5: 1$ ) to give the title compound as colorless oil in 95\% yield $(29 \mathrm{mg})$. Enantiomeric excess was determined by HPLC analysis of the corresponding diphenylation product to be $99 \%$ ee $\left(254 \mathrm{~nm}, 25^{\circ} \mathrm{C}\right) ; \mathrm{t}_{1}=$ $10.7 \mathrm{~min}, \mathrm{t}_{2}=11.4 \mathrm{~min}$ [(Chiralpak IA) hexane/i-PrOH, 95:5, $\left.1.0 \mathrm{~mL} / \mathrm{min}\right] ;[\alpha]_{\mathrm{D}}{ }^{20}=-$ $25.0^{\circ}$ (c $\left.0.20, \mathrm{CHCl}_{3}\right) ;{ }^{1} \mathrm{H} \mathrm{NMR}\left(400 \mathrm{MHz}, \mathrm{CDCl}_{3}\right) \delta 7.39-7.40(\mathrm{~m}, 2 \mathrm{H}), 6.83(\mathrm{~d}, J=$ $12.7 \mathrm{~Hz}, 1 \mathrm{H}), 6.44(\mathrm{~s}, 1 \mathrm{H}), 5.71(\mathrm{~d}, J=12.2 \mathrm{~Hz}, 1 \mathrm{H}), 4.78(\mathrm{~d}, J=8.7 \mathrm{~Hz}, 1 \mathrm{H}), 4.11(\mathrm{~s}$, $1 \mathrm{H}), 2.68-2.73(\mathrm{~m}, 1 \mathrm{H}), 1.23(\mathrm{~s}, 12 \mathrm{H}), 0.92$ (dd, $J=15.8,5.1 \mathrm{~Hz}, 1 \mathrm{H}), 0.77$ (dd, $J=$ $15.8,9.7 \mathrm{~Hz}, 1 \mathrm{H}) .{ }^{13} \mathrm{C} \mathrm{NMR}\left(151 \mathrm{MHz}, \mathrm{CDCl}_{3}\right) \delta 157.1,143.6,140.5,127.1,109.3,83.7$, 74.9, 40.0, 25.3, 25.1, 15.0. HRMS $\left(\mathrm{ESI}^{+}\right): \mathrm{m} / z$ for $\mathrm{C}_{15} \mathrm{H}_{22} \mathrm{~B}_{2} \mathrm{O}_{5} \mathrm{Na}[\mathrm{M}+\mathrm{Na}]^{+}$calcd 327.1551 , found 327.1539 .

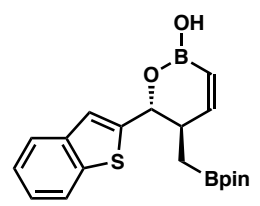

(5R,6R)-6-(Benzo $[b]$ thiophen-2-yl)-5-((4,4,5,5-tetramethyl-1,3,2-diox aborolan-2-yl)methyl)-5,6-dihydro-2H-1,2-oxaborinin-2-ol

Prepared according to the general procedure. The crude mixture was purified by flash column chromatography (hexane : ethyl acetate $=5: 1$ )

to give the title compound as white solid in $95 \%$ yield ( $35 \mathrm{mg}$ ). Enantiomeric excess was determined by HPLC analysis of the corresponding diphenylation product to be $99 \%$ ee $\left(254 \mathrm{~nm}, 25^{\circ} \mathrm{C}\right) ; \mathrm{t}_{1}=16.3 \mathrm{~min}, \mathrm{t}_{2}=18.0 \mathrm{~min}$ [(Chiralpak IA) hexane/i-PrOH, 95:5, 1.0 $\mathrm{mL} / \mathrm{min}] ;[\alpha]_{\mathrm{D}}{ }^{20}=+17.1^{\circ}\left(\mathrm{c} 0.35, \mathrm{CHCl}_{3}\right) ;{ }^{1} \mathrm{H}$ NMR $\left(400 \mathrm{MHz}, \mathrm{CDCl}_{3}\right) \delta 7.80(\mathrm{~d}, J=7.6$ $\mathrm{Hz}, 1 \mathrm{H}), 7.71$ (d, $J=7.2 \mathrm{~Hz}, 1 \mathrm{H}), 7.27-7.35$ (m, 2H), 7.22 (s, 1H), 6.87 (dd, $J=12.1$, $2.3 \mathrm{~Hz}, 1 \mathrm{H}), 5.76(\mathrm{~d}, J=12.2 \mathrm{~Hz}, 1 \mathrm{H}), 5.15(\mathrm{~d}, J=8.2 \mathrm{~Hz}, 1 \mathrm{H}), 4.25(\mathrm{~s}, 1 \mathrm{H}), 2.86-2.91$ (m, 1H), $1.21(\mathrm{~s}, 12 \mathrm{H}), 1.01$ (dd, $J=15.9,5.3 \mathrm{~Hz}, 1 \mathrm{H}), 0.87$ (dd, $J=15.9,9.5 \mathrm{~Hz}, 1 \mathrm{H})$. ${ }^{13} \mathrm{C}$ NMR $\left(151 \mathrm{MHz}, \mathrm{CDCl}_{3}\right) \delta 156.8,146.8,140.0,139.6,124.62,124.58,123.9,122.7$, 
122.4, 83.8, 78.7, 41.3, 25.3, 25.1, 15.1. HRMS (ESI $\left.{ }^{+}\right): m / z$ for $\mathrm{C}_{19} \mathrm{H}_{24} \mathrm{~B}_{2} \mathrm{O}_{4} \mathrm{SNa}[\mathrm{M}+\mathrm{Na}]^{+}$ calcd 393.1479, found 393.1466.

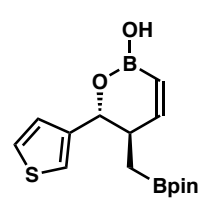

(5R,6R)-5-((4,4,5,5-Tetramethyl-1,3,2-dioxaborolan-2-yl)methyl)-6-(thio phen-3-yl)-5,6-dihydro-2H-1,2-oxaborinin-2-ol (2k) Prepared according to the general procedure. The crude mixture was purified by flash column chromatography (hexane : ethyl acetate $=5: 1$ ) to give the title compound as colorless oil in $94 \%$ yield $(30 \mathrm{mg}$ ). Enantiomeric excess was determined by HPLC analysis of the corresponding diphenylation product to be $99 \%$ ee $\left(254 \mathrm{~nm}, 25^{\circ} \mathrm{C}\right) ; \mathrm{t}_{1}=$ $11.0 \mathrm{~min}, \mathrm{t}_{2}=12.7 \mathrm{~min}$ [(Chiralpak IA) hexane/i-PrOH, 95:5, $\left.1.0 \mathrm{~mL} / \mathrm{min}\right] ;[\alpha]_{\mathrm{D}}{ }^{20}=-5.6^{\circ}$ (c $\left.0.25, \mathrm{CHCl}_{3}\right) ;{ }^{1} \mathrm{H} \mathrm{NMR}\left(400 \mathrm{MHz}, \mathrm{CDCl}_{3}\right) \delta 7.29-7.31(\mathrm{~m}, 1 \mathrm{H}), 7.20-7.21(\mathrm{~m}, 1 \mathrm{H})$, $7.11-7.12(\mathrm{~m}, 1 \mathrm{H}), 6.82(\mathrm{~d}, J=11.9 \mathrm{~Hz}, 1 \mathrm{H}), 5.72(\mathrm{~d}, J=12.3 \mathrm{~Hz}, 1 \mathrm{H}), 4.90$ (d, $J=8.9$ $\mathrm{Hz}, 1 \mathrm{H}), 4.08(\mathrm{~s}, 1 \mathrm{H}), 2.74-2.80(\mathrm{~m}, 1 \mathrm{H}), 1.23(\mathrm{~s}, 12 \mathrm{H}), 0.89(\mathrm{dd}, J=15.9,5.3 \mathrm{~Hz}, 1 \mathrm{H})$, $0.77(\mathrm{dd}, J=15.9,9.7 \mathrm{~Hz}, 1 \mathrm{H}) .{ }^{13} \mathrm{C} \mathrm{NMR}\left(151 \mathrm{MHz}, \mathrm{CDCl}_{3}\right) \delta 157.1,143.7,126.6,126.3$, 122.8, 83.7, 78.5, 40.7, 25.3, 25.1, 15.0. HRMS (ESI $\left.{ }^{+}\right): m / z$ for $\mathrm{C}_{15} \mathrm{H}_{22} \mathrm{~B}_{2} \mathrm{O}_{4} \mathrm{SNa}[\mathrm{M}+\mathrm{Na}]^{+}$ calcd 343.1323, found 343.1337.

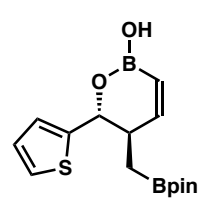

(5R,6R)-5-((4,4,5,5-Tetramethyl-1,3,2-dioxaborolan-2-yl)methyl)-6-(thio phen-2-yl)-5,6-dihydro-2H-1,2-oxaborinin-2-ol (2l) Prepared according to the general procedure. The crude mixture was purified by flash column chromatography (hexane : ethyl acetate $=5: 1$ ) to give the title compound as colorless oil in $91 \%$ yield $(29 \mathrm{mg}$ ). Enantiomeric excess was determined by HPLC analysis of the corresponding diphenylation product to be $99 \%$ ee $\left(254 \mathrm{~nm}, 25^{\circ} \mathrm{C}\right) ; \mathrm{t}_{1}=$ $10.6 \mathrm{~min}, \mathrm{t}_{2}=11.7 \mathrm{~min}$ [(Chiralpak IA) hexane/i-PrOH, 95:5, $\left.1.0 \mathrm{~mL} / \mathrm{min}\right] ;[\alpha]_{\mathrm{D}}{ }^{20}=-$ $16.0^{\circ}\left(\mathrm{c} 0.35, \mathrm{CHCl}_{3}\right) ;{ }^{1} \mathrm{H}$ NMR $\left(400 \mathrm{MHz}, \mathrm{CDCl}_{3}\right) \delta 7.25-7.26(\mathrm{~m}, 1 \mathrm{H}), 7.00-7.01$ (m, $1 \mathrm{H}), 6.94-6.96(\mathrm{~m}, 1 \mathrm{H}), 6.85(\mathrm{~d}, J=11.7 \mathrm{~Hz}, 1 \mathrm{H}), 5.75(\mathrm{~d}, J=12.1 \mathrm{~Hz}, 1 \mathrm{H}), 5.07(\mathrm{~d}, J$ $=8.6 \mathrm{~Hz}, 1 \mathrm{H}), 4.12(\mathrm{~s}, 1 \mathrm{H}), 2.79-2.84(\mathrm{~m}, 1 \mathrm{H}), 1.23(\mathrm{~s}, 12 \mathrm{H}), 0.95(\mathrm{dd}, J=16.0,5.1 \mathrm{~Hz}$, $1 \mathrm{H}), 0.80(\mathrm{dd}, J=15.9,9.7 \mathrm{~Hz}, 1 \mathrm{H}) .{ }^{13} \mathrm{C} \mathrm{NMR}\left(151 \mathrm{MHz}, \mathrm{CDCl}_{3}\right) \delta 156.9,146.1,126.7$, 125.8, 125.4, 122.0, 83.7, 78.1, 41.7, 25.3, 25.1, 15.1. HRMS $\left(\mathrm{ESI}^{+}\right): \mathrm{m} / \mathrm{z}$ for $\mathrm{C}_{15} \mathrm{H}_{22} \mathrm{~B}_{2} \mathrm{O}_{4} \mathrm{SNa}[\mathrm{M}+\mathrm{Na}]^{+}$calcd 343.1323, found 343.1310.

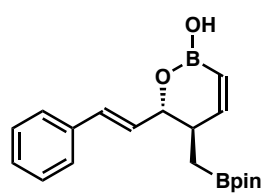

(5R,6S)-6-((E)-Styryl)-5-((4,4,5,5-tetramethyl-1,3,2-dioxaborolan-2yl)methyl)-5,6-dihydro-2H-1,2-oxaborinin-2-ol (2m) Prepared according to the general procedure. The crude mixture was purified by flash column chromatography (hexane : ethyl acetate $=5: 1$ ) to give the title compound as a white solid in $94 \%$ yield $(32 \mathrm{mg})$. Enantiomeric excess was 
determined by HPLC analysis of the corresponding diphenylation product to be $98 \%$ ee $\left(254 \mathrm{~nm}, 25^{\circ} \mathrm{C}\right) ; \mathrm{t}_{1}=13.3 \mathrm{~min}, \mathrm{t}_{2}=14.5 \mathrm{~min}$ [(Chiralpak IA) hexane/i-PrOH, 95:5, 1.0 $\mathrm{mL} / \mathrm{min}] ;[\alpha]_{\mathrm{D}}{ }^{20}=-16.7^{\circ}\left(\mathrm{c} 0.30, \mathrm{CHCl}_{3}\right) ;{ }^{1} \mathrm{H} \mathrm{NMR}\left(400 \mathrm{MHz}, \mathrm{CDCl}_{3}\right) \delta 7.39$ (d, $J=7.4$ $\mathrm{Hz}, 2 \mathrm{H}), 7.31(\mathrm{dd}, J=7.5,7.5 \mathrm{~Hz}, 2 \mathrm{H}), 7.23(\mathrm{dd}, J=7.3,7.3 \mathrm{~Hz}, 1 \mathrm{H}), 6.81(\mathrm{~d}, J=12.0$ $\mathrm{Hz}, 1 \mathrm{H}), 6.60(\mathrm{~d}, J=16.0 \mathrm{~Hz}, 1 \mathrm{H}), 6.26(\mathrm{dd}, J=15.9,7.3 \mathrm{~Hz}, 1 \mathrm{H}), 5.69$ (d, $J=12.1 \mathrm{~Hz}$, 1H), 4.40 (dd, $J=7.8,7.8 \mathrm{~Hz}, 1 \mathrm{H}), 4.08(\mathrm{~s}, 1 \mathrm{H}), 2.55-2.61(\mathrm{~m}, 1 \mathrm{H}), 1.23(\mathrm{~s}, 6 \mathrm{H}), 1.22$ $(\mathrm{s}, 6 \mathrm{H}), 1.05(\mathrm{dd}, J=15.9,5.5 \mathrm{~Hz}, 1 \mathrm{H}), 0.85(\mathrm{dd}, J=15.8,9.6 \mathrm{~Hz}, 1 \mathrm{H}) .{ }^{13} \mathrm{C} \mathrm{NMR}(151$ $\left.\mathrm{MHz} \mathrm{CDCl}_{3}\right) \delta 157.1,136.9,132.8,130.1,128.8,128.1,127.0,121.7,83.7,81.3,39.6$, 25.3, 25.1, 15.1. HRMS (ESI $\left.{ }^{+}\right): m / z$ for $\mathrm{C}_{19} \mathrm{H}_{26} \mathrm{~B}_{2} \mathrm{O}_{4} \mathrm{Na}[\mathrm{M}+\mathrm{Na}]^{+}$calcd 363.1915, found 363.1898 .

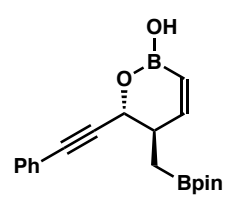

(5R,6R)-6-(Phenylethynyl)-5-((4,4,5,5-tetramethyl-1,3,2-dioxaborolan2-yl)methyl)-5,6-dihydro-2H-1,2-oxaborinin-2-ol

(2n) Prepared according to the general procedure. The crude mixture was purified by flash column chromatography (hexane : ethyl acetate $=5: 1$ ) to give the title compound as colorless oil in $92 \%$ yield $(31 \mathrm{mg})$. Enantiomeric excess was determined by HPLC analysis of the product derived from bromination-oxidation and mono TBS protection to be $99 \%$ ee $\left(254 \mathrm{~nm}, 25^{\circ} \mathrm{C}\right) ; \mathrm{t}_{1}=6.50 \mathrm{~min}, \mathrm{t}_{2}=12.4 \mathrm{~min}$ [(Chiralpak IC) hexane/i-PrOH, 98:2, $1.0 \mathrm{~mL} / \mathrm{min}] ;[\alpha]_{\mathrm{D}}{ }^{20}=-8.0^{\circ}$ (c $\left.0.70, \mathrm{CHCl}_{3}\right) ;{ }^{1} \mathrm{H}$ NMR (400 MHz, $\left.\mathrm{CDCl}_{3}\right) \delta 7.44-7.46(\mathrm{~m}, 2 \mathrm{H}), 7.28-7.31(\mathrm{~m}, 3 \mathrm{H}), 6.86(\mathrm{dd}, J=12.1$, $2.1 \mathrm{~Hz}, 1 \mathrm{H}), 5.72(\mathrm{~d}, J=12.1 \mathrm{~Hz}, 1 \mathrm{H}), 4.77(\mathrm{~d}, J=8.7 \mathrm{~Hz}, 1 \mathrm{H}), 4.13(\mathrm{~s}, 1 \mathrm{H}), 2.80-2.85$ $(\mathrm{m}, 1 \mathrm{H}), 1.26(\mathrm{~s}, 12 \mathrm{H}), 1.23-1.30(\mathrm{~m}, 1 \mathrm{H}), 0.92(\mathrm{dd}, J=16.0,10.4 \mathrm{~Hz}, 1 \mathrm{H}) .{ }^{13} \mathrm{C} \mathrm{NMR}$ $\left(151 \mathrm{MHz}, \mathrm{CDCl}_{3}\right) \delta 156.6,132.2,128.8,128.5,122.9,88.6,85.2,83.8,71.0,40.6,25.3$, 25.1. HRMS (ESI $\left.{ }^{+}\right): m / z$ for $\mathrm{C}_{19} \mathrm{H}_{24} \mathrm{~B}_{2} \mathrm{O}_{4} \mathrm{Na}[\mathrm{M}+\mathrm{Na}]^{+}$calcd 361.1758 , found 361.1771 .

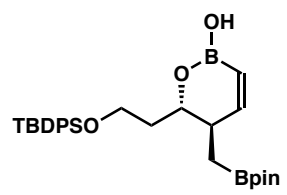

$(5 R, 6 S)-6-(2-(($ tert-Butyldiphenylsilyl)oxy)ethyl)-5-((4,4,5,5-tetrame thyl-1,3,2-dioxaborolan-2-yl)methyl)-5,6-dihydro-2H-1,2-oxaborini n-2-ol (2o) Prepared according to the general procedure. The crude mixture was purified by flash column chromatography (hexane : ethyl acetate $=7.5: 1)$ to give the title compound as colorless oil in $96 \%$ yield $(50 \mathrm{mg})$. Enantiomeric excess was determined by HPLC analysis of the corresponding diphenylation product to be $95 \%$ ee $\left(254 \mathrm{~nm}, 25^{\circ} \mathrm{C}\right) ; \mathrm{t}_{1}=6.51 \mathrm{~min}, \mathrm{t}_{2}=7.32 \mathrm{~min}$ [(Chiralpak IA) hexane/i-PrOH, 98:2, $1.0 \mathrm{~mL} / \mathrm{min}] ;[\alpha]_{\mathrm{D}}{ }^{20}=-50.4^{\circ}\left(\mathrm{c} 0.25, \mathrm{CHCl}_{3}\right) ;{ }^{1} \mathrm{H}$ NMR (400 MHz, $\left.\mathrm{CDCl}_{3}\right) \delta 7.67(\mathrm{dd}, J=6.5,6.5 \mathrm{~Hz}, 4 \mathrm{H}), 7.36-7.44(\mathrm{~m}, 6 \mathrm{H}), 6.77$ (dd, $J=12.1,2.8 \mathrm{~Hz}, 1 \mathrm{H}), 5.59(\mathrm{~d}, J=12.0 \mathrm{~Hz}, 1 \mathrm{H}), 3.97-4.02(\mathrm{~m}, 1 \mathrm{H}), 3.77-3.88(\mathrm{~m}, 2 \mathrm{H})$, $3.76(\mathrm{~s}, 1 \mathrm{H}), 2.40-2.41(\mathrm{~m}, 1 \mathrm{H}), 1.85-1.93(\mathrm{~m}, 1 \mathrm{H}), 1.70-1.78(\mathrm{~m}, 1 \mathrm{H}), 1.24(\mathrm{~s}, 12 \mathrm{H})$, 
$1.04(\mathrm{~s}, 9 \mathrm{H}), 0.99$ (dd, $J=15.8,5.6 \mathrm{~Hz}, 1 \mathrm{H}), 0.84(\mathrm{dd}, J=15.8,9.5 \mathrm{~Hz}, 1 \mathrm{H}) .{ }^{13} \mathrm{C} \mathrm{NMR}$ $\left(101 \mathrm{MHz}, \mathrm{CDCl}_{3}\right) \delta 157.1,136.0,135.9,134.4,134.3,129.9$ (two overlapping $\mathrm{C}$ signals), 127.93, 127.91, 83.7, 76.5, 60.8, 39.1, 38.4, 27.2, 25.2, 25.1, 19.5. HRMS (ESI $\left.{ }^{+}\right): m / z$ for $\mathrm{C}_{29} \mathrm{H}_{42} \mathrm{~B}_{2} \mathrm{O}_{5} \mathrm{SiNa}[\mathrm{M}+\mathrm{Na}]^{+}$calcd 543.2885, found 543.2904.

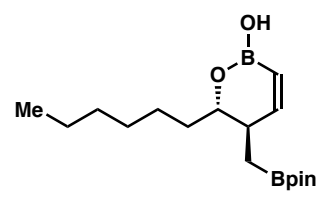

$(5 R, 6 S)-6-H e x y l-5-((4,4,5,5-t e t r a m e t h y l-1,3,2-d i o x a b o r o l a n-2-y l)$ methyl)-5,6-dihydro-2H-1,2-oxaborinin-2-ol (2p) Prepared according to the general procedure. The crude mixture was purified by flash column chromatography (hexane : ethyl acetate $=7.5: 1$ ) to give the title compound as colorless oil in 93\% yield $(30 \mathrm{mg})$. Enantiomeric excess was determined by HPLC analysis of the diphenylation product to be $95 \%$ ee $\left(254 \mathrm{~nm}, 25{ }^{\circ} \mathrm{C}\right)$; $\mathrm{t}_{1}=10.5 \mathrm{~min}, \mathrm{t}_{2}=11.2 \mathrm{~min}\left[(\right.$ Chiralpak IA) hexane $/ \mathrm{i}-\mathrm{PrOH}, 98: 2,1.0 \mathrm{~mL} / \mathrm{min}] ;[\alpha]_{\mathrm{D}}{ }^{20}=$ $-65.3^{\circ}$ (c 0.15, $\left.\mathrm{CHCl}_{3}\right) ;{ }^{1} \mathrm{H} \mathrm{NMR}\left(400 \mathrm{MHz}, \mathrm{CDCl}_{3}\right) \delta 6.77(\mathrm{dd}, J=12.1,2.9 \mathrm{~Hz}, 1 \mathrm{H})$, $5.60(\mathrm{~d}, J=12.1 \mathrm{~Hz}, 1 \mathrm{H}), 3.89(\mathrm{~s}, 1 \mathrm{H}), 3.78(\mathrm{ddd}, J=6.2,6.2,6.2 \mathrm{~Hz}, 1 \mathrm{H}), 2.37-2.43$ (m, 1H), $1.44-1.58(\mathrm{~m}, 2 \mathrm{H}), 1.26-1.29(\mathrm{~m}, 20 \mathrm{H}), 1.25$ (s, 12H), 0.98 (dd, $J=16.0,5.9$ $\mathrm{Hz}, 1 \mathrm{H}), 0.82-0.90(\mathrm{~m}, 4 \mathrm{H}) .{ }^{13} \mathrm{C} \mathrm{NMR}\left(151 \mathrm{MHz}, \mathrm{CDCl}_{3}\right) \delta 157.3,83.7,79.8,38.8$, 35.5, 32.2, 29.6, 25.8, 25.2, 25.1, 23.0, 14.5. HRMS $\left(\mathrm{ESI}^{+}\right): \mathrm{m} / z$ for $\mathrm{C}_{17} \mathrm{H}_{32} \mathrm{~B}_{2} \mathrm{O}_{4} \mathrm{Na}$ $[\mathrm{M}+\mathrm{Na}]^{+}$calcd 345.2384 , found 345.2385 .

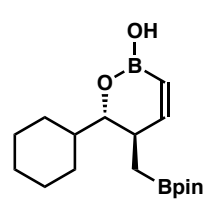

(5R,6S)-6-Cyclohexyl-5-((4,4,5,5-tetramethyl-1,3,2-dioxaborolan-2-yl)m ethyl)-5,6-dihydro-2H-1,2-oxaborinin-2-ol (2q) Prepared according to the general procedure. The crude mixture was purified by flash column chromatography (hexane : ethyl acetate $=7.5: 1$ ) to give the title compound as colorless oil in $88 \%$ yield $(28 \mathrm{mg})$. Enantiomeric excess was determined by HPLC analysis of the corresponding diphenylation product to be $99 \%$ ee $\left(254 \mathrm{~nm}, 25^{\circ} \mathrm{C}\right) ; \mathrm{t}_{1}=$ $7.67 \mathrm{~min}, \mathrm{t}_{2}=8.90 \mathrm{~min}$ [(Chiralpak IA) hexane/i-PrOH, 95:5, $\left.1.0 \mathrm{~mL} / \mathrm{min}\right] ;[\alpha]_{\mathrm{D}}{ }^{20}=-$ 98.5 (c 0.40, $\left.\mathrm{CHCl}_{3}\right) ;{ }^{1} \mathrm{H}$ NMR $\left(400 \mathrm{MHz}, \mathrm{CDCl}_{3}\right) \delta 6.80(\mathrm{dd}, J=12.2,3.6 \mathrm{~Hz}, 1 \mathrm{H})$, $5.58(\mathrm{~d}, J=12.3 \mathrm{~Hz}, 1 \mathrm{H}), 3.91(\mathrm{~s}, 1 \mathrm{H}), 3.59(\mathrm{dd}, J=5.4,5.4 \mathrm{~Hz}, 1 \mathrm{H}), 2.54-2.60(\mathrm{~m}$, $1 \mathrm{H}), 1.74-1.76(\mathrm{~m}, 3 \mathrm{H}), 1.63-1.65(\mathrm{~m}, 1 \mathrm{H}), 1.47-1.52(\mathrm{~m}, 1 \mathrm{H}), 1.25(\mathrm{~s}, 12 \mathrm{H}), 1.06-$ $1.27(\mathrm{~m}, 6 \mathrm{H}), 0.97(\mathrm{dd}, J=15.6,6.3 \mathrm{~Hz}, 1 \mathrm{H}), 0.87(\mathrm{dd}, J=15.7,8.7 \mathrm{~Hz}, 1 \mathrm{H}) .{ }^{13} \mathrm{C} \mathrm{NMR}$ $\left(151 \mathrm{MHz} \mathrm{CDCl}_{3}\right) \delta 157.4,84.0,83.6,41.3,35.6,30.7,27.7,26.83,26.76,26.55,25.3$, 25.2. HRMS (ESI ${ }^{+}$): $m / z$ for $\mathrm{C}_{17} \mathrm{H}_{30} \mathrm{~B}_{2} \mathrm{O}_{4} \mathrm{Na}[\mathrm{M}+\mathrm{Na}]^{+}$calcd 343.2228, found 343.2225.

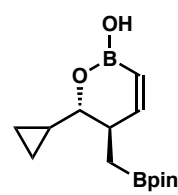

(5R,6S)-6-Cyclopropyl-5-((4,4,5,5-tetramethyl-1,3,2-dioxaborolan-2-yl)m ethyl)-5,6-dihydro-2H-1,2-oxaborinin-2-ol (2r) Prepared according to the general procedure. The crude mixture was purified by flash column 
chromatography (hexane : ethyl acetate $=5: 1$ ) to give the title compound as colorless oil in $90 \%$ yield $(25 \mathrm{mg})$. Enantiomeric excess was determined by HPLC analysis of the corresponding diphenylation product to be $93 \%$ ee $\left(254 \mathrm{~nm}, 25{ }^{\circ} \mathrm{C}\right) ; \mathrm{t}_{1}=9.96 \mathrm{~min}, \mathrm{t}_{2}=$ $11.0 \mathrm{~min}$ [(Chiralpak IC) hexane/i-PrOH, 98:2, $1.0 \mathrm{~mL} / \mathrm{min}] ;[\alpha]_{\mathrm{D}}{ }^{20}=-80.9^{\circ}$ (c 0.85 , $\left.\mathrm{CHCl}_{3}\right) ;{ }^{1} \mathrm{H} \mathrm{NMR}\left(400 \mathrm{MHz}, \mathrm{CDCl}_{3}\right) \delta 6.81(\mathrm{dd}, J=12.0,2.2 \mathrm{~Hz}, 1 \mathrm{H}), 5.64(\mathrm{~d}, J=12.0$ $\mathrm{Hz}, 1 \mathrm{H}), 4.06(\mathrm{~s}, 1 \mathrm{H}), 3.06(\mathrm{dd}, J=7.7,7.7 \mathrm{~Hz}, 1 \mathrm{H}), 2.61-2.67(\mathrm{~m}, 1 \mathrm{H}), 1.25(\mathrm{~s}, 12 \mathrm{H})$, $1.14(\mathrm{dd}, J=15.6,5.8 \mathrm{~Hz}, 1 \mathrm{H}), 1.01-1.09$ (m 1H), 0.89 (dd, $J=15.8,9.2 \mathrm{~Hz}, 1 \mathrm{H}), 0.49$ $-0.57(\mathrm{~m}, 2 \mathrm{H}), 0.37-0.39(\mathrm{~m}, 1 \mathrm{H}), 0.29-0.33(\mathrm{~m}, 1 \mathrm{H}) .{ }^{13} \mathrm{C} \mathrm{NMR}\left(101 \mathrm{MHz}, \mathrm{CDCl}_{3}\right) \delta$ 157.3, 84.8, 83.6, 40.3, 25.2, 25.1, 16.3, 4.1, 3.0. HRMS (ESI $\left.{ }^{+}\right): m / z$ for $\mathrm{C}_{14} \mathrm{H}_{24} \mathrm{~B}_{2} \mathrm{O}_{4} \mathrm{Na}$ $[\mathrm{M}+\mathrm{Na}]^{+}$calcd 301.1758, found 301.1758.

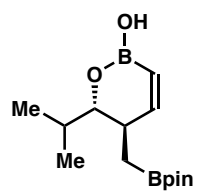

$(5 R, 6 S)-6$-Isopropyl-5-((4,4,5,5-tetramethyl-1,3,2-dioxaborolan-2-yl)met hyl)-5,6-dihydro-2H-1,2-oxaborinin-2-ol (2s) Prepared according to the general procedure. The crude mixture was purified by flash column chromatography (hexane : ethyl acetate $=7.5: 1$ ) to give the title compound as colorless oil in $86 \%$ yield $(24 \mathrm{mg})$. Enantiomeric excess was determined by HPLC analysis of the corresponding diphenylation product to be $98 \%$ ee $\left(254 \mathrm{~nm}, 25^{\circ} \mathrm{C}\right) ; \mathrm{t}_{1}=$ $7.40 \mathrm{~min}, \mathrm{t}_{2}=7.85 \mathrm{~min}$ [(Chiralpak IC) hexane/i-PrOH, 98:2, $\left.1.0 \mathrm{~mL} / \mathrm{min}\right] ;[\alpha]_{\mathrm{D}}{ }^{20}=-$ $72.4^{\circ}\left(\mathrm{c} 0.55, \mathrm{CHCl}_{3}\right) ;{ }^{1} \mathrm{H} \mathrm{NMR}\left(400 \mathrm{MHz}, \mathrm{CDCl}_{3}\right) \delta 6.78(\mathrm{dd}, J=12.0,2.9 \mathrm{~Hz}, 1 \mathrm{H})$, $5.59(\mathrm{~d}, J=12.1 \mathrm{~Hz}, 1 \mathrm{H}), 3.88(\mathrm{~s}, 1 \mathrm{H}), 3.58(\mathrm{dd}, J=6.0,5.3 \mathrm{~Hz}, 1 \mathrm{H}), 2.52-2.58(\mathrm{~m}$, $1 \mathrm{H}), 1.81-1.89(\mathrm{~m}, 1 \mathrm{H}), 1.25(\mathrm{~s}, 12 \mathrm{H}), 0.94-0.99(\mathrm{~m}, 4 \mathrm{H}), 0.90(\mathrm{~d}, J=6.6 \mathrm{~Hz}, 3 \mathrm{H})$, $0.86(\mathrm{dd}, J=15.7,8.7 \mathrm{~Hz}, 1 \mathrm{H}) .{ }^{13} \mathrm{C} \mathrm{NMR}\left(151 \mathrm{MHz}, \mathrm{CDCl}_{3}\right) \delta 157.6,84.4,83.6,36.2$, 31.1, 25.2, 25.1, 20.5, 17.2. HRMS $\left(\mathrm{ESI}^{+}\right): \mathrm{m} / z$ for $\mathrm{C}_{14} \mathrm{H}_{26} \mathrm{~B}_{2} \mathrm{O}_{4} \mathrm{Na}[\mathrm{M}+\mathrm{Na}]^{+}$calcd 303.1915, found 303.1921.

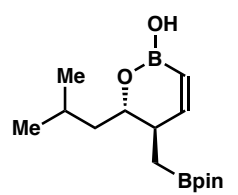

(5R,6S)-6-Isobutyl-5-((4,4,5,5-tetramethyl-1,3,2-dioxaborolan-2-yl)met hyl)-5,6-dihydro-2H-1,2-oxaborinin-2-ol (2t) Prepared according to the general procedure. The crude mixture was purified by flash column chromatography (hexane : ethyl acetate $=7.5: 1$ ) to give the title compound as colorless oil in $92 \%$ yield $(27 \mathrm{mg})$. Enantiomeric excess was determined by HPLC analysis of the corresponding diphenylation product to be $96 \%$ ee $\left(254 \mathrm{~nm}, 25^{\circ} \mathrm{C}\right) ; \mathrm{t}_{1}=$ $11.3 \mathrm{~min}, \mathrm{t}_{2}=11.8 \mathrm{~min}$ [(Chiralpak IA) hexane/i-PrOH, 98:2, $\left.1.0 \mathrm{~mL} / \mathrm{min}\right] ;[\alpha]_{\mathrm{D}}{ }^{20}=-$ $84.0^{\circ}$ (c 0.20, $\left.\mathrm{CHCl}_{3}\right) ;{ }^{1} \mathrm{H}$ NMR $\left(400 \mathrm{MHz}, \mathrm{CDCl}_{3}\right) \delta 6.78(\mathrm{dd}, J=11.9,3.4 \mathrm{~Hz}, 1 \mathrm{H})$, $5.60(\mathrm{~d}, J=12.0 \mathrm{~Hz}, 1 \mathrm{H}), 3.86-3.90(\mathrm{~m}, 2 \mathrm{H}), 2.32-2.38(\mathrm{~m}, 1 \mathrm{H}), 1.82-1.91(\mathrm{~m}, 1 \mathrm{H})$, $1.52-1.59(\mathrm{~m}, 1 \mathrm{H}), 1.26-1.32(\mathrm{~m}, 1 \mathrm{H}), 1.25(\mathrm{~s}, 12 \mathrm{H}), 0.98(\mathrm{dd}, J=15.8,6.3 \mathrm{~Hz}, 1 \mathrm{H})$, $0.92(\mathrm{~d}, J=7.0 \mathrm{~Hz}, 3 \mathrm{H}), 0.89(\mathrm{~d}, J=6.8 \mathrm{~Hz}, 3 \mathrm{H}), 0.86(\mathrm{dd}, J=15.8,8.7 \mathrm{~Hz}, 1 \mathrm{H}) .{ }^{13} \mathrm{C}$ 
NMR (151 MHz, $\left.\mathrm{CDCl}_{3}\right) \delta 157.3,83.7,77.7,44.9,39.4,25.2,25.1,24.6,24.0,22.0$. HRMS (ESI ${ }^{+}$): $m / z$ for $\mathrm{C}_{15} \mathrm{H}_{28} \mathrm{~B}_{2} \mathrm{O}_{4} \mathrm{Na}[\mathrm{M}+\mathrm{Na}]^{+}$calcd 317.2071, found 317.2069.

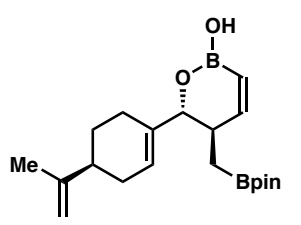

(5R,6R)-6-((S)-4-(Prop-1-en-2-yl)cyclohex-1-en-1-yl)-5-((4,4,5,5-tet ramethyl-1,3,2-dioxaborolan-2-yl)methyl)-5,6-dihydro-2H-1,2-oxa borinin-2-ol (8) Prepared according to the general procedure. The crude mixture was purified by flash column chromatography (hexane : ethyl acetate $=5: 1)$ to give the title compound as colorless oil in $89 \%$ yield (32 $\mathrm{mg})$. The $d r$ and $Z / E$ selectivity were determined by ${ }^{1} \mathrm{H}$ NMR analysis to be $>50: 1 .[\alpha]_{\mathrm{D}}{ }^{20}=-79.1^{\circ}$ (c $\left.0.70, \mathrm{CHCl}_{3}\right) ;{ }^{1} \mathrm{H}$ NMR $\left(600 \mathrm{MHz}, \mathrm{CDCl}_{3}\right) \delta 6.79$ (dd, $\left.J=12.1,1.4 \mathrm{~Hz}, 1 \mathrm{H}\right), 5.72$ (s, $1 \mathrm{H}), 5.65(\mathrm{dd}, J=12.1,2.4 \mathrm{~Hz}, 1 \mathrm{H}), 4.71(\mathrm{~s}, 2 \mathrm{H}), 4.10$ (d, $J=10.4 \mathrm{~Hz}, 1 \mathrm{H}), 4.04(\mathrm{~s}, 1 \mathrm{H})$, $2.58-2.62(\mathrm{~m}, 1 \mathrm{H}), 2.29-2.33(\mathrm{~m}, 1 \mathrm{H}), 2.13-2.16(\mathrm{~m}, 2 \mathrm{H}), 1.86-2.03(\mathrm{~m}, 3 \mathrm{H}), 1.73$ $(\mathrm{s}, 3 \mathrm{H}), 1.47-1.54(\mathrm{~m}, 1 \mathrm{H}), 1.253(\mathrm{~s}, 6 \mathrm{H}), 1.249(\mathrm{~s}, 6 \mathrm{H}), 0.90(\mathrm{dd}, J=16.0,4.2 \mathrm{~Hz}, 1 \mathrm{H})$, $0.65(\mathrm{dd}, J=15.9,10.8 \mathrm{~Hz}, 1 \mathrm{H}) .{ }^{13} \mathrm{C} \mathrm{NMR}\left(101 \mathrm{MHz}, \mathrm{CDCl}_{3}\right) \delta 157.8,150.2,136.8$, 126.9, 109.0, 85.3, 83.7, 41.8, 36.4, 31.0, 27.9, 25.3, 25.1, 24.1, 21.0. HRMS (ESI $\left.{ }^{+}\right): m / z$ for $\mathrm{C}_{20} \mathrm{H}_{32} \mathrm{~B}_{2} \mathrm{O}_{4} \mathrm{Na}[\mathrm{M}+\mathrm{Na}]^{+}$calcd 381.2384, found 381.2377.

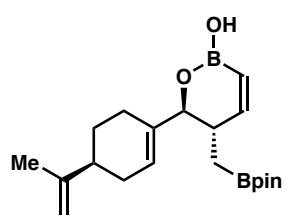

(5S,6S)-6-((S)-4-(Prop-1-en-2-yl)cyclohex-1-en-1-yl)-5-((4,4,5,5-tetr amethyl-1,3,2-dioxaborolan-2-yl)methyl)-5,6-dihydro-2H-1,2-oxab orinin-2-ol (9) Prepared according to the general procedure. The crude mixture was purified by flash column chromatography (hexane : ethyl acetate $=5: 1)$ to give the title compound as colorless oil in $84 \%$ yield (30 mg). The $d r$ and $Z / E$ selectivity were determined by ${ }^{1} \mathrm{H}$ NMR analysis to be $>50: 1$. $[\alpha]_{\mathrm{D}}{ }^{20}=-15.1^{\circ}$ (c 0.70, $\mathrm{CHCl}_{3}$ ); ${ }^{1} \mathrm{H}$ NMR (600 MHz, $\left.\mathrm{CDCl}_{3}\right) \delta 6.79(\mathrm{dd}, J=12.1,2.0 \mathrm{~Hz}, 1 \mathrm{H}), 5.69(\mathrm{~s}, 1 \mathrm{H})$, $5.64(\mathrm{dd}, J=12.1,2.1 \mathrm{~Hz}, 1 \mathrm{H}), 4.73(\mathrm{~s}, 1 \mathrm{H}), 4.70$ (s, 1H), $4.13(\mathrm{~d}, J=9.5 \mathrm{~Hz}, 1 \mathrm{H}), 4.08$ $(\mathrm{s}, 1 \mathrm{H}), 2.60-2.64(\mathrm{~m}, 1 \mathrm{H}), 2.13-2.21(\mathrm{~m}, 3 \mathrm{H}), 1.96-2.04(\mathrm{~m}, 2 \mathrm{H}), 1.84-1.86(\mathrm{~m}$, $1 \mathrm{H}), 1.73(\mathrm{~s}, 3 \mathrm{H}), 1.46-1.53(\mathrm{~m}, 1 \mathrm{H}), 1.25(\mathrm{~s}, 12 \mathrm{H}), 0.89$ (dd, $J=16.1,4.4 \mathrm{~Hz}, 1 \mathrm{H})$, $0.68(\mathrm{dd}, J=16.0,10.3 \mathrm{~Hz}, 1 \mathrm{H}) .{ }^{13} \mathrm{C} \mathrm{NMR}\left(101 \mathrm{MHz}, \mathrm{CDCl}_{3}\right) \delta 157.6,149.9,136.9$, 126.2, 109.0, 85.2, 83.6, 41.1, 36.6, 30.7, 27.6, 25.3, 25.1, 24.1, 21.2. HRMS (ESI $\left.{ }^{+}\right): m / z$ for $\mathrm{C}_{20} \mathrm{H}_{32} \mathrm{~B}_{2} \mathrm{O}_{4} \mathrm{Na}[\mathrm{M}+\mathrm{Na}]^{+}$calcd 381.2384, found 381.2385.

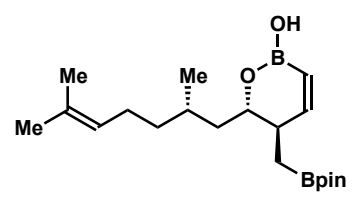

(5R,6S)-6-((S)-2,6-Dimethylhept-5-en-1-yl)-5-((4,4,5,5-tetramet hyl-1,3,2-dioxaborolan-2-yl)methyl)-5,6-dihydro-2H-1,2-oxabor inin-2-ol (11) Prepared according to the general procedure. The crude mixture was purified by flash column chromatography (hexane : ethyl acetate $=7.5: 1$ ) to give the title compound as colorless oil in $91 \%$ yield 
(33 mg). The $d r$ and $Z / E$ selectivity were determined by ${ }^{1} \mathrm{H}$ NMR analysis to be $>50: 1$. $[\alpha]_{\mathrm{D}}{ }^{20}=-89.5^{\circ}\left(\mathrm{c} 0.55, \mathrm{CHCl}_{3}\right) ;{ }^{1} \mathrm{H} \mathrm{NMR}\left(600 \mathrm{MHz}, \mathrm{CDCl}_{3}\right) \delta 6.78(\mathrm{dd}, J=12.1,3.6 \mathrm{~Hz}$, $1 \mathrm{H}), 5.60(\mathrm{dd}, J=12.1,0.9 \mathrm{~Hz}, 1 \mathrm{H}), 5.09(\mathrm{t}, J=6.9 \mathrm{~Hz}, 1 \mathrm{H}), 3.89-3.92(\mathrm{~m}, 1 \mathrm{H}), 3.87(\mathrm{~s}$, $1 \mathrm{H}), 2.33-2.37(\mathrm{~m}, 1 \mathrm{H}), 1.98-2.04(\mathrm{~m}, 1 \mathrm{H}), 1.88-1.94(\mathrm{~m}, 1 \mathrm{H}), 1.68-1.71(\mathrm{~m}, 4 \mathrm{H})$, $1.60(\mathrm{~s}, 3 \mathrm{H}), 1.39-1.50(\mathrm{~m}, 3 \mathrm{H}), 1.25(\mathrm{~s}, 12 \mathrm{H}), 1.06-1.12(\mathrm{~m}, 1 \mathrm{H}), 0.98$ (dd, $J=15.8$, $6.3 \mathrm{~Hz}, 1 \mathrm{H}), 0.92(\mathrm{~d}, J=6.7 \mathrm{~Hz}, 3 \mathrm{H}), 0.87(\mathrm{dd}, J=15.8,8.6 \mathrm{~Hz}, 1 \mathrm{H}) .{ }^{13} \mathrm{C}$ NMR $(101$ $\left.\mathrm{MHz}, \mathrm{CDCl}_{3}\right) \delta 156.9,131.4,125.3,83.6,77.8,43.3,39.0,36.6,29.5,26.1,25.7,25.2$, 25.1, 20.7, 18.0. HRMS $\left(\mathrm{ESI}^{+}\right): \mathrm{m} / z$ for $\mathrm{C}_{20} \mathrm{H}_{36} \mathrm{~B}_{2} \mathrm{O}_{4} \mathrm{Na}[\mathrm{M}+\mathrm{Na}]^{+}$calcd 385.2697, found 385.2698 .

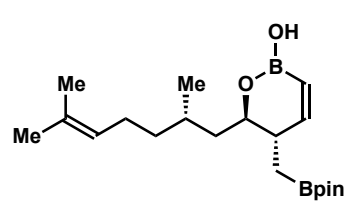

(5S,6R)-6-((S)-2,6-Dimethylhept-5-en-1-yl)-5-((4,4,5,5-tetramet hyl-1,3,2-dioxaborolan-2-yl)methyl)-5,6-dihydro-2H-1,2-oxabor

inin-2-ol (12) Prepared according to the general procedure. The crude mixture was purified by flash column chromatography (hexane : ethyl acetate $=7.5: 1$ ) to give the title compound as colorless oil in $94 \%$ yield (34 mg). The $d r$ and $Z / E$ selectivity were determined by ${ }^{1} \mathrm{H}$ NMR analysis to be $>50: 1$. $[\alpha]_{\mathrm{D}}^{20}=+62.6^{\circ}\left(\mathrm{c} 1.00, \mathrm{CHCl}_{3}\right) ;{ }^{1} \mathrm{H} \mathrm{NMR}\left(600 \mathrm{MHz}, \mathrm{CDCl}_{3}\right) \delta 6.78(\mathrm{dd}, J=12.1,3.3 \mathrm{~Hz}$, $1 \mathrm{H}), 5.60(\mathrm{dd}, J=12.1,1.1 \mathrm{~Hz}, 1 \mathrm{H}), 5.09(\mathrm{t}, J=6.9 \mathrm{~Hz}, 1 \mathrm{H}), 3.88-3.91(\mathrm{~m}, 2 \mathrm{H}), 2.32-$ $2.36(\mathrm{~m}, 1 \mathrm{H}), 1.92-2.03(\mathrm{~m}, 2 \mathrm{H}), 1.72-1.78(\mathrm{~m}, 1 \mathrm{H}), 1.68(\mathrm{~s}, 3 \mathrm{H}), 1.60(\mathrm{~s}, 3 \mathrm{H}), 1.17-$ $1.32(\mathrm{~m}, 16 \mathrm{H}), 0.98(\mathrm{dd}, J=15.8,6.1 \mathrm{~Hz}, 1 \mathrm{H}), 0.89$ (d, $J=6.5 \mathrm{~Hz}, 3 \mathrm{H}), 0.85$ (dd, $J=$ $15.8,8.8 \mathrm{~Hz}, 1 \mathrm{H}) .{ }^{13} \mathrm{C} \mathrm{NMR}\left(101 \mathrm{MHz}, \mathrm{CDCl}_{3}\right) \delta 157.3,131.4,125.2,121.0,83.7,77.4$, 43.1, 39.6, 38.4, 28.9, 26.1, 25.9, 25.2, 25.1, 19.2, 18.0. HRMS $\left(\mathrm{ESI}^{+}\right): \mathrm{m} / \mathrm{z}$ for $\mathrm{C}_{20} \mathrm{H}_{36} \mathrm{~B}_{2} \mathrm{O}_{4} \mathrm{Na}[\mathrm{M}+\mathrm{Na}]^{+}$calcd 385.2697, found 385.2694.

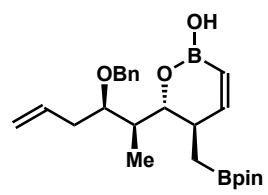

(5R,6R)-6-((2R,3R)-3-(Benzyloxy)hex-5-en-2-yl)-5-((4,4,5,5-tetramet hyl-1,3,2-dioxaborolan-2-yl)methyl)-5,6-dihydro-2H-1,2-oxaborinin -2-ol (14) Prepared according to the general procedure. The crude mixture was purified by flash column chromatography (hexane : ethyl acetate $=7.5: 1)$ to give the title compound as colorless oil in $61 \%$ yield $(26 \mathrm{mg})$. The $Z / E$ selectivity $(>50: 1)$ and $d r(>30: 1)$ were determined by ${ }^{1} \mathrm{H}$ NMR analysis. $[\alpha]_{\mathrm{D}}{ }^{20}=-21.3^{\circ}$ (c $\left.0.60, \mathrm{CHCl}_{3}\right) ;{ }^{1} \mathrm{H} \mathrm{NMR}\left(600 \mathrm{MHz}, \mathrm{CDCl}_{3}\right) \delta 7.30-7.36(\mathrm{~m}, 4 \mathrm{H}), 7.24-7.26(\mathrm{~m}, 1 \mathrm{H})$, $6.86(\mathrm{dd}, J=12.0,5.6 \mathrm{~Hz}, 1 \mathrm{H}), 5.73(\mathrm{ddt}, J=17.4,10.0,7.3 \mathrm{~Hz}, 1 \mathrm{H}), 5.58(\mathrm{~d}, J=12.1$ $\mathrm{Hz}, 1 \mathrm{H}), 5.06(\mathrm{dd}, J=17.1,0.8 \mathrm{~Hz}, 1 \mathrm{H}), 5.01(\mathrm{~d}, J=10.1 \mathrm{~Hz}, 1 \mathrm{H}), 4.64\left(\mathrm{~d}, J_{A B}=11.5 \mathrm{~Hz}\right.$, $1 \mathrm{H}), 4.57\left(\mathrm{~d}, J_{A B}=11.5 \mathrm{~Hz}, 1 \mathrm{H}\right), 3.91(\mathrm{~d}, J=9.5 \mathrm{~Hz}, 1 \mathrm{H}), 3.85-3.88(\mathrm{~m}, 1 \mathrm{H}), 3.84(\mathrm{~s}$, 1H), 2.61 (app. dt, $J=7.1,6.7 \mathrm{~Hz}, 1 \mathrm{H}$ ), 2.50 (ddd, $J=14.0,6.4,6.4 \mathrm{~Hz}, 1 \mathrm{H}$ ), 2.17 (ddd, $J$ $=14.0,7.7,7.7 \mathrm{~Hz}, 1 \mathrm{H}), 1.80-1.85(\mathrm{~m}, 1 \mathrm{H}), 1.212(\mathrm{~s}, 6 \mathrm{H}), 1.207(\mathrm{~s}, 6 \mathrm{H}), 1.02(\mathrm{~d}, J=$ 
$7.7 \mathrm{~Hz}, 2 \mathrm{H}), 0.89$ (d, $J=6.9 \mathrm{~Hz}, 3 \mathrm{H}) .{ }^{13} \mathrm{C} \mathrm{NMR}\left(101 \mathrm{MHz}, \mathrm{CDCl}_{3}\right) \delta 155.9,139.7,135.8$, $128.5,127.9,127.5,117.1,83.6,80.5,78.0,72.6,40.3,37.3,35.4,25.2,25.1,10.8$. HRMS (ESI ${ }^{+}$): $m / z$ for $\mathrm{C}_{24} \mathrm{H}_{36} \mathrm{~B}_{2} \mathrm{O}_{5} \mathrm{Na}[\mathrm{M}+\mathrm{Na}]^{+}$calcd 449.2647, found 449.2632.

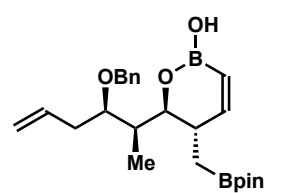

(5S,6S)-6-((2R,3R)-3-(Benzyloxy)hex-5-en-2-yl)-5-((4,4,5,5-tetramet hyl-1,3,2-dioxaborolan-2-yl)methyl)-5,6-dihydro-2H-1,2-oxaborinin -2-ol (15) Prepared according to the general procedure. The crude

mixture was purified by flash column chromatography (hexane : ethyl acetate $=7.5: 1)$ to give the title compound as colorless oil in $82 \%$ yield $(35 \mathrm{mg})$. The $Z / E$ selectivity $(>50: 1)$ and $d r(>30: 1)$ were determined by ${ }^{1} \mathrm{H}$ NMR analysis. $[\alpha]_{\mathrm{D}}{ }^{20}=$ $+29.0^{\circ}\left(\mathrm{c} 0.60, \mathrm{CHCl}_{3}\right) ;{ }^{1} \mathrm{H} \mathrm{NMR}\left(600 \mathrm{MHz}, \mathrm{CDCl}_{3}\right) \delta 7.32-7.36(\mathrm{~m}, 4 \mathrm{H}), 7.26-7.28$ (m, 1H), 6.75 (dd, $J=12.1,2.2 \mathrm{~Hz}, 1 \mathrm{H}), 5.91$ (ddt, $J=17.2,10.1,7.1 \mathrm{~Hz}, 1 \mathrm{H}), 5.60$ (dd, $J=12.1,2.1 \mathrm{~Hz}, 1 \mathrm{H}), 5.10(\mathrm{~d}, J=17.2 \mathrm{~Hz}, 1 \mathrm{H}), 5.06(\mathrm{~d}, J=10.0 \mathrm{~Hz}, 1 \mathrm{H}), 4.59\left(\mathrm{~d}, J_{A B}=\right.$ $11.3 \mathrm{~Hz}, 1 \mathrm{H}), 4.49$ (d, $\left.J_{A B}=11.3 \mathrm{~Hz}, 1 \mathrm{H}\right), 3.96(\mathrm{dd}, J=9.0,2.8 \mathrm{~Hz}, 1 \mathrm{H}), 3.85(\mathrm{~s}, 1 \mathrm{H})$, 3.51 (ddd, $J=6.2,6.2,5.0 \mathrm{~Hz}, 1 \mathrm{H}), 2.59-2.63(\mathrm{~m}, 1 \mathrm{H}), 2.50(\mathrm{ddd}, J=14.3,5.9,5.3 \mathrm{~Hz}$, $1 \mathrm{H}), 2.38(\mathrm{ddd}, J=14.7,6.7,6.7 \mathrm{~Hz}, 1 \mathrm{H}), 1.92-1.96(\mathrm{~m}, 1 \mathrm{H}), 1.24(\mathrm{~s}, 12 \mathrm{H}), 1.01(\mathrm{~d}, J=$ $6.8 \mathrm{~Hz}, 3 \mathrm{H}), 0.91(\mathrm{dd}, J=15.8,5.0 \mathrm{~Hz}, 1 \mathrm{H}), 0.74(\mathrm{dd}, J=15.8,9.7 \mathrm{~Hz}, 1 \mathrm{H}) .{ }^{13} \mathrm{C} \mathrm{NMR}$ $\left(101 \mathrm{MHz}, \mathrm{CDCl}_{3}\right) \delta 158.2,139.3,135.6,128.6,128.1,127.8,117.2,83.7,81.4,79.4$, 72.3, 38.8, 36.4, 35.6, 25.3, 25.1, 10.6. HRMS $\left(\mathrm{ESI}^{+}\right): m / z$ for $\mathrm{C}_{24} \mathrm{H}_{36} \mathrm{~B}_{2} \mathrm{O}_{5} \mathrm{Na}[\mathrm{M}+\mathrm{Na}]^{+}$ calcd 449.2647, found 449.2630.

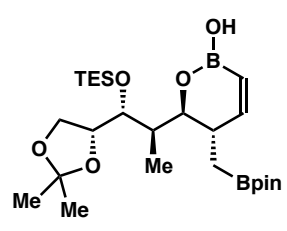

(5S,6S)-6-((1R,2R)-1-((R)-2,2-Dimethyl-1,3-dioxolan-4-yl)-1-((trieth ylsilyl)oxy)propan-2-yl)-5-((4,4,5,5-tetramethyl-1,3,2-dioxaborolan -2-yl)methyl)-5,6-dihydro-2H-1,2-oxaborinin-2-ol (17) Prepared according to the general procedure. The crude mixture was purified by flash column chromatography (hexane : ethyl acetate $=7.5: 1$ ) to give the title compound as colorless oil in 74\% yield (38 mg). The $Z / E$ selectivity $(>50: 1)$ and $d r(>30: 1)$ were determined by ${ }^{1} \mathrm{H}$ NMR analysis. $[\alpha]_{\mathrm{D}}{ }^{20}=+58.9^{\circ}\left(\mathrm{c} 0.55, \mathrm{CHCl}_{3}\right) ;{ }^{1} \mathrm{H}$ NMR $(600 \mathrm{MHz}$, $\left.\mathrm{CDCl}_{3}\right) \delta 6.76(\mathrm{dd}, J=12.1,1.8 \mathrm{~Hz}, 1 \mathrm{H}), 5.60(\mathrm{dd}, J=12.1,2.4 \mathrm{~Hz}, 1 \mathrm{H}), 4.16(\mathrm{ddd}, J=$ 7.3, 6.7, $4.4 \mathrm{~Hz}, 1 \mathrm{H}), 4.06(\mathrm{dd}, J=10.1,1.5 \mathrm{~Hz}, 1 \mathrm{H}), 3.96(\mathrm{dd}, J=7.6,6.6 \mathrm{~Hz}, 1 \mathrm{H}), 3.75$ $-3.79(\mathrm{~m}, 3 \mathrm{H}), 2.55-2.59(\mathrm{~m}, 1 \mathrm{H}), 1.86-1.90(\mathrm{~m}, 1 \mathrm{H}), 1.41(\mathrm{~s}, 3 \mathrm{H}), 1.34(\mathrm{~s}, 3 \mathrm{H}), 1.25$ $(\mathrm{s}, 12 \mathrm{H}), 0.92-0.96(\mathrm{~m}, 10 \mathrm{H}), 0.88(\mathrm{~d}, J=6.9 \mathrm{~Hz}, 3 \mathrm{H}), 0.71(\mathrm{dd}, J=15.8,10.2 \mathrm{~Hz}, 1 \mathrm{H})$, $0.59-0.65(\mathrm{~m}, 6 \mathrm{H}) .{ }^{13} \mathrm{C} \mathrm{NMR}\left(101 \mathrm{MHz}, \mathrm{CDCl}_{3}\right) \delta 158.7,108.7,83.7,78.7,78.2,73.7$, 66.5, 39.3, 36.2, 26.7, 25.8, 25.3, 25.1, 10.5, 7.4, 5.7. HRMS $\left(\mathrm{ESI}^{+}\right): \mathrm{m} / \mathrm{z}$ for $\mathrm{C}_{25} \mathrm{H}_{48} \mathrm{~B}_{2} \mathrm{O}_{7} \mathrm{SiNa}[\mathrm{M}+\mathrm{Na}]^{+}$calcd 533.3253, found 533.3267. 


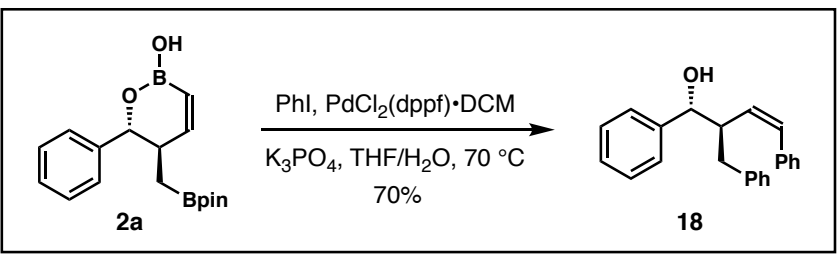

(1R,2R,Z)-2-Benzyl-1,4-diphenylbut-3-en-1-ol (18): In an Ar-filled glove box, vinylboronate $2 \mathrm{a}$ (16 mg, $0.05 \mathrm{mmol}, 1.0$ equiv), iodobenzene (31 mg, $0.15 \mathrm{mmol}, 3.0$ equiv), $\mathrm{PdCl}_{2}$ (dppf).DCM (4 mg, $0.005 \mathrm{mmol}, 10 \mathrm{~mol} \%$ ), $\mathrm{K}_{3} \mathrm{PO}_{4}$ (32 mg, $0.15 \mathrm{mmol}, 3.0$ equiv), THF $(0.5 \mathrm{~mL})$ and a stirring bar were sequentially added into a reaction vial. The vial was sealed with rubber septum and removed from the glove box. Water $(0.1 \mathrm{~mL})$ was added to the mixture under an argon atmosphere. Then the vial was sealed with a cap containing a PTFE-lined silicone septum and kept stirring at $70{ }^{\circ} \mathrm{C}$ with a heating mantle for $12 \mathrm{~h}$. After completion of the reaction, the mixture was filtered through a short pad of Celite. Brine $(1 \mathrm{~mL})$ was added and the mixture was extracted with $\mathrm{Et}_{2} \mathrm{O}(1 \mathrm{~mL} \times 3)$. The combined organic layers were dried over anhydrous $\mathrm{Na}_{2} \mathrm{SO}_{4}$, filtered, and concentrated under reduced pressure. Purification of the crude reaction product was performed by flash column chromatography (hexane : ethyl acetate $=10: 1$ ) to give product $\mathbf{1 8}$ as pale yellow oil in 70\% yield $(11 \mathrm{mg}) .{ }^{1} \mathrm{H}$ NMR $\left(400 \mathrm{MHz}, \mathrm{CDCl}_{3}\right) \delta 7.28-7.36(\mathrm{~m}, 5 \mathrm{H}), 7.12-7.20$ $(\mathrm{m}, 6 \mathrm{H}), 6.98(\mathrm{~d}, J=7.2 \mathrm{~Hz}, 2 \mathrm{H}), 6.72-6.74(\mathrm{~m}, 2 \mathrm{H}), 6.61(\mathrm{~d}, J=11.5 \mathrm{~Hz}, 1 \mathrm{H}), 5.63$ $(\mathrm{dd}, J=11.2,11.2 \mathrm{~Hz}, 1 \mathrm{H}), 4.61(\mathrm{~d}, J=6.0 \mathrm{~Hz}, 1 \mathrm{H}), 3.17-3.25(\mathrm{~m}, 1 \mathrm{H}), 2.75(\mathrm{dd}, J=$ 13.8, $4.8 \mathrm{~Hz}, 1 \mathrm{H}), 2.52(\mathrm{dd}, J=13.6,9.4 \mathrm{~Hz}, 1 \mathrm{H}), 2.06(\mathrm{~s}, 1 \mathrm{H}) .{ }^{13} \mathrm{C}$ NMR $(126 \mathrm{MHz}$, $\left.\mathrm{CDCl}_{3}\right) \delta 142.9,140.0,137.4,133.6,131.9,129.5,128.7,128.54,128.45,128.2,128.1$, 127.2, 126.9, 126.3, 77.1, 47.9, 38.5. HRMS $\left(\mathrm{ESI}^{+}\right): \mathrm{m} / z$ for $\mathrm{C}_{23} \mathrm{H}_{22} \mathrm{ONa}[\mathrm{M}+\mathrm{Na}]^{+}$calcd 337.1568 , found 337.1558 .

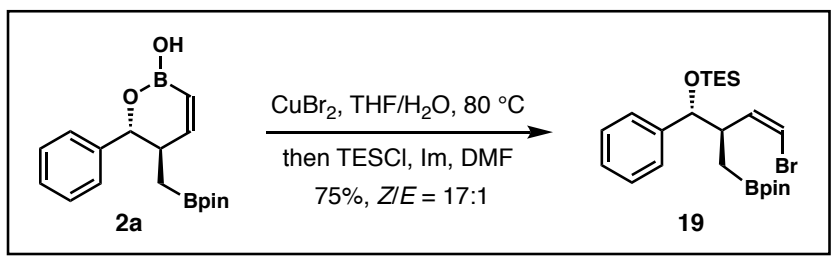

(((1R,2R,Z)-4-Bromo-1-phenyl-2-((4,4,5,5-tetramethyl-1,3,2-dioxaborolan-2-yl)meth yl)but-3-en-1-yl)oxy)triethylsilane (19): To a reaction vial containing a Teflon-coated magnetic stirring bar was added vinylboronate $2 \mathrm{a}(16 \mathrm{mg}, 0.05 \mathrm{mmol}, 1.0$ equiv). THF $(0.3 \mathrm{~mL})$ and $\mathrm{H}_{2} \mathrm{O}(0.3 \mathrm{~mL})$ were added to the vial followed by addition of $\mathrm{CuBr}_{2}(34 \mathrm{mg}$, $0.15 \mathrm{mmol}, 3.0$ equiv). The reaction mixture was stirred at $80{ }^{\circ} \mathrm{C}$ with a heating mantle for $12 \mathrm{~h}$. After cooling to ambient temperature, $\mathrm{H}_{2} \mathrm{O}(0.5 \mathrm{~mL})$ was added to the vial, and 
the mixture was extracted with $\mathrm{Et}_{2} \mathrm{O}(1 \mathrm{~mL}$ x 3$)$. The combined organic layers were dried over anhydrous $\mathrm{Na}_{2} \mathrm{SO}_{4}$, filtered, and concentrated under reduced pressure. The crude reaction product was dissolved in DMF $(0.5 \mathrm{~mL})$. Imidazole $(7 \mathrm{mg}, 0.1 \mathrm{mmol}, 2.0$ equiv) and TESCl (17 $\mu \mathrm{L}, 0.1 \mathrm{mmol}, 2.0$ equiv) were added and the mixture was kept stirring at ambient temperature for $12 \mathrm{~h}$. Water $(5 \mathrm{~mL})$ was added and the mixture was extracted with $\mathrm{Et}_{2} \mathrm{O}(1 \mathrm{~mL} \times 3)$. The combined organic layers were dried over anhydrous $\mathrm{Na}_{2} \mathrm{SO}_{4}$, filtered, and concentrated under reduced pressure. The $Z / E$ ratio was determined by ${ }^{1} \mathrm{H}$ NMR analysis of the crude reaction mixture. Purification of the crude reaction product was performed by flash column chromatography (hexane : ethyl acetate $=30: 1$ ) to give product 19 as colorless oil in $75 \%$ yield $(18 \mathrm{mg}, Z / E=17: 1) .{ }^{1} \mathrm{H}$ NMR $\left(400 \mathrm{MHz}, \mathrm{CDCl}_{3}\right)$ $\delta 7.23-7.26(\mathrm{~m}, 4 \mathrm{H}), 7.19-7.21(\mathrm{~m}, 1 \mathrm{H}), 6.10(\mathrm{~d}, J=7.0 \mathrm{~Hz}, 1 \mathrm{H}), 5.98(\mathrm{dd}, J=9.4,7.5$ $\mathrm{Hz}, 1 \mathrm{H}), 4.73$ (d, $J=4.6 \mathrm{~Hz}, 1 \mathrm{H}), 3.07-3.14(\mathrm{~m}, 1 \mathrm{H}), 1.23(\mathrm{~s}, 12 \mathrm{H}), 1.06$ (dd, $J=15.3$, $5.3 \mathrm{~Hz}, 1 \mathrm{H}), 0.87$ (t, $J=7.9 \mathrm{~Hz}, 9 \mathrm{H}), 0.77(\mathrm{dd}, J=15.3,9.3 \mathrm{~Hz}, 1 \mathrm{H}), 0.47-0.54(\mathrm{~m}, 6 \mathrm{H})$. ${ }^{13} \mathrm{C}$ NMR (101 MHz, $\left.\mathrm{CDCl}_{3}\right) \delta 143.7,137.0,127.9,127.2,127.1,108.2,83.5,77.9,44.9$, 25.23, 25.19, 7.2, 5.2. HRMS (ESI $\left.{ }^{+}\right): m / z$ for $\mathrm{C}_{23} \mathrm{H}_{38} \mathrm{BBrO}_{3} \mathrm{SiNa}[\mathrm{M}+\mathrm{Na}]^{+}$calcd 503.1764, found 503.1747 .

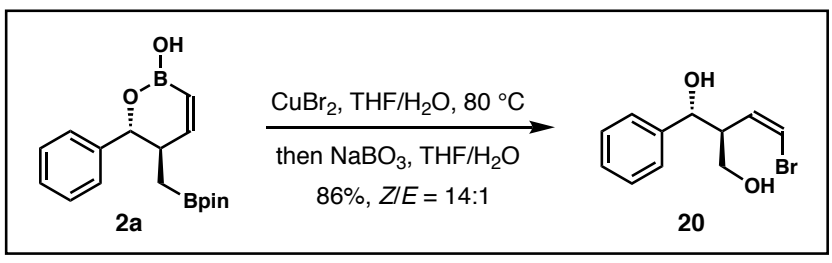

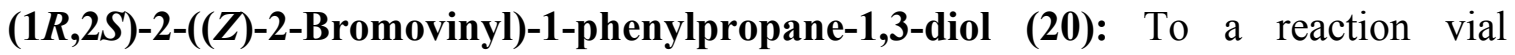
containing a Teflon-coated magnetic stirring bar was added vinylboronate 2a (16 mg, 0.1 mmol, 1.0 equiv). THF $(0.5 \mathrm{~mL})$ and $\mathrm{H}_{2} \mathrm{O}(0.5 \mathrm{~mL})$ were added to the vial followed by addition of $\mathrm{CuBr}_{2}(67 \mathrm{mg}, 0.3 \mathrm{mmol}, 3.0$ equiv). The reaction mixture was stirred at $80{ }^{\circ} \mathrm{C}$ with a heating mantle for $12 \mathrm{~h}$. After cooling to ambient temperature, $\mathrm{H}_{2} \mathrm{O}(0.5 \mathrm{~mL})$ was added to the vial, and the mixture was extracted with $\mathrm{Et}_{2} \mathrm{O}(1 \mathrm{~mL} \times 3)$. The combined organic layers were concentrated under reduced pressure. Then the crude reaction mixture was dissolved in THF $(0.5 \mathrm{~mL})$. A solution of $\mathrm{NaBO}_{3} \cdot 4 \mathrm{H}_{2} \mathrm{O}(31 \mathrm{mg}, 0.2 \mathrm{mmol}$, 2.0 equiv) in water $(0.5 \mathrm{~mL})$ was added. The reaction mixture was kept stirring at ambient temperature for $18 \mathrm{~h}$. The organic layer was separated and the aqueous layer was extracted with EtOAc (1 mL x 3). The combined organic layers were dried over anhydrous $\mathrm{Na}_{2} \mathrm{SO}_{4}$, filtered, and concentrated under reduced pressure. The $Z / E$ ratio was determined by ${ }^{1} \mathrm{H}$ NMR analysis of the crude reaction mixture. Purification of the crude reaction product was performed by flash column chromatography (hexane : ethyl acetate 
$=2: 1)$ to give product 20 as colorless oil in $86 \%$ yield $(22 \mathrm{mg}, Z / E=14: 1) .{ }^{1} \mathrm{H}$ NMR (400 $\left.\mathrm{MHz}, \mathrm{CDCl}_{3}\right) \delta 7.34-7.36(\mathrm{~m}, 4 \mathrm{H}), 7.27-7.31(\mathrm{~m}, 1 \mathrm{H}), 6.33(\mathrm{~d}, J=7.2 \mathrm{~Hz}, 1 \mathrm{H}), 6.23$ (dd, $J=9.1,7.3 \mathrm{~Hz}, 1 \mathrm{H}), 5.08(\mathrm{~d}, J=3.6 \mathrm{~Hz}, 1 \mathrm{H}), 3.75-3.85(\mathrm{~m}, 2 \mathrm{H}), 3.16-3.22(\mathrm{~m}$, $1 \mathrm{H}), 2.68(\mathrm{~s}, 1 \mathrm{H}), 2.04(\mathrm{~s}, 1 \mathrm{H}) .{ }^{13} \mathrm{C} \mathrm{NMR}\left(101 \mathrm{MHz}, \mathrm{CDCl}_{3}\right) \delta 142.2,131.4,128.7,128.1$, 126.4, 111.7, 75.2, 64.2, 49.4. HRMS $\left(\mathrm{ESI}^{+}\right): m / z$ for $\mathrm{C}_{11} \mathrm{H}_{13} \mathrm{BrO}_{2} \mathrm{Na}[\mathrm{M}+\mathrm{Na}]^{+}$calcd 278.9997 , found 278.9984 .

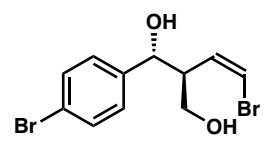

(1R,2S)-1-(4-Bromophenyl)-2-((Z)-2-bromovinyl)propane-1,3-diol (SI-I): Prepared from vinylboronate $\mathbf{2 f}$ according to the above procedure. The crude mixture was purified by column chromatography (hexane : ethyl acetate $=5: 1)$ to give the title compound as colorless oil in $83 \%$ yield $(28 \mathrm{mg}, Z / E=$ 25:1). ${ }^{1} \mathrm{H}$ NMR (600 MHz, $\left.\mathrm{CDCl}_{3}\right) \delta 7.46(\mathrm{~d}, J=8.4 \mathrm{~Hz}, 2 \mathrm{H}), 7.23(\mathrm{~d}, J=8.4 \mathrm{~Hz}, 2 \mathrm{H})$, $6.33(\mathrm{~d}, J=7.2 \mathrm{~Hz}, 1 \mathrm{H}), 6.22(\mathrm{dd}, J=9.3,7.2 \mathrm{~Hz}, 1 \mathrm{H}), 5.09(\mathrm{~d}, J=3.9 \mathrm{~Hz}, 1 \mathrm{H}), 3.85(\mathrm{dd}$, $J=10.6,5.8 \mathrm{~Hz}, 1 \mathrm{H}), 3.81$ (dd, $J=10.6,4.4 \mathrm{~Hz}, 1 \mathrm{H}), 3.12-3.16$ (m, 1H), 2.81 (brs, $1 \mathrm{H}$ ), 1.94 (brs, 1H). ${ }^{13} \mathrm{C}$ NMR $\left(151 \mathrm{MHz}, \mathrm{CDCl}_{3}\right) \delta 141.4,131.7,130.8,128.1,121.8,112.0$, 74.5, 64.3, 49.2. HRMS $\left(\mathrm{ESI}^{+}\right): \mathrm{m} / z$ for $\mathrm{C}_{11} \mathrm{H}_{12} \mathrm{Br}_{2} \mathrm{O}_{2} \mathrm{Na}[\mathrm{M}+\mathrm{Na}]^{+}$calcd 356.9102, found 356.9112 .

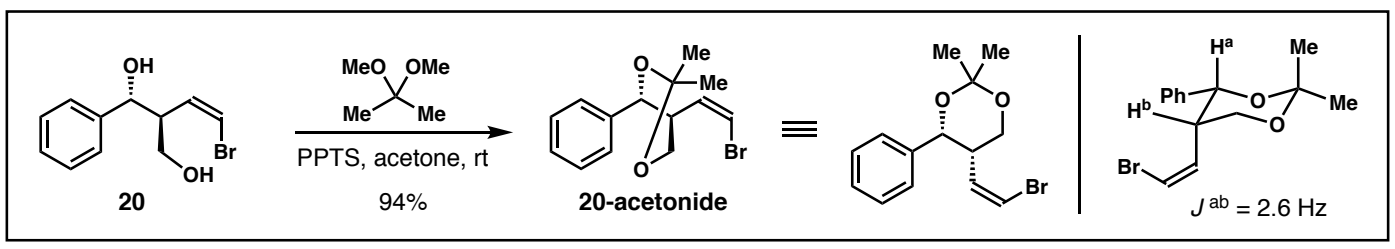

(4R,5S)-5-((Z)-2-Bromovinyl)-2,2-dimethyl-4-phenyl-1,3-dioxane (20-acetonide): To a solution of diol $20(0.05 \mathrm{mmol})$ in acetone $(0.2 \mathrm{~mL})$ were added 2,2-dimethoxypropane $(0.2 \mathrm{~mL})$ and PPTS $(1 \mathrm{mg})$. After stirring at ambient temperature for $12 \mathrm{~h}$, the reaction mixture was filtered through a pad of silica gel and the filtrate was concentrated under reduced pressure. Purification of the crude product was performed by flash column chromatography (hexane : ethyl acetate $=20: 1$ ) to give product 20-acetonide in $94 \%$ yield $(14 \mathrm{mg})$ as a white solid. The $J$ value $(2.6 \mathrm{~Hz})$ is consistent with a syn-relative stereochemistry. ${ }^{1} \mathrm{H}$ NMR $\left(600 \mathrm{MHz}, \mathrm{CDCl}_{3}\right) \delta 7.29-7.33(\mathrm{~m}, 4 \mathrm{H}), 7.22-7.25(\mathrm{~m}, 1 \mathrm{H})$, 6.47 (dd, $J=9.3,7.1 \mathrm{~Hz}, 1 \mathrm{H}), 6.10(\mathrm{~d}, J=7.1 \mathrm{~Hz}, 1 \mathrm{H}), 5.24(\mathrm{~d}, J=2.6 \mathrm{~Hz}, 1 \mathrm{H}), 4.35$ (dd, $J=11.5,2.7 \mathrm{~Hz}, 1 \mathrm{H}), 3.87(\mathrm{dd}, J=11.5,1.6 \mathrm{~Hz}, 1 \mathrm{H}), 2.96-2.98(\mathrm{~m}, 1 \mathrm{H}), 1.60(\mathrm{~s}, 3 \mathrm{H})$, $1.55(\mathrm{~s}, 3 \mathrm{H}) .{ }^{13} \mathrm{C} \mathrm{NMR}\left(151 \mathrm{MHz}, \mathrm{CDCl}_{3}\right) \delta 140.3,132.4,128.4,127.5,125.8,109.5$, 99.8, 72.4, 64.9, 41.4, 30.1, 19.3. HRMS $\left(\mathrm{EI}^{+}\right): \mathrm{m} / \mathrm{z}$ for $\mathrm{C}_{13} \mathrm{H}_{14} \mathrm{O}_{2} \mathrm{Br}\left[\mathrm{M}-\mathrm{CH}_{3}\right]^{+}$calcd 281.0177 , found 281.0180 . 


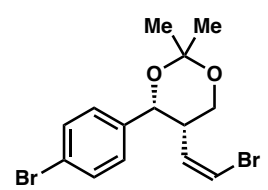

(4R,5S)-4-(4-Bromophenyl)-5-((Z)-2-bromovinyl)-2,2-dimethyl-1,3-d ioxane (SI-I-acetonide): Prepared from SI-I according to the procedure for the synthesis of $\mathbf{2 0 - a c e t o n i d e . ~ T h e ~ c r u d e ~ m i x t u r e ~ w a s ~ p u r i f i e d ~ b y ~}$ column chromatography (hexane : ethyl acetate $=20: 1$ ) to give

SI-I-acetonide as colorless oil in $85 \%$ yield $(16 \mathrm{mg}) .{ }^{1} \mathrm{H}$ NMR $\left(600 \mathrm{MHz}, \mathrm{CDCl}_{3}\right) \delta 7.42$ - $7.44(\mathrm{~m}, 2 \mathrm{H}), 7.17(\mathrm{~d}, J=8.4 \mathrm{~Hz}, 2 \mathrm{H}), 6.42(\mathrm{dd}, J=9.4,7.2 \mathrm{~Hz}, 1 \mathrm{H}), 6.11$ (d, $J=7.1$ $\mathrm{Hz}, 1 \mathrm{H}), 5.18(\mathrm{~d}, J=2.6 \mathrm{~Hz}, 1 \mathrm{H}), 4.33(\mathrm{dd}, J=11.6,2.7 \mathrm{~Hz}, 1 \mathrm{H}), 3.85$ (dd, $J=11.6,1.6$ $\mathrm{Hz}, 1 \mathrm{H}), 2.93-2.95(\mathrm{~m}, 1 \mathrm{H}), 1.58(\mathrm{~s}, 3 \mathrm{H}), 1.53(\mathrm{~s}, 3 \mathrm{H}) .{ }^{13} \mathrm{C} \mathrm{NMR}\left(151 \mathrm{MHz}, \mathrm{CDCl}_{3}\right) \delta$ 139.4, 131.9, 131.5, 127.6, 121.3, 109.9, 99.9, 71.9, 64.7, 41.2, 30.0, 19.3. HRMS (EI $\left.{ }^{+}\right)$: $\mathrm{m} / \mathrm{z}$ for $\mathrm{C}_{13} \mathrm{H}_{13} \mathrm{O}_{2} \mathrm{Br}_{2}\left[\mathrm{M}-\mathrm{CH}_{3}\right]^{+}$calcd 358.9282 , found 358.9274 . The syn-relative stereochemistry was confirmed by the $J$ value $(2.6 \mathrm{~Hz})$.

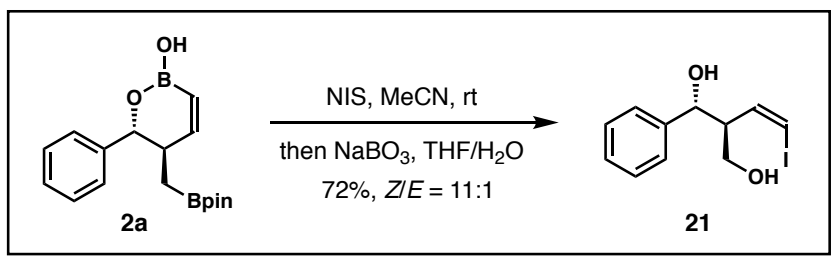

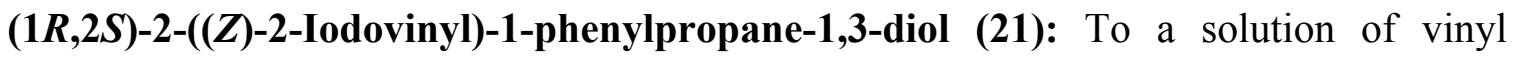
boronate $2 \mathrm{a}$ (16 $\mathrm{mg}, 0.05 \mathrm{mmol}, 1.0$ equiv) in acetonitrile $(0.5 \mathrm{~mL})$ was added $N$-iodosuccinimide (NIS, $23 \mathrm{mg}, 0.1 \mathrm{mmol}, 2.0$ equiv). The reaction mixture was protected from light and stirred for $2 \mathrm{~h}$ at ambient temperature. Then a saturated aqueous $\mathrm{Na}_{2} \mathrm{~S}_{2} \mathrm{O}_{3}$ solution $(2 \mathrm{~mL}$ ) was added and the mixture was kept stirring for $15 \mathrm{~min}$. The mixture was extracted with $\mathrm{Et}_{2} \mathrm{O}(2 \mathrm{~mL}$ x 3$)$. The combined organic extracts were washed with saturated $\mathrm{NaHCO}_{3}$ and brine, dried over anhydrous $\mathrm{Na}_{2} \mathrm{SO}_{4}$, filtered, and concentrated under reduced pressure. The crude reaction product was dissolved in THF $(0.5 \mathrm{~mL})$, a solution of $\mathrm{NaBO}_{3} \cdot 4 \mathrm{H}_{2} \mathrm{O}(16 \mathrm{mg}, 0.1 \mathrm{mmol}, 2.0$ equiv) in water $(0.5 \mathrm{~mL})$ was added. The reaction mixture was stirred at ambient temperature for $15 \mathrm{~h}$. The organic layer was separated and the aqueous layer was extracted with EtOAc ( $1 \mathrm{~mL} \times 3)$. The combined organic layers were dried over anhydrous $\mathrm{Na}_{2} \mathrm{SO}_{4}$, filtered, and concentrated under reduced pressure. Purification of the crude product was performed by flash column chromatography (hexane : ethyl acetate $=2: 1)$ to give product $\mathbf{2 1}$ in $72 \%$ yield $(11 \mathrm{mg}$, $Z / E=11: 1) .{ }^{1} \mathrm{H}$ NMR $\left(400 \mathrm{MHz}, \mathrm{CDCl}_{3}\right) \delta 7.34-7.36(\mathrm{~m}, 4 \mathrm{H}), 7.27-7.31(\mathrm{~m}, 1 \mathrm{H})$, $6.44(\mathrm{~d}, J=7.6 \mathrm{~Hz}, 1 \mathrm{H}), 6.31$ (dd, $J=9.1,7.7 \mathrm{~Hz}, 1 \mathrm{H}), 5.10$ (app. s, 1H), $3.75-3.86$ (m, 2H), $3.00-3.05(\mathrm{~m}, 1 \mathrm{H}), 2.63(\mathrm{~s}, 1 \mathrm{H}), 1.99(\mathrm{~s}, 1 \mathrm{H}) .{ }^{13} \mathrm{C} \mathrm{NMR}\left(101 \mathrm{MHz}, \mathrm{CDCl}_{3}\right) \delta 142.2$, $137.9,128.7,128.1,126.5,86.7,75.2,64.1,53.8$. HRMS $\left(\mathrm{ESI}^{+}\right): \mathrm{m} / z$ for $\mathrm{C}_{11} \mathrm{H}_{13} \mathrm{IO}_{2} \mathrm{Na}$ $[\mathrm{M}+\mathrm{Na}]^{+}$calcd 326.9858 , found 326.9845 . 


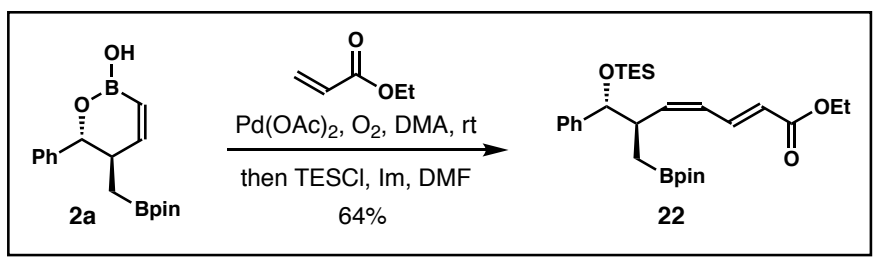

\section{Ethyl (2E,4Z,6R,7R)-7-phenyl-6-((4,4,5,5-tetramethyl-1,3,2-dioxaborolan-2-yl)methy} l)-7-((triethylsilyl)oxy)hepta-2,4-dienoate (22): To a solution of ethyl acrylate (16 $\mu \mathrm{L}$, $0.15 \mathrm{mmol}, 3.0$ equiv) in $N, N$-dimethylacetamide (DMA, $0.25 \mathrm{~mL}$ ) were added vinylboronate $2 \mathrm{a}$ (16 mg, $0.05 \mathrm{mmol}, 1.0$ equiv) and $\mathrm{Pd}(\mathrm{OAc})_{2}(1.1 \mathrm{mg}, 0.005 \mathrm{mmol}, 10$ mol \%). The reaction vial was fitted with an oxygen balloon. The mixture was stirred at ambient temperature for $24 \mathrm{~h}$. After completion of the reaction, $\mathrm{Et}_{2} \mathrm{O}(3 \mathrm{~mL})$ was added, and the reaction mixture was washed with water $(5 \mathrm{~mL} \times 3)$. The organic layer was dried over anhydrous $\mathrm{Na}_{2} \mathrm{SO}_{4}$, filtered and concentrated under reduced pressure. The crude reaction product was dissolved in DMF $(0.5 \mathrm{~mL})$. Imidazole $(7 \mathrm{mg}, 0.1 \mathrm{mmol}, 2.0$ equiv) and TESCl ( $17 \mu \mathrm{L}, 0.1 \mathrm{mmol}, 2.0$ equiv) were added. The reaction mixture was kept stirring at ambient temperature for $12 \mathrm{~h}$. Then water $(5 \mathrm{~mL})$ was added and the mixture was extracted with $\mathrm{Et}_{2} \mathrm{O}(1 \mathrm{~mL} \times 3)$. The combined organic layers were dried over anhydrous $\mathrm{Na}_{2} \mathrm{SO}_{4}$, filtered, and concentrated under reduced pressure. Purification of the crude reaction product was performed by flash column chromatography (hexane : ethyl acetate $=10: 1)$ to give product 22 as colorless oil in $64 \%$ yield $(16 \mathrm{mg}) .{ }^{1} \mathrm{H}$ NMR (400 $\left.\mathrm{MHz} \mathrm{CDCl}_{3}\right) \delta 7.40(\mathrm{dd}, J=14.8,11.8 \mathrm{~Hz}, 1 \mathrm{H}), 7.26-7.28(\mathrm{~m}, 4 \mathrm{H}), 7.18-7.21(\mathrm{~m}$, $1 \mathrm{H}), 6.07(\mathrm{dd}, J=11.2,11.2 \mathrm{~Hz}, 1 \mathrm{H}), 5.71-5.76(\mathrm{~m}, 2 \mathrm{H}), 4.63(\mathrm{~d}, J=5.3 \mathrm{~Hz}, 1 \mathrm{H}), 4.20$ $(\mathrm{q}, J=7.1 \mathrm{~Hz}, 2 \mathrm{H}), 3.06-3.14(\mathrm{~m}, 1 \mathrm{H}), 1.30(\mathrm{t}, J=7.1 \mathrm{~Hz}, 3 \mathrm{H}), 1.20(\mathrm{~s}, 6 \mathrm{H}), 1.18$ (s, $6 \mathrm{H}), 0.98(\mathrm{dd}, J=15.3,4.7 \mathrm{~Hz}, 1 \mathrm{H}), 0.85$ (t, $J=7.9 \mathrm{~Hz}, 9 \mathrm{H}), 0.79$ (dd, $J=15.6,9.8 \mathrm{~Hz}$, $1 \mathrm{H}), 0.48(\mathrm{q}, J=8.1 \mathrm{~Hz}, 6 \mathrm{H}) .{ }^{13} \mathrm{C} \mathrm{NMR}\left(101 \mathrm{MHz}, \mathrm{CDCl}_{3}\right) \delta 167.5,143.8,143.2,141.1$, $128.0,127.4,127.3,127.1,121.3,83.5,79.2,60.4,43.4,25.2,14.7,7.1,5.2$. HRMS $\left(\mathrm{ESI}^{+}\right): m / z$ for $\mathrm{C}_{28} \mathrm{H}_{45} \mathrm{BO}_{5} \mathrm{SiNa}[\mathrm{M}+\mathrm{Na}]^{+}$calcd 523.3027, found 523.3044.

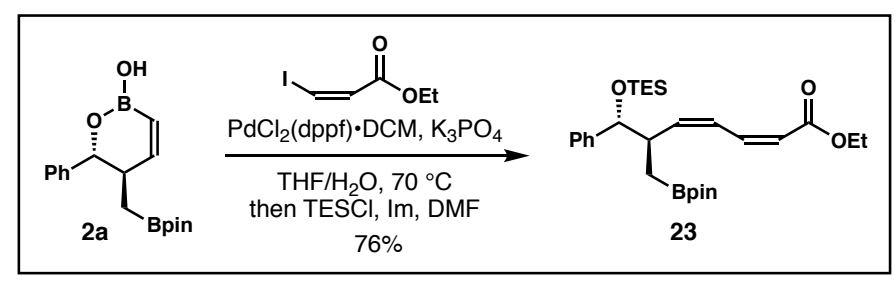

Ethyl (2Z,4Z,6R,7R)-7-phenyl-6-((4,4,5,5-tetramethyl-1,3,2-dioxaborolan-2-yl)methy 1)-7-((triethylsilyl)oxy)hepta-2,4-dienoate (23): In an Ar-filled glove box, vinylboronate 2a (16 mg, $0.05 \mathrm{mmol}, 1.0$ equiv), vinyl iodide (15 mg, $0.065 \mathrm{mmol}, 1.3$ equiv), 
$\mathrm{PdCl}_{2}$ (dppf).DCM (4 mg, $0.005 \mathrm{mmol}, 10 \mathrm{~mol} \%$ ), $\mathrm{K}_{3} \mathrm{PO}_{4}(14 \mathrm{mg}, 0.065 \mathrm{mmol}, 1.3$ equiv), THF $(0.5 \mathrm{~mL})$ and a stirring bar were sequentially added into a reaction vial. The vial was sealed with rubber septum and removed from the glove box. Then water $(0.1 \mathrm{~mL})$ was added to the mixture under an argon atmosphere. The vial was sealed with a cap containing a PTFE-lined silicone septum and stirred at $70{ }^{\circ} \mathrm{C}$ with a heating mantle for 12 h. After completion of the reaction, the mixture was filtered through a short pad of Celite. Brine $(1 \mathrm{~mL})$ was added and the mixture was extracted with $\mathrm{Et}_{2} \mathrm{O}(1 \mathrm{~mL} \times 3)$. The combined organic layers were dried over anhydrous $\mathrm{Na}_{2} \mathrm{SO}_{4}$, filtered, and concentrated under reduced pressure. The crude reaction product was dissolved in DMF $(0.5 \mathrm{~mL})$. Imidazole ( $7 \mathrm{mg}, 0.1 \mathrm{mmol}, 2.0$ equiv) and TESCl (17 $\mu \mathrm{L}, 0.1 \mathrm{mmol}, 2.0$ equiv) were added, and the mixture was kept stirring at ambient temperature for $12 \mathrm{~h}$. Water $(5 \mathrm{~mL})$ was added and the mixture was extracted with $\mathrm{Et}_{2} \mathrm{O}(1 \mathrm{~mL}$ x 3$)$. The combined organic layers were dried over anhydrous $\mathrm{Na}_{2} \mathrm{SO}_{4}$, filtered, and concentrated under reduced pressure. Purification of the crude reaction product was performed by flash column chromatography (hexane : ethyl acetate $=10: 1$ ) to give product 23 as colorless oil in $76 \%$ yield (19 mg). ${ }^{1} \mathrm{H}$ NMR (400 MHz, $\left.\mathrm{CDCl}_{3}\right) \delta 7.26-7.28(\mathrm{~m}, 5 \mathrm{H}), 7.20-7.23(\mathrm{~m}, 1 \mathrm{H})$, $6.77(\mathrm{dd}, J=11.7,11.7 \mathrm{~Hz}, 1 \mathrm{H}), 5.77(\mathrm{dd}, J=10.8,10.8 \mathrm{~Hz}, 1 \mathrm{H}), 5.54$ (d, $J=11.5 \mathrm{~Hz}$, $1 \mathrm{H}), 4.60(\mathrm{~d}, J=5.3 \mathrm{~Hz}, 1 \mathrm{H}), 4.18(\mathrm{q}, J=7.1 \mathrm{~Hz}, 2 \mathrm{H}), 3.04-3.11(\mathrm{~m}, 1 \mathrm{H}), 1.30$ (t, $J=$ $7.1 \mathrm{~Hz}, 3 \mathrm{H}), 1.20$ (s, 6H), $1.18(\mathrm{~s}, 6 \mathrm{H}), 0.96$ (dd, $J=15.3,4.4 \mathrm{~Hz}, 1 \mathrm{H}), 0.85$ (t, $J=7.9 \mathrm{~Hz}$, $9 \mathrm{H}), 0.78(\mathrm{dd}, J=15.5,9.9 \mathrm{~Hz}, 1 \mathrm{H}), 0.48(\mathrm{q}, J=7.8 \mathrm{~Hz}, 6 \mathrm{H}) .{ }^{13} \mathrm{C}$ NMR $(101 \mathrm{MHz}$, $\left.\mathrm{CDCl}_{3}\right) \delta 166.9,143.9,143.3,140.6,128.0,127.3,127.1,125.3,117.2,83.5,79.3,60.1$, 42.6, 25.2, 14.6, 7.1, 5.2. HRMS $\left(\mathrm{ESI}^{+}\right): \mathrm{m} / \mathrm{z}$ for $\mathrm{C}_{28} \mathrm{H}_{45} \mathrm{BO}_{5} \mathrm{SiNa}[\mathrm{M}+\mathrm{Na}]^{+}$calcd 523.3027, found 523.3038.

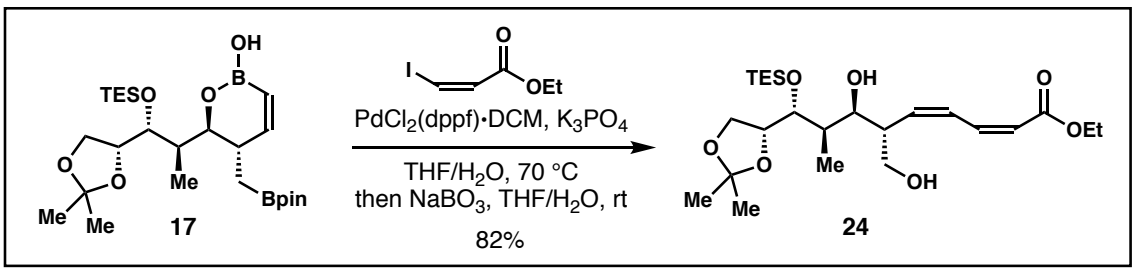

Ethyl $(2 Z, 4 Z, 6 R, 7 S, 8 R, 9 R)-9-((R)-2,2-d i m e t h y l-1,3-d i o x o l a n-4-y l)-7-h y d r o x y-6-(h y d r$ oxymethyl)-8-methyl-9-((triethylsilyl)oxy)nona-2,4-dienoate (24): In an Ar-filled glove box, vinylboronate 17 (41 $\mathrm{mg}, 0.08 \mathrm{mmol}, 1.0$ equiv), vinyl iodide (23 $\mathrm{mg}, 0.1$ mmol, 1.3 equiv), $\mathrm{PdCl}_{2}$ (dppf).DCM (7 mg, $0.008 \mathrm{mmol}, 10 \mathrm{~mol} \%$ ), $\mathrm{K}_{3} \mathrm{PO}_{4}(22 \mathrm{mg}, 0.1$ mmol, 1.3 equiv), THF $(0.5 \mathrm{~mL})$ and a stirring bar were sequentially added into a reaction vial. The vial was sealed with rubber septum and removed from the glove box. Then water $(0.1 \mathrm{~mL})$ was added to the mixture under an argon atmosphere. The vial was 
sealed with a cap containing a PTFE-lined silicone septum and kept stirring at $70{ }^{\circ} \mathrm{C}$ for $12 \mathrm{~h}$. After completion of the reaction, the mixture was filtered through a short pad of Celite. Brine $(1 \mathrm{~mL})$ was added and the mixture was extracted with $\mathrm{Et}_{2} \mathrm{O}(1 \mathrm{~mL} \times 3)$. The combined organic layers were concentrated under reduced pressure. The crude reaction product was dissolved in THF $(0.5 \mathrm{~mL})$, and a solution of $\mathrm{NaBO}_{3} \cdot 4 \mathrm{H}_{2} \mathrm{O}(25 \mathrm{mg}, 0.16$ mmol, 2.0 equiv) in water $(0.5 \mathrm{~mL})$ was added. The reaction mixture was stirred at ambient temperature for $16 \mathrm{~h}$. The organic layer was separated and the aqueous layer was extracted with EtOAc (1 mL x 3). The combined organic layers were dried over anhydrous $\mathrm{Na}_{2} \mathrm{SO}_{4}$, filtered, and concentrated under reduced pressure. Purification of the crude reaction product was performed by flash column chromatography (hexane : ethyl acetate $=2: 1)$ to give product 24 as colorless oil in $82 \%$ yield $(31 \mathrm{mg}) .{ }^{1} \mathrm{H}$ NMR (600 $\left.\mathrm{MHz}, \mathrm{CDCl}_{3}\right) \delta 7.53(\mathrm{dd}, J=11.6,11.6 \mathrm{~Hz}, 1 \mathrm{H}), 6.91$ (ddd, $\left.J=11.8,11.8,0.8 \mathrm{~Hz}, 1 \mathrm{H}\right)$, $5.99-6.03(\mathrm{~m}, 1 \mathrm{H}), 5.72(\mathrm{~d}, J=11.5 \mathrm{~Hz}, 1 \mathrm{H}), 4.25(\mathrm{ddd}, J=8.5,8.5,6.1 \mathrm{~Hz}, 1 \mathrm{H}), 4.18$ (q, $J=7.1 \mathrm{~Hz}, 2 \mathrm{H}), 4.10$ (app. d, $J=6.9 \mathrm{~Hz}, 1 \mathrm{H}), 3.97$ (dd, $J=8.0,6.1 \mathrm{~Hz}, 1 \mathrm{H}), 3.79$ (s, $1 \mathrm{H}), 3.69$ (dd, $J=8.1,1.8 \mathrm{~Hz}, 1 \mathrm{H}), 3.64(\mathrm{ddd}, J=10.1,6.4,5.8 \mathrm{~Hz}, 1 \mathrm{H}), 3.54$ (ddd, $J=$ $10.0,6.0,3.3 \mathrm{~Hz}, 1 \mathrm{H}), 3.44(\mathrm{dd}, J=8.5,8.4 \mathrm{~Hz}, 1 \mathrm{H}), 2.87-2.91(\mathrm{~m}, 1 \mathrm{H}), 1.71$ (dd, $J=$ 6.2, 4.7 Hz, 1H), $1.49-1.52(\mathrm{~m}, 1 \mathrm{H}), 1.40(\mathrm{~s}, 3 \mathrm{H}), 1.35(\mathrm{~s}, 3 \mathrm{H}), 1.29(\mathrm{t}, J=7.1 \mathrm{~Hz}, 3 \mathrm{H})$, $1.11(\mathrm{~d}, J=7.0 \mathrm{~Hz}, 3 \mathrm{H}), 0.96(\mathrm{t}, J=8.0 \mathrm{~Hz}, 9 \mathrm{H}), 0.60-0.73(\mathrm{~m}, 6 \mathrm{H}) .{ }^{13} \mathrm{C} \mathrm{NMR}(151$ $\left.\mathrm{MHz}, \mathrm{CDCl}_{3}\right) \delta 166.7,139.4,138.9,127.6,119.3,109.7,81.6,78.9,72.1,66.4,64.3$, 60.4, 44.3, 37.6, 27.0, 25.9, 14.6, 12.4, 7.2, 5.4. HRMS $\left(\mathrm{ESI}^{+}\right): \mathrm{m} / z$ for $\mathrm{C}_{24} \mathrm{H}_{45} \mathrm{O}_{7} \mathrm{Si}$ $[\mathrm{M}+\mathrm{H}]^{+}$calcd 473.2935, found 473.2930.

\section{Synthesis of diphenylation products SI-II for the assignment of enantiomeric excess of product 2:}

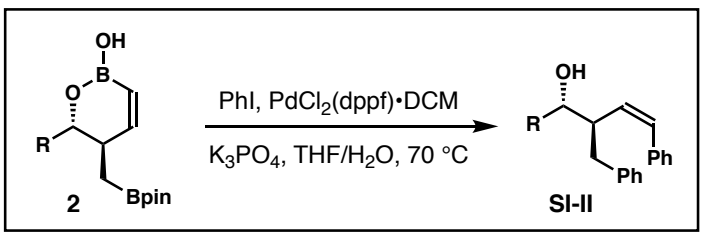

Diphenylation products SI-II were synthesized via Suzuki coupling from compounds 2 using the procedure for the synthesis of compound 18. Products SI-II were used for the assignment of enantiomeric excess of compounds 2 by HPLC analyses. 
Assignment of the absolute configuration of the secondary alcohol group of diphenylation products derived from $2 \mathrm{~d}$ and $2 \mathrm{k}$ using Mosher ester analysis: ${ }^{2}$

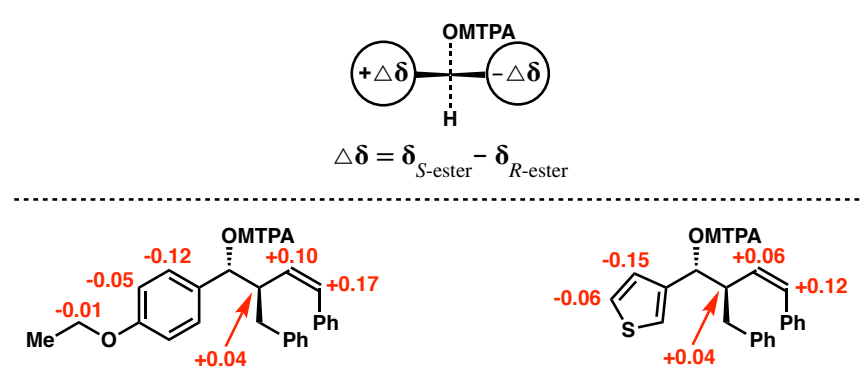

\section{References:}

1. Diene 3 was prepared according to the literature procedure: (a) Hata, T.; Kitagawa, H.; Masai, H.; Kurahashi, T.; Shimizu, M.; Hiyama, T. Angew. Chem. Int. Ed. 2001, 40, 790. (b) Kurahashi, T.; Hata, T.; Masai, H.; Kitagawa, H.; Shimizu, M.; Hiyama, T. Tetrahedron 2002, 58, 6381.

2. (a) Dale, J. A.; Mosher, H. S. J. Am. Chem. Soc. 1973, 95, 512. (b) Ohtani, I.; Kusumi, T.; Kashman, Y.; Kakisawa, H. J. Am. Chem. Soc. 1991, 113, 4092. (c) Hoye, T. R.; Jeffrey, C. S.; Shao, F. Nat. Protoc. 2007, 2, 2451. 

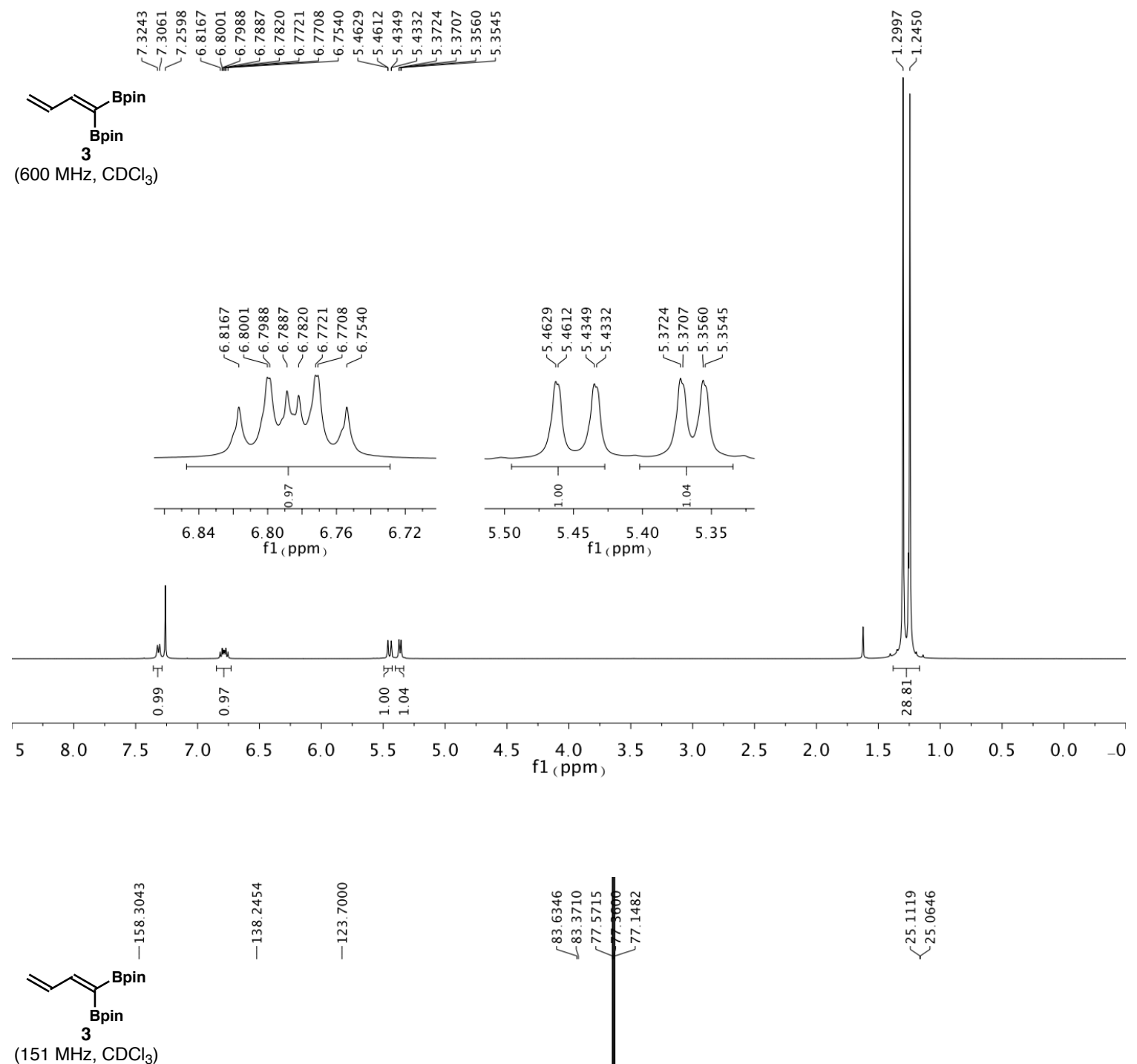

콩ํㅀ

崩

(151 MHz, $\mathrm{CDCl}_{3}$ )

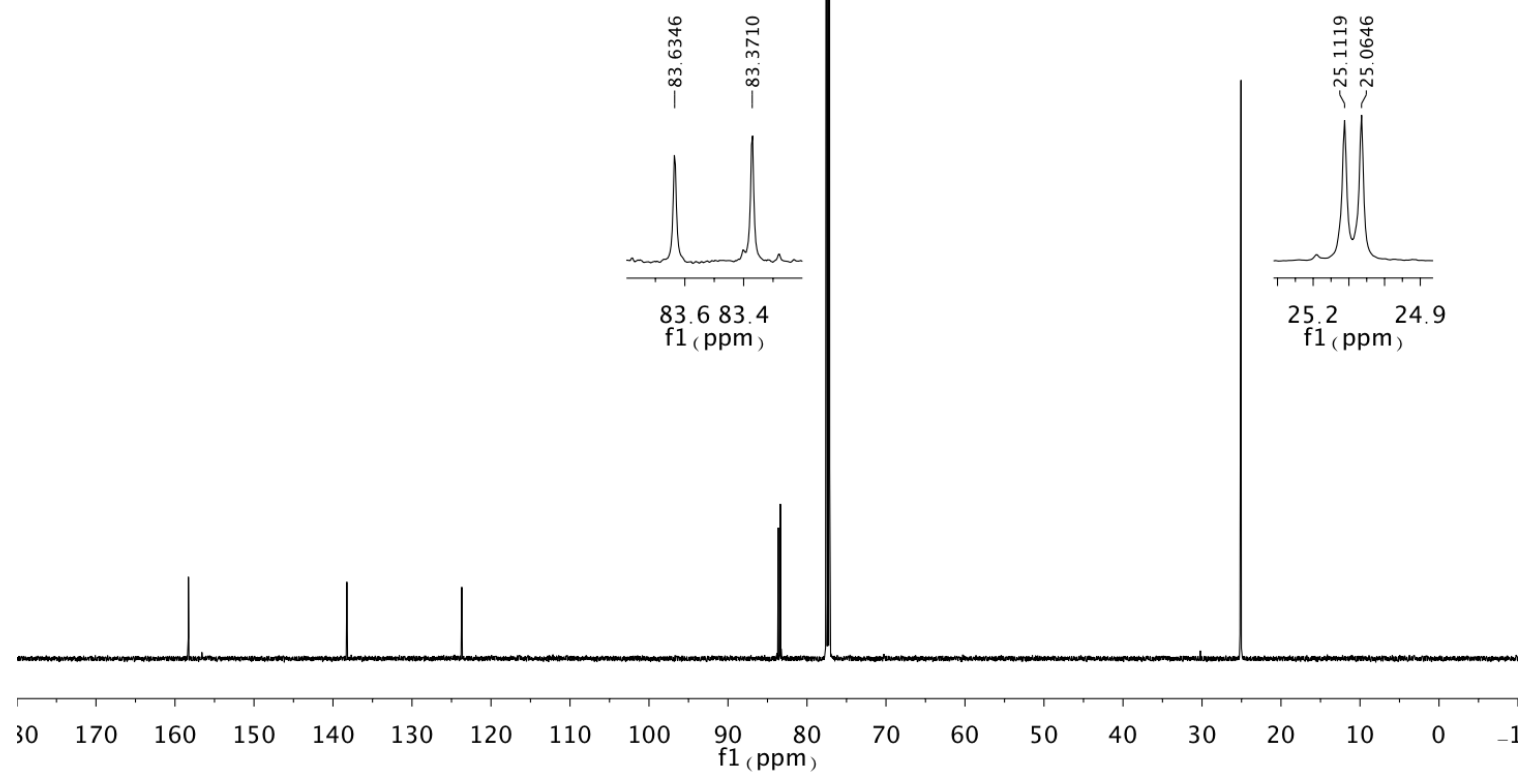

SI-23 

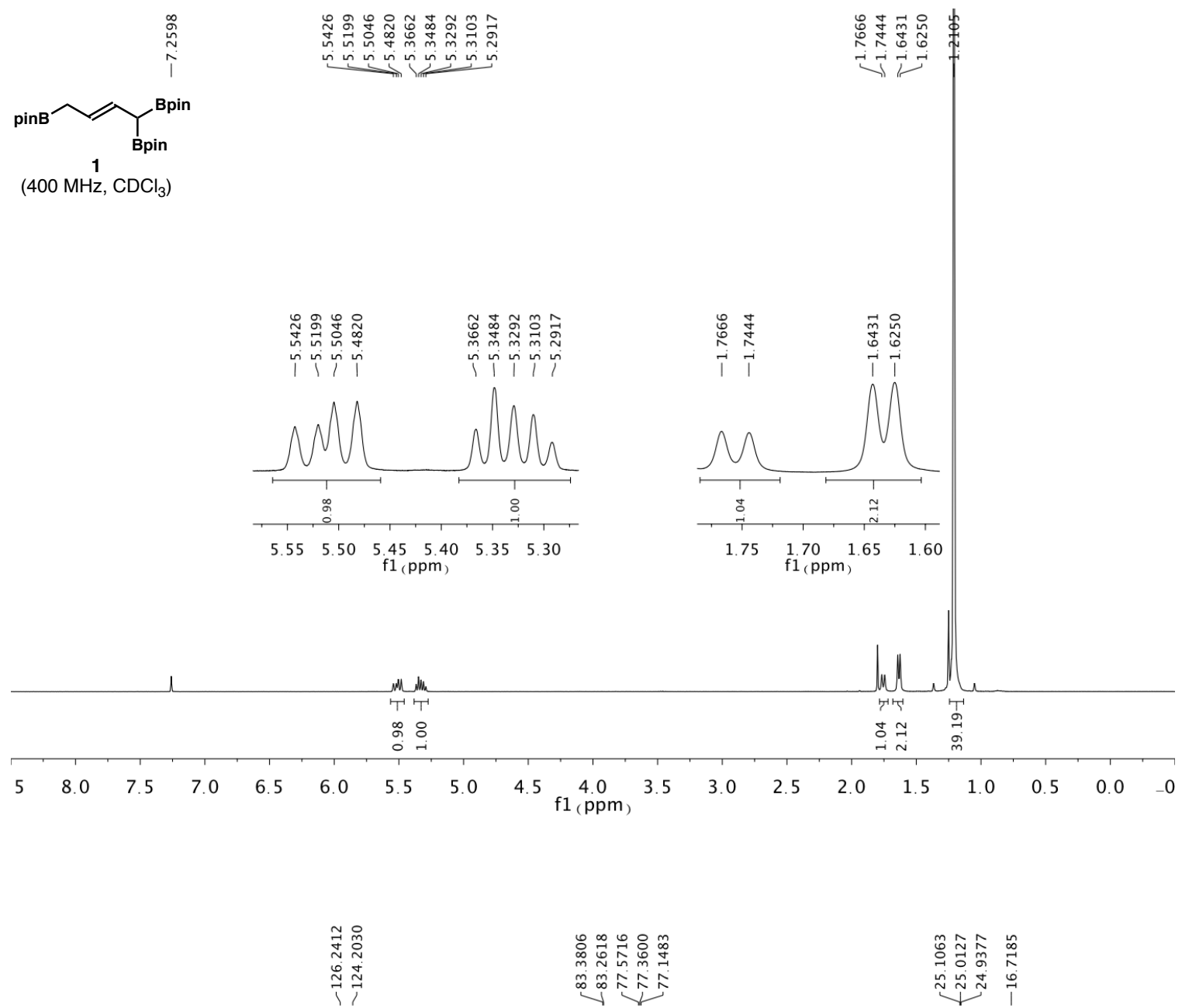

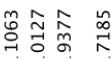

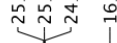

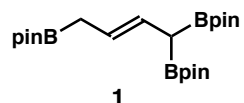

(151 MHz, $\mathrm{CDCl}_{3}$ )

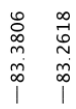

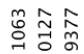

$\stackrel{\sim}{\sim} \stackrel{\sim}{\sim}$

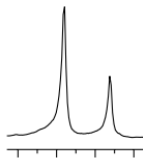

$83.4 \quad 83.2$ f1 (ppm)
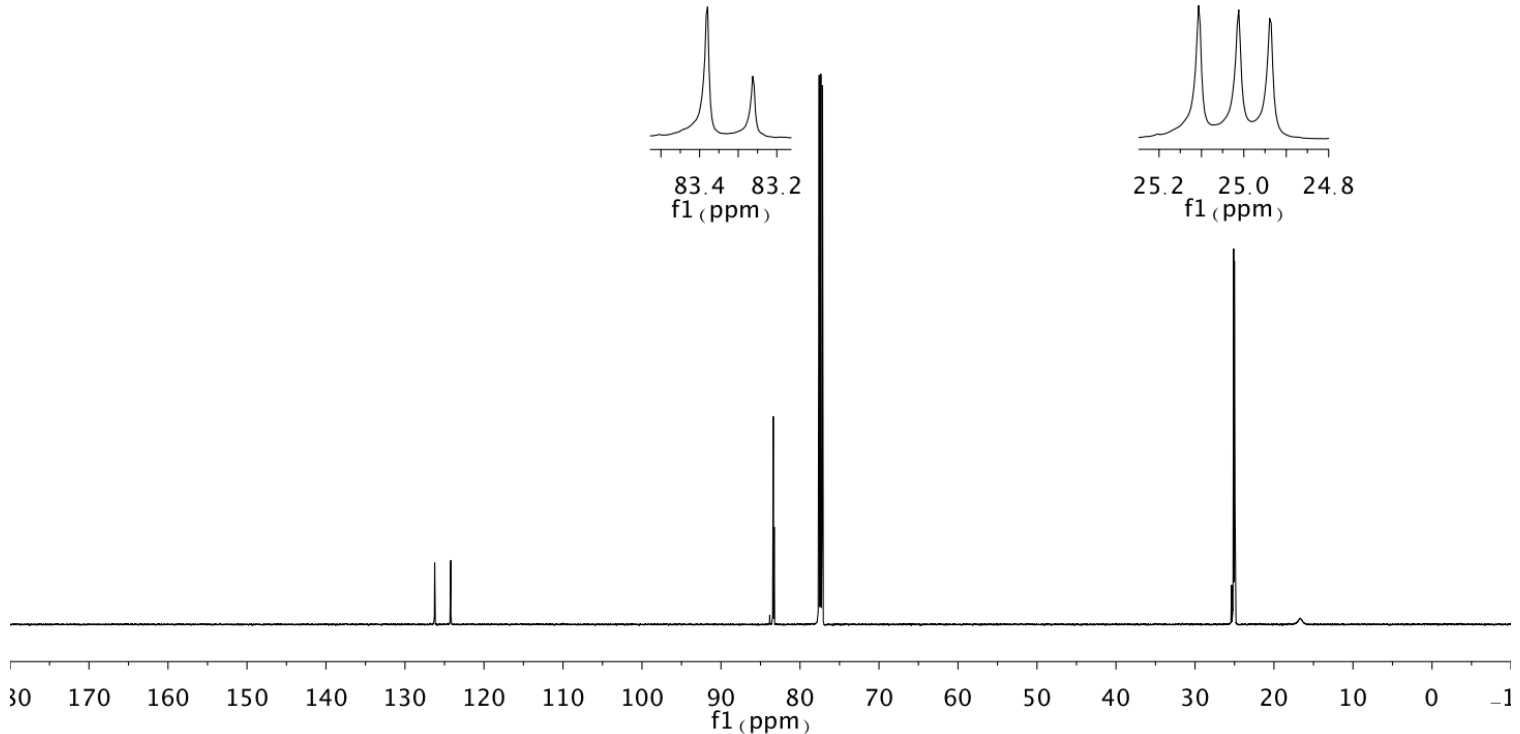

SI-24 

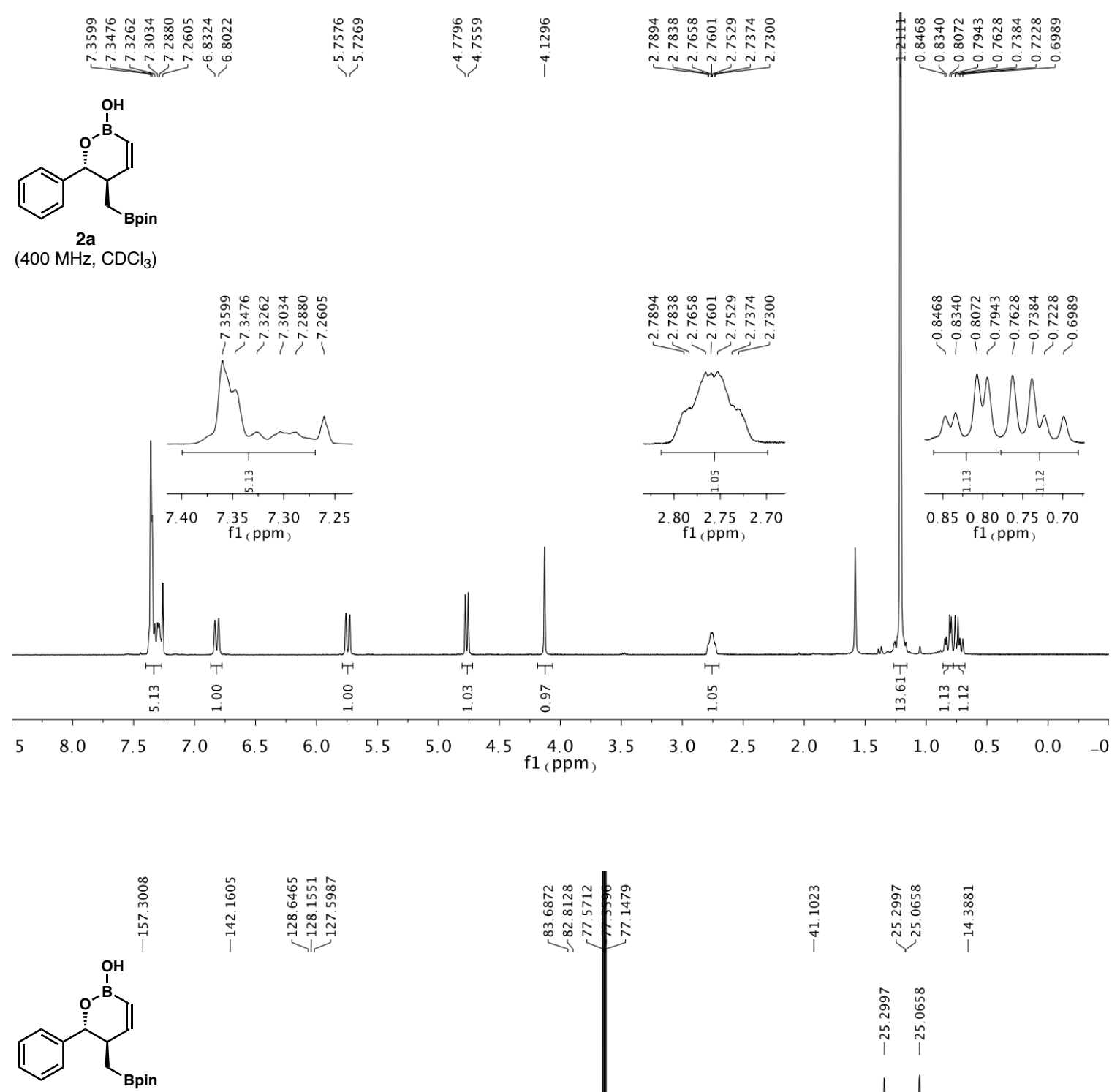

(151 MHz, $\mathrm{CDCl}_{3}$ )

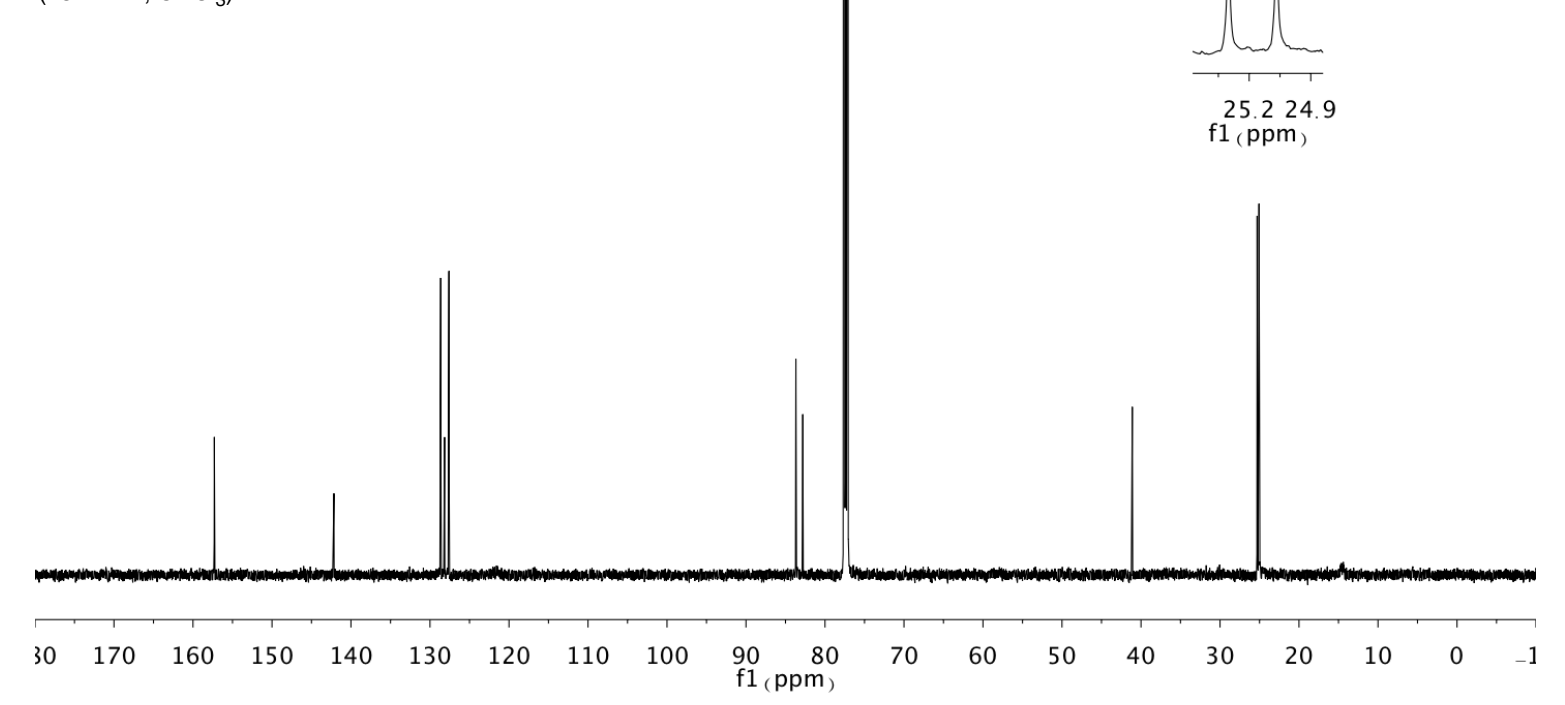



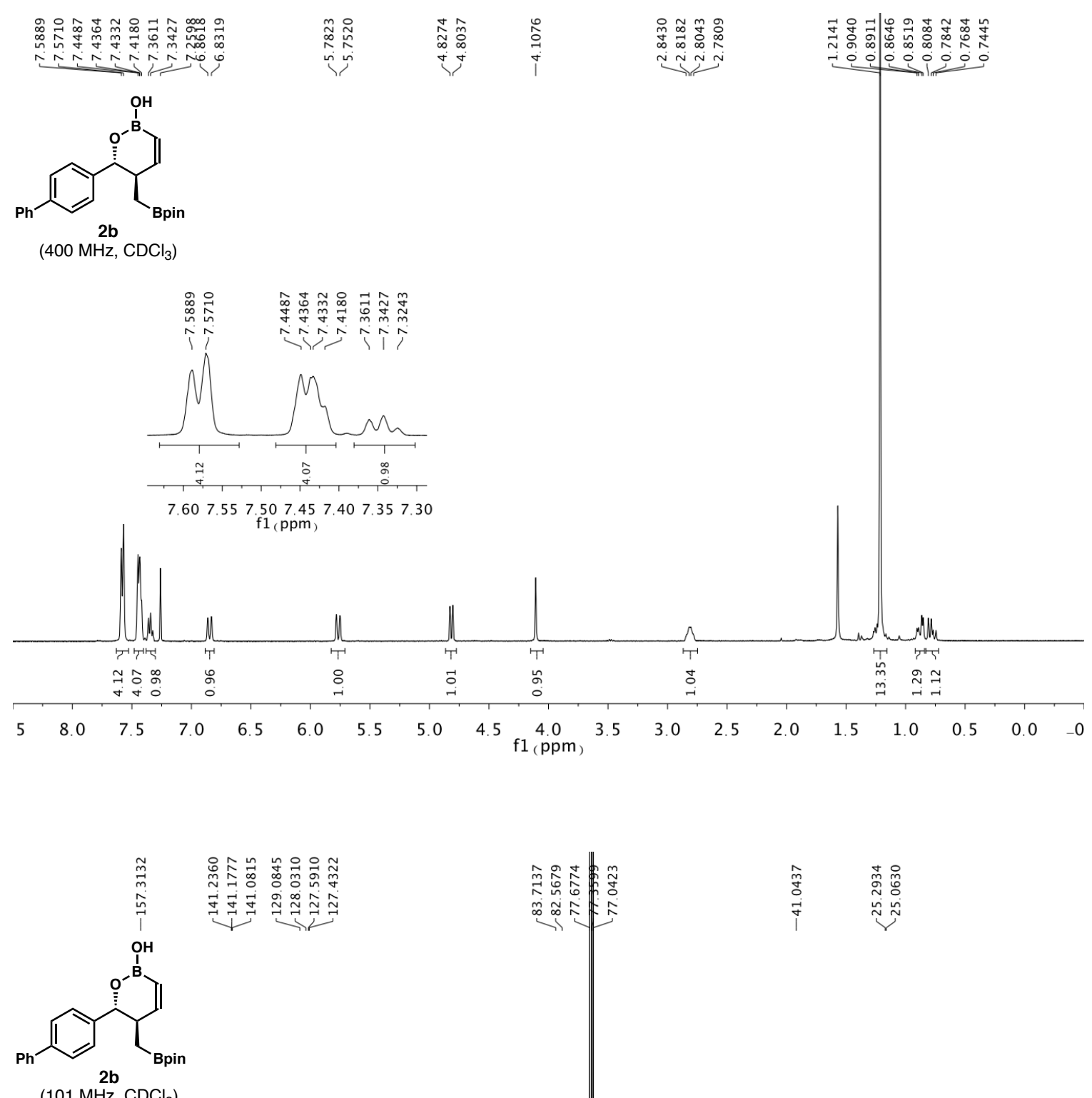

(101 MHz, $\mathrm{CDCl}_{3}$ )

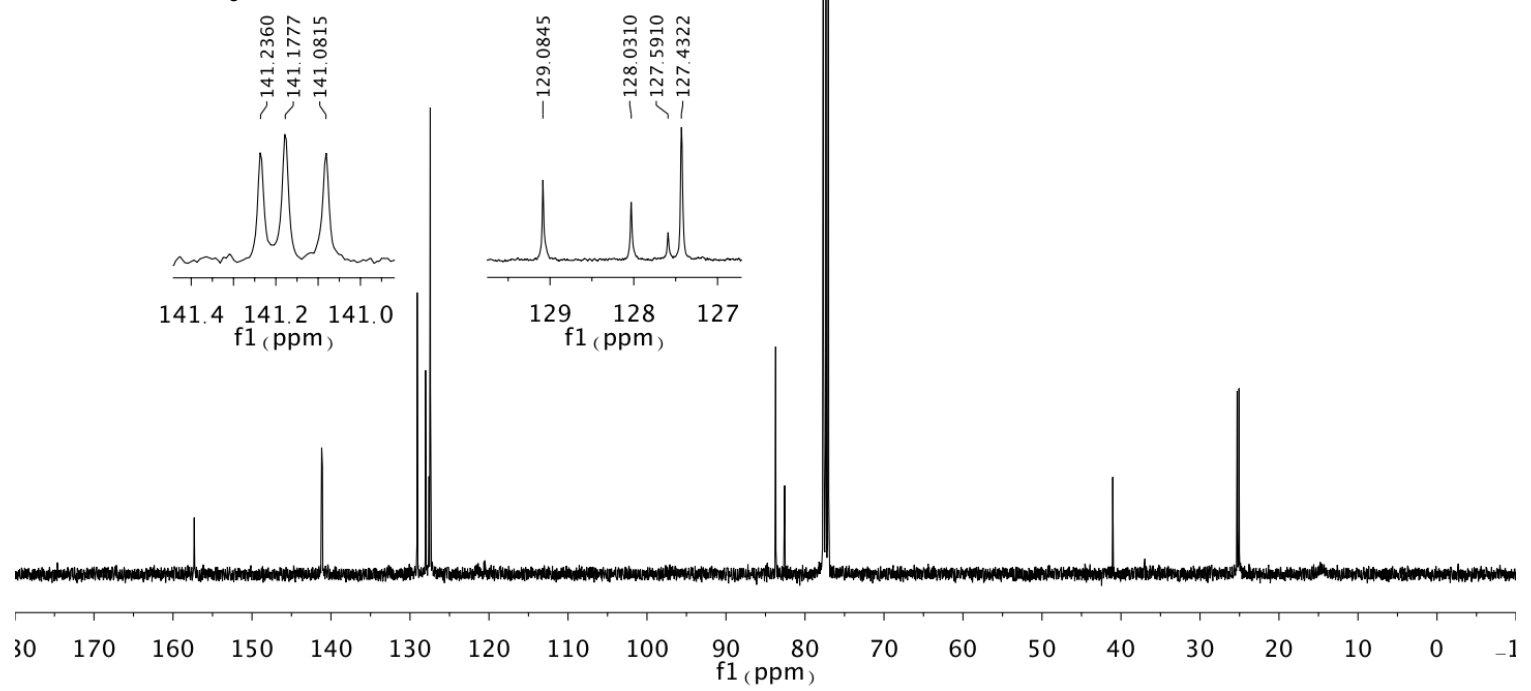



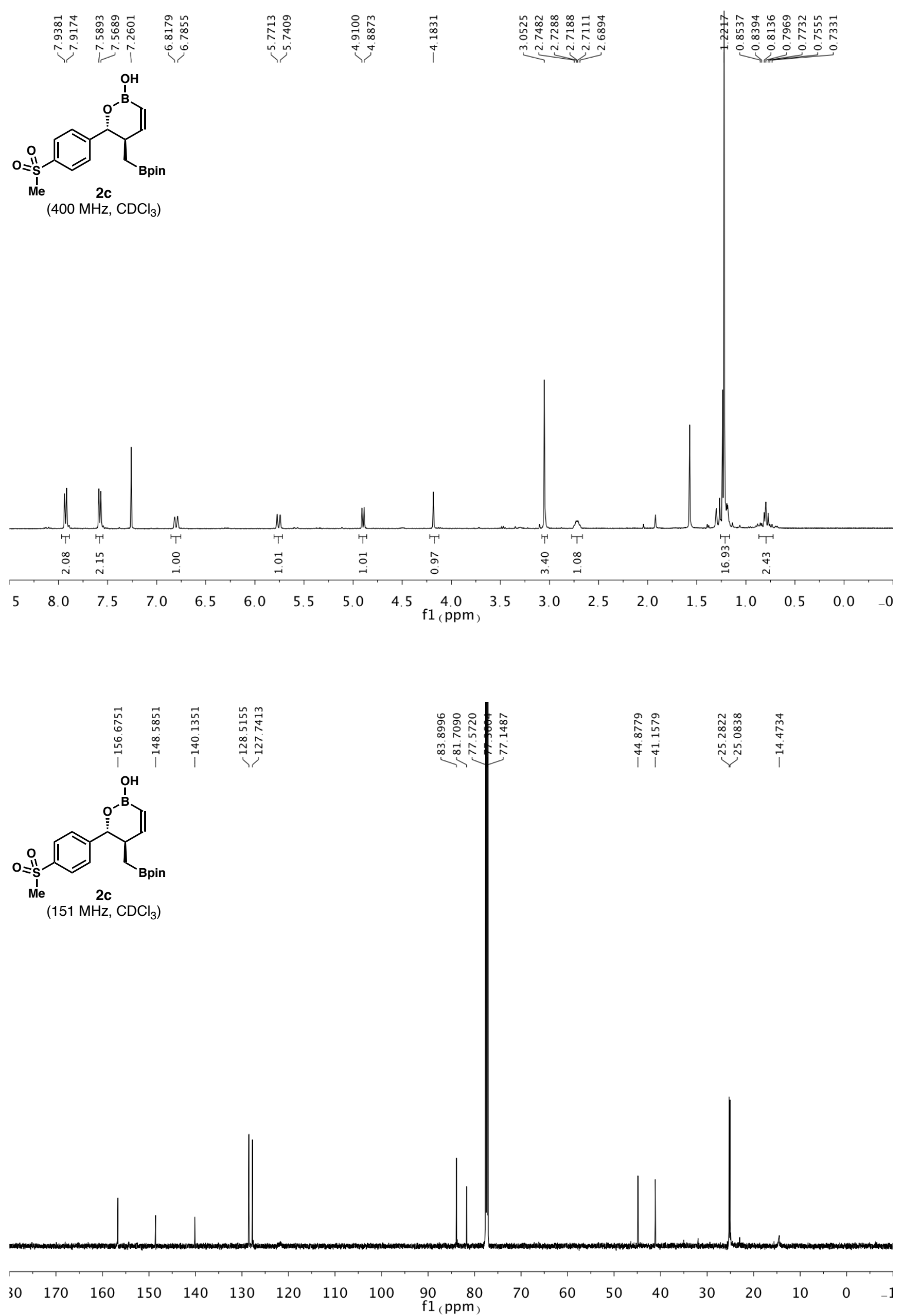

SI-27 

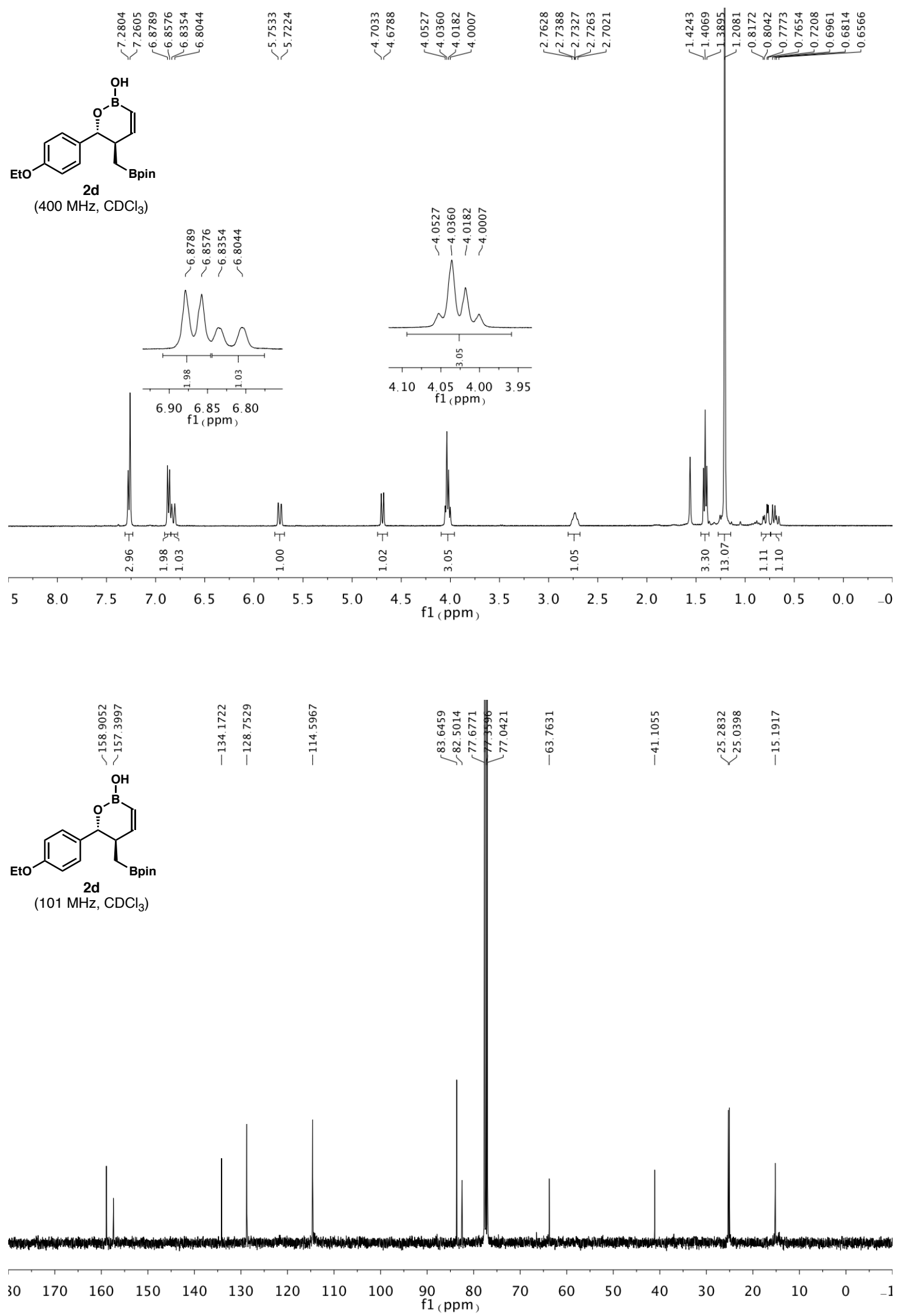

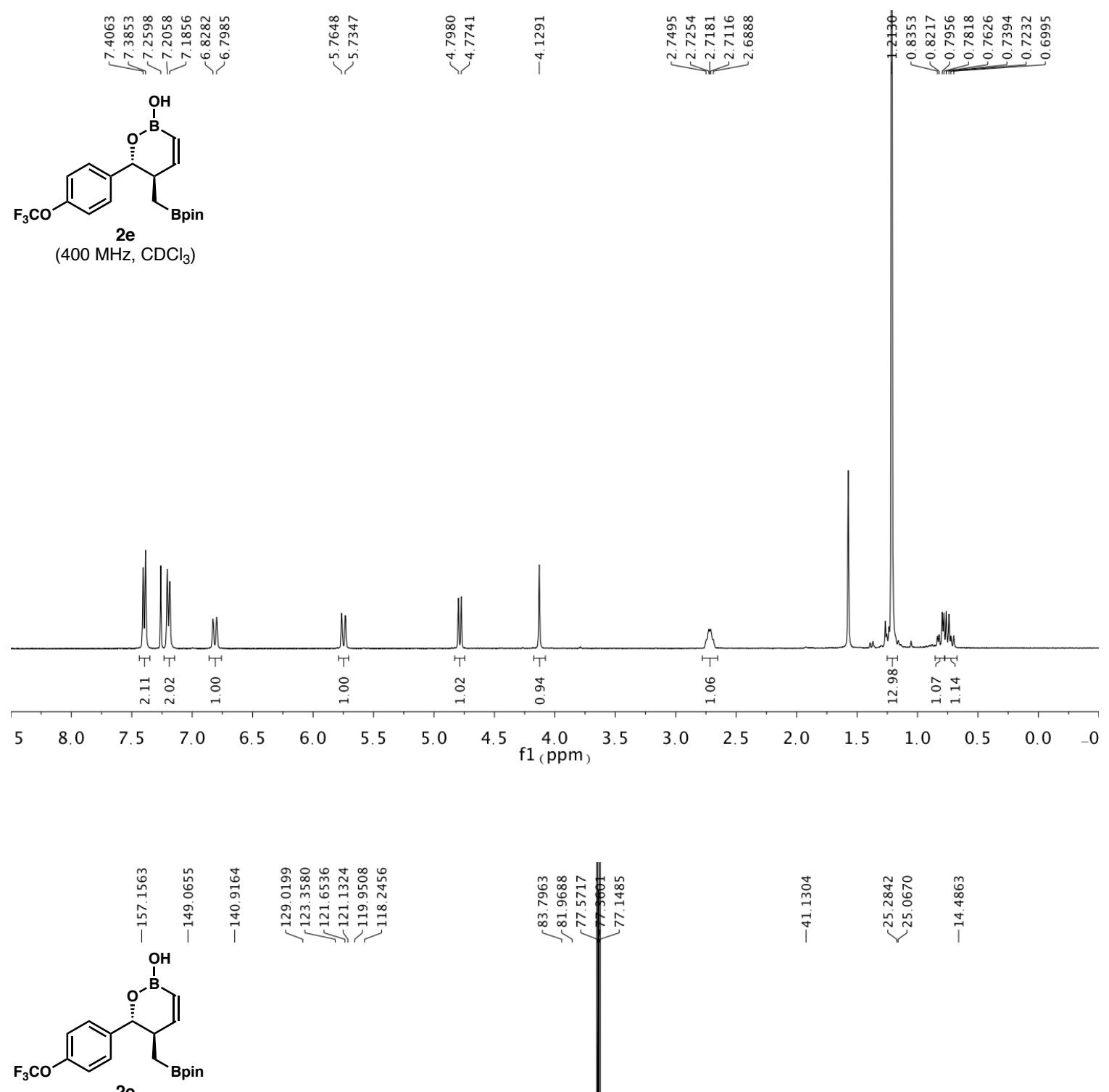

$\left(151 \mathrm{MHz}, \mathrm{CDCl}_{3}\right.$ )

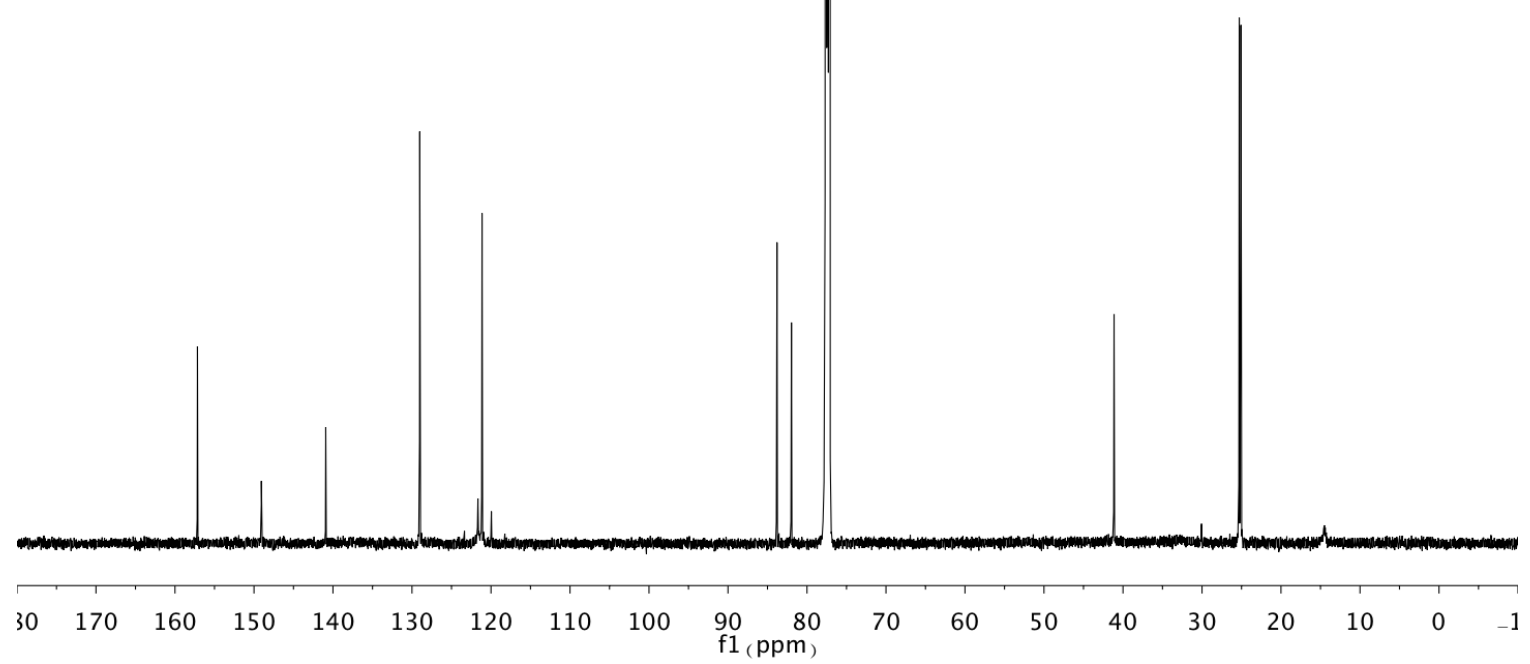

SI-29 

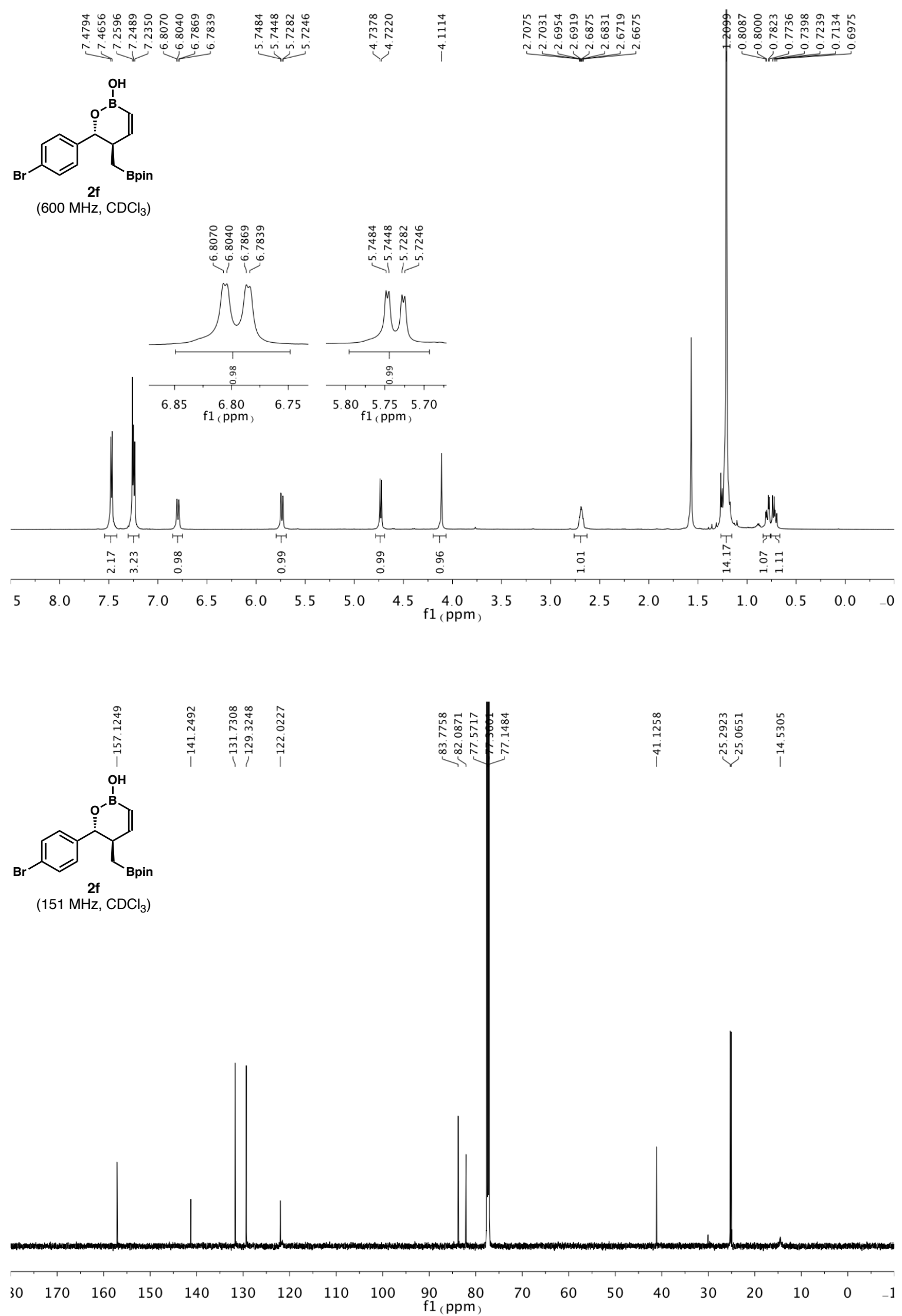

SI-30 

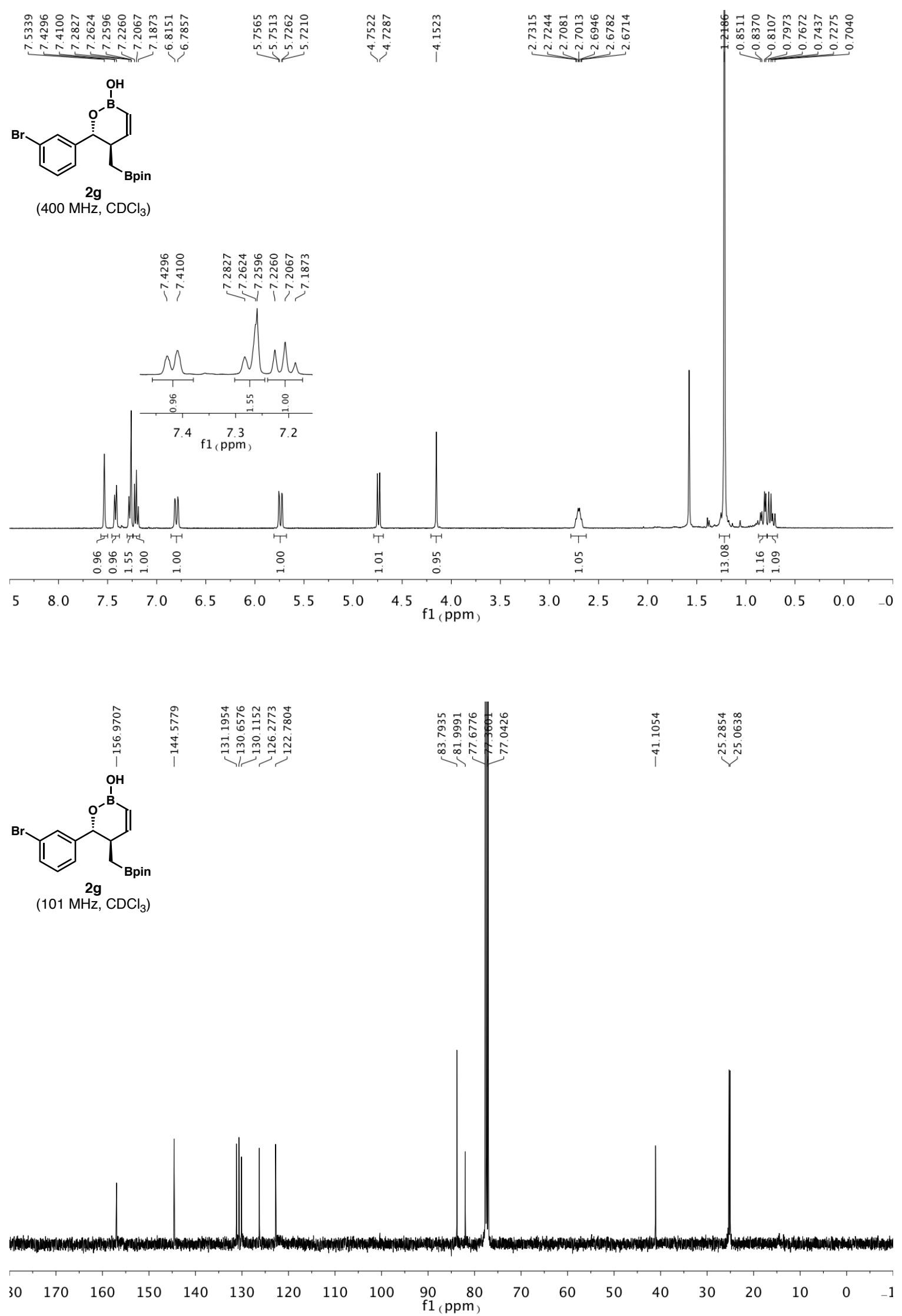

SI-31 


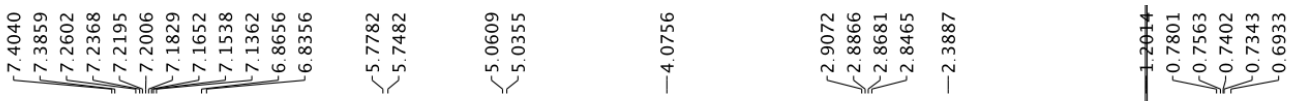

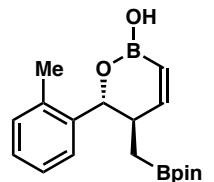

2h

$\left(400 \mathrm{MHz}, \mathrm{CDCl}_{3}\right)$
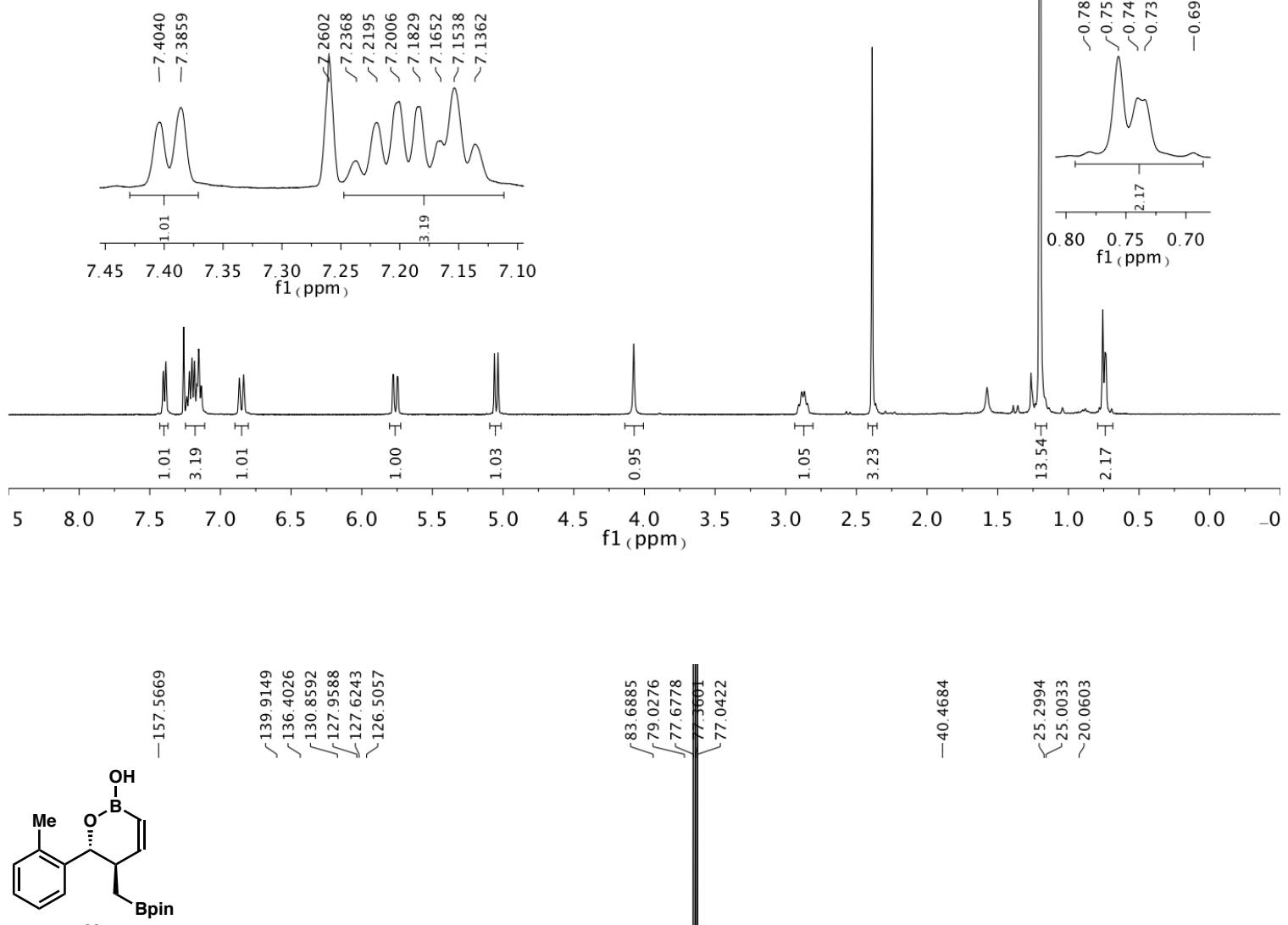

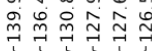
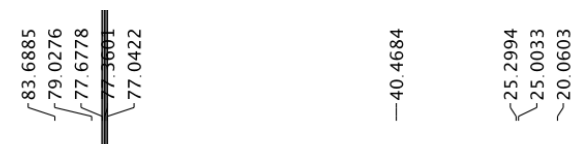

$\left(101 \mathrm{MHz}, \mathrm{CDCl}_{3}\right)$
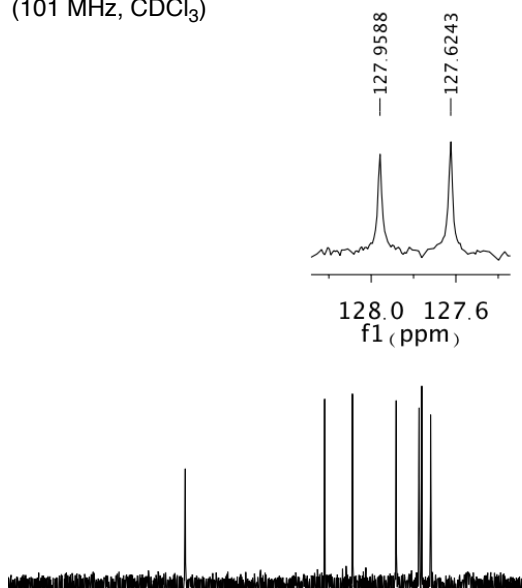

30

$150 \quad 140$

130

$20 \quad 110 \quad 100$

$90 \quad 80$

㕝总总策

íi,i i

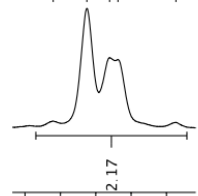

$\begin{array}{lll}0.80 & 0.75 & 0.70\end{array}$

$\mathrm{f} 1(\mathrm{ppm})$ 

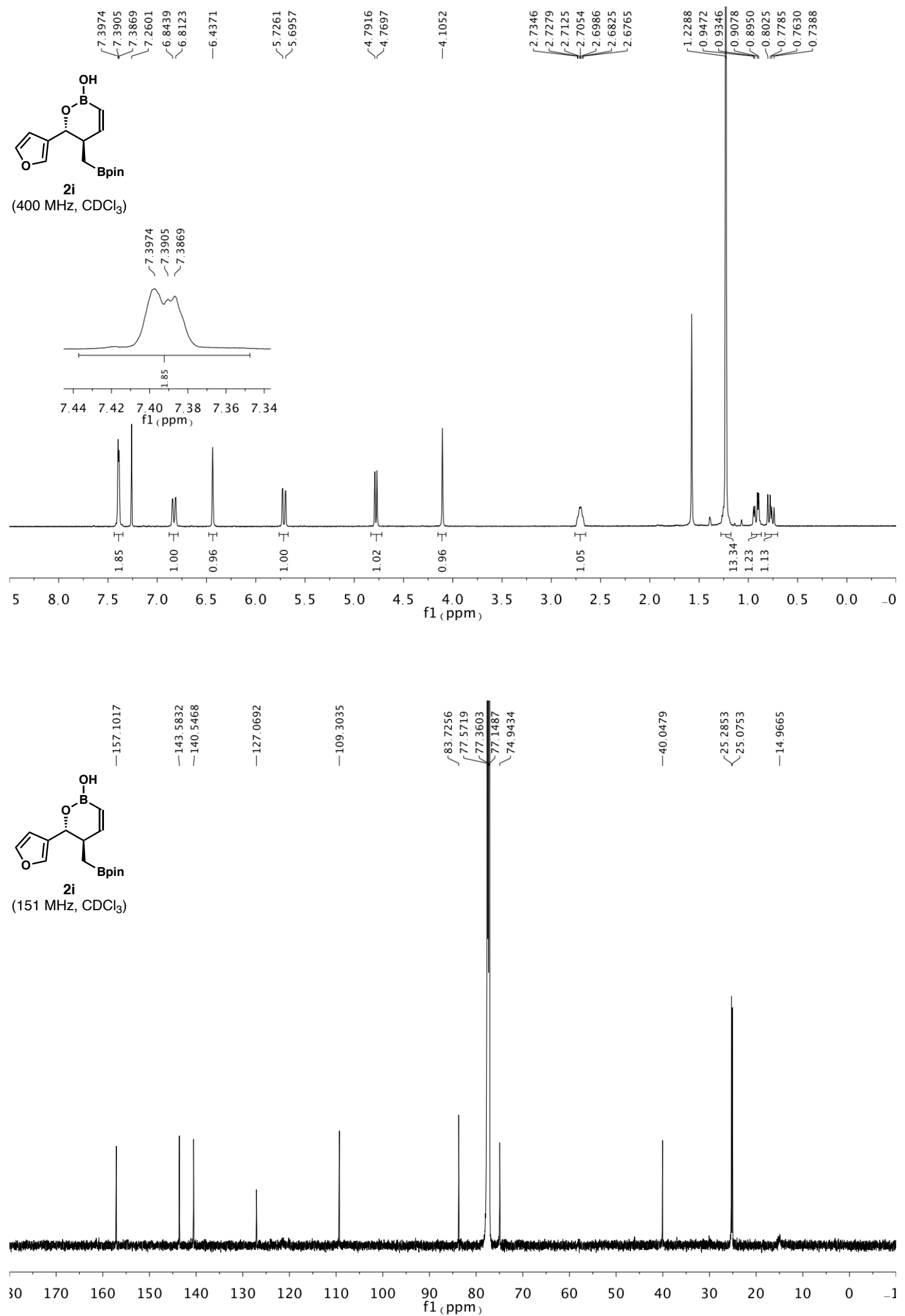

SI-33 

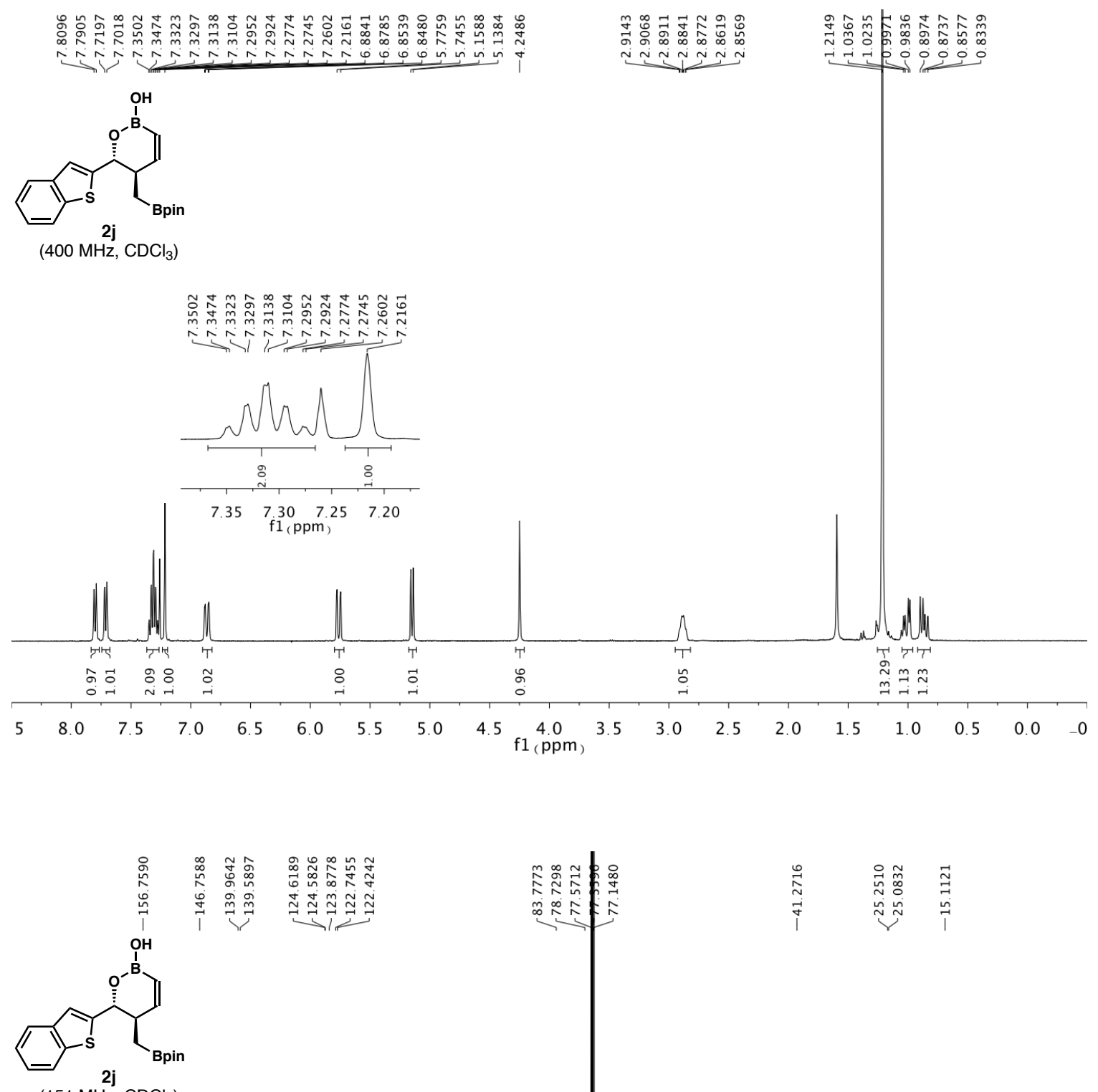

(151 MHz, $\mathrm{CDCl}_{3}$ )

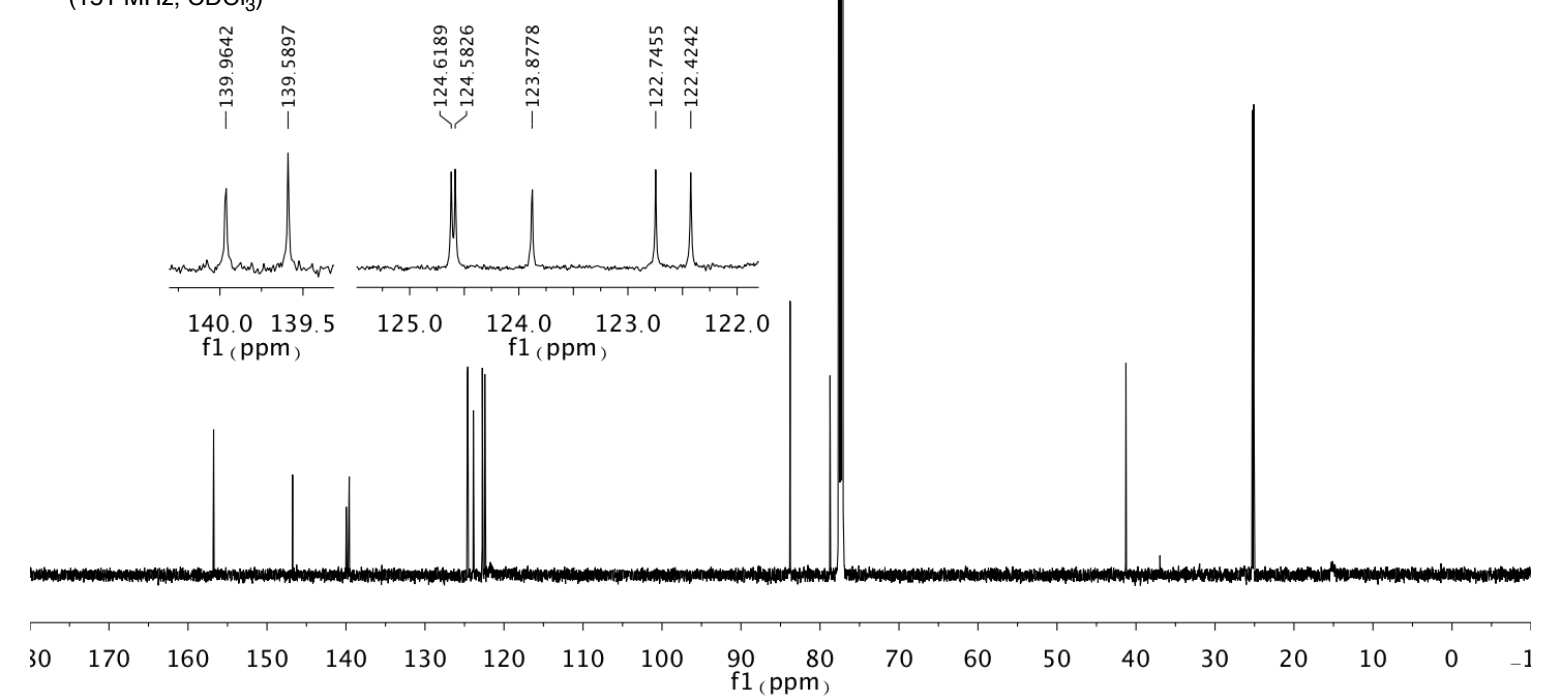

SI-34 

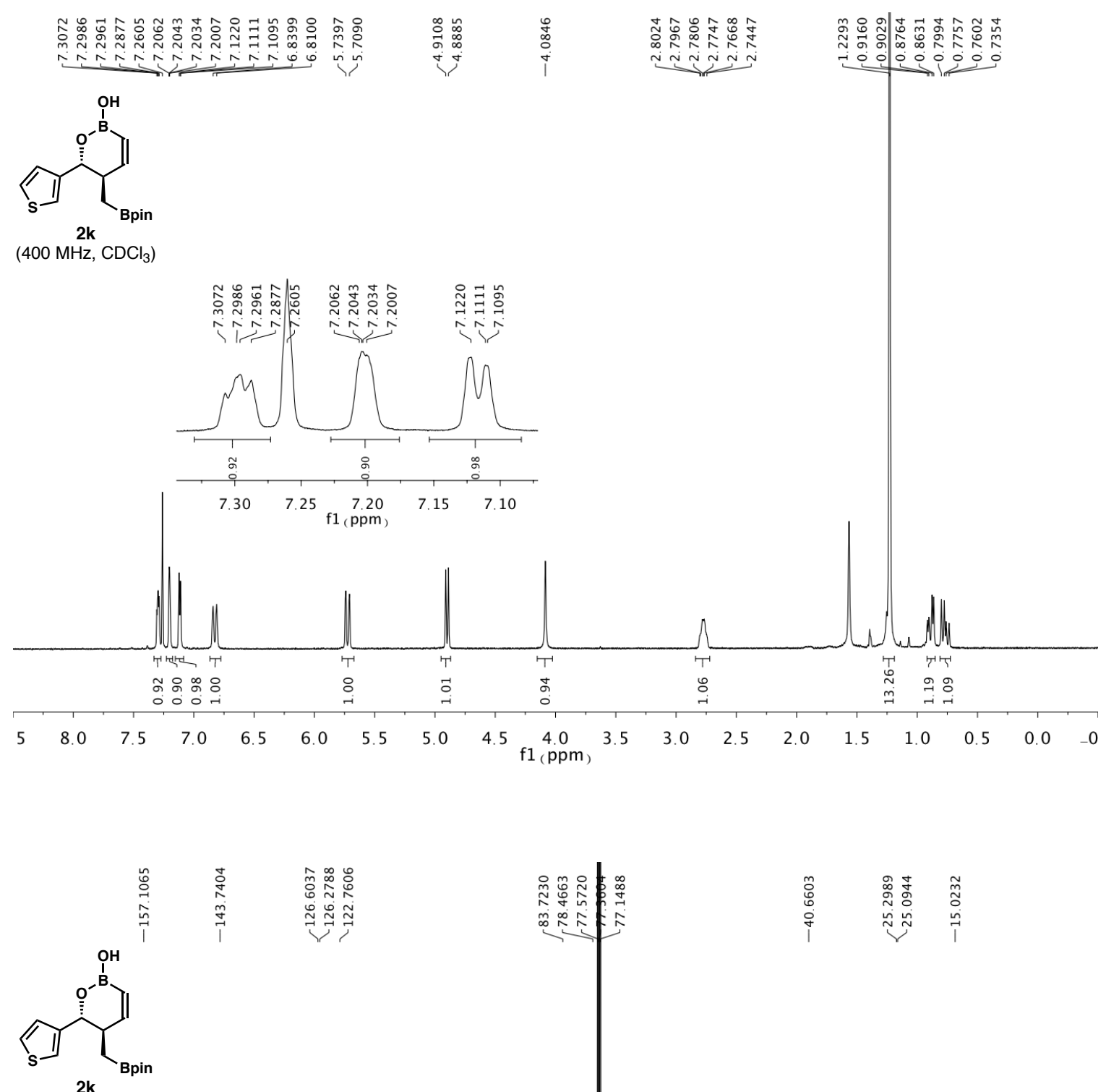

(151 MHz, $\mathrm{CDCl}_{3}$ )
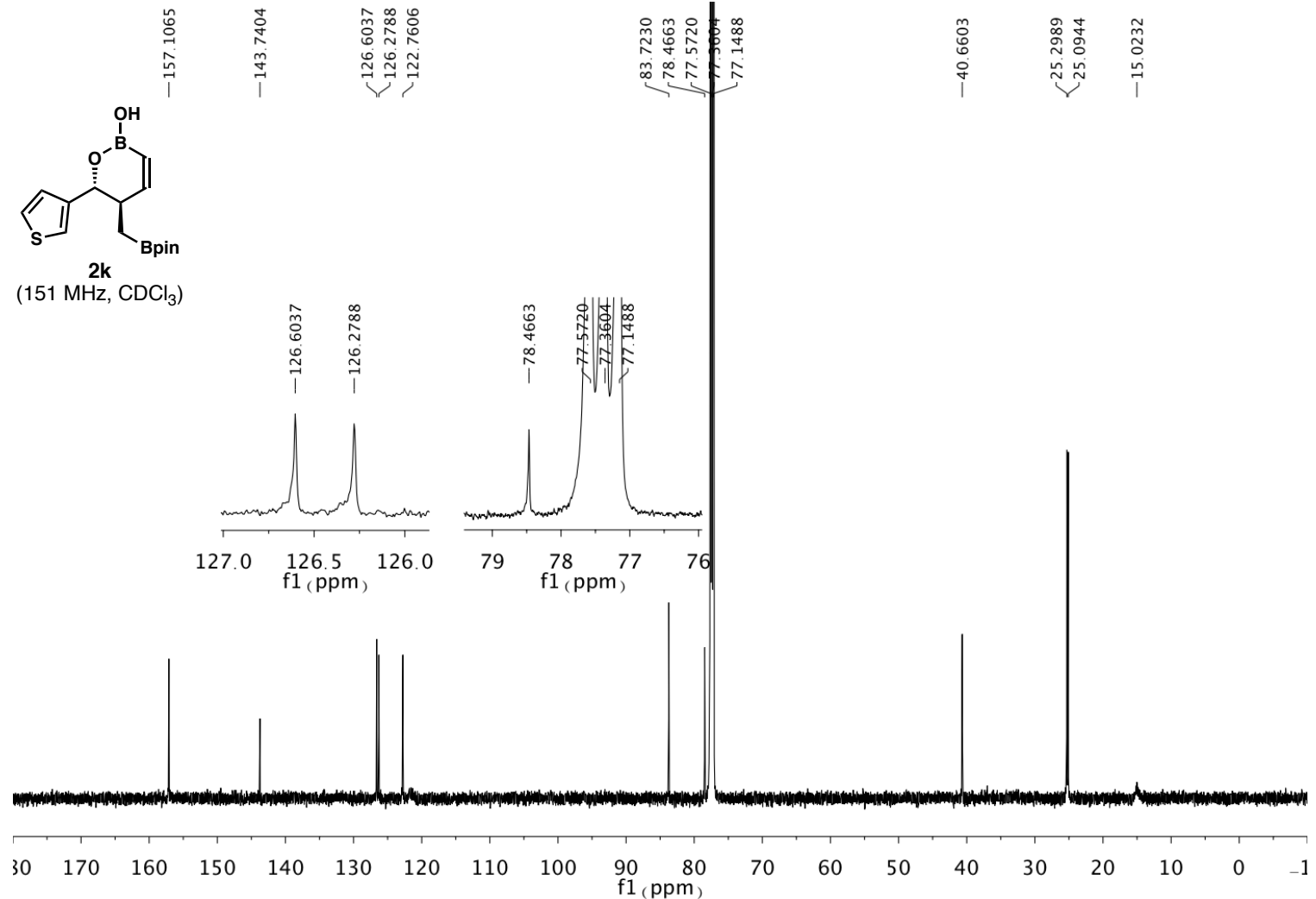

SI-35 

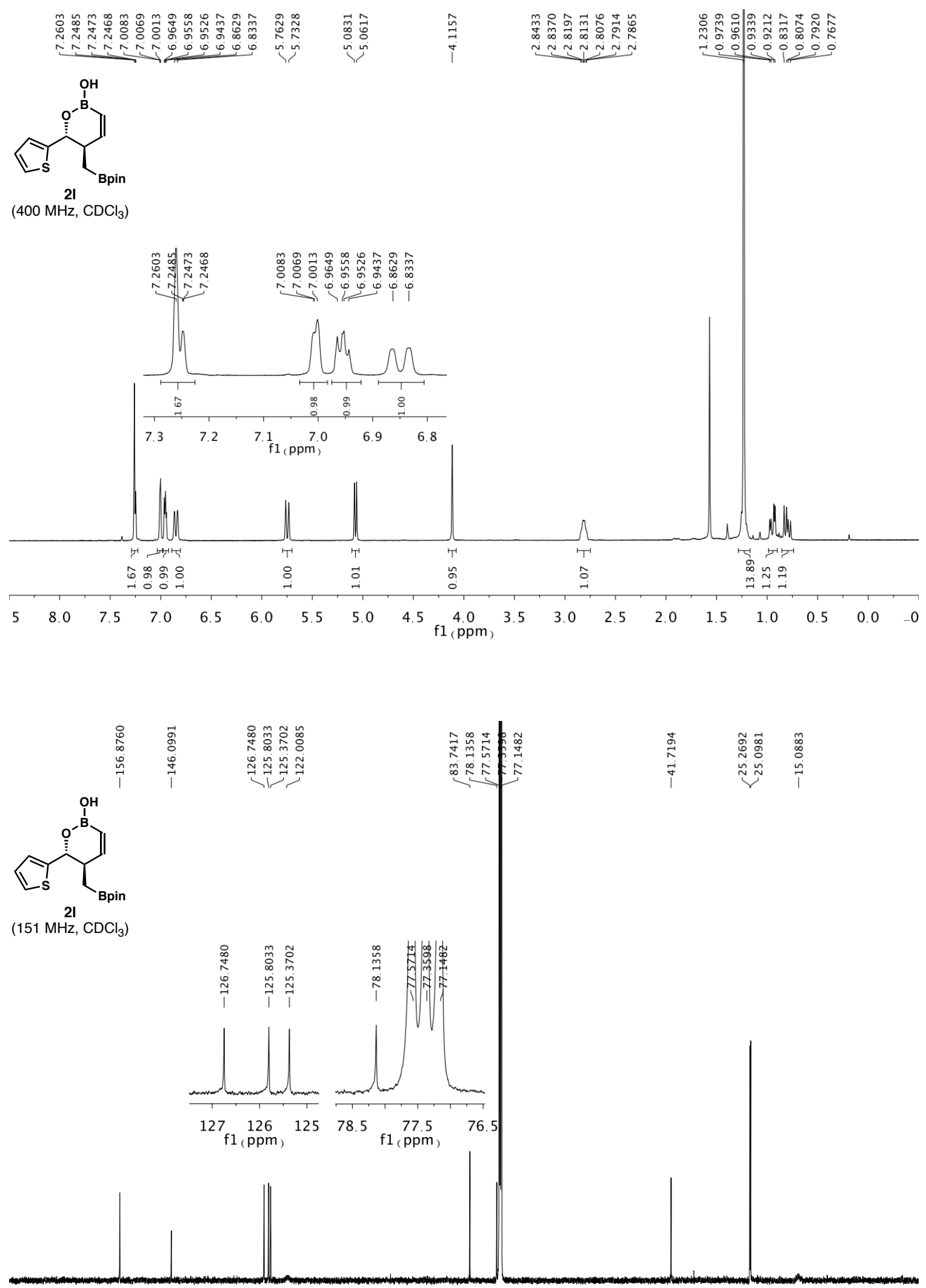

$\begin{array}{lllllllllll}30 & 170 & 160 & 150 & 140 & 130 & 120 & 110 & 100 & 90 & 80\end{array}$

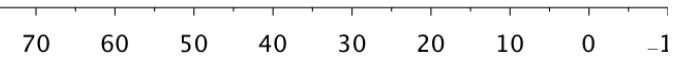



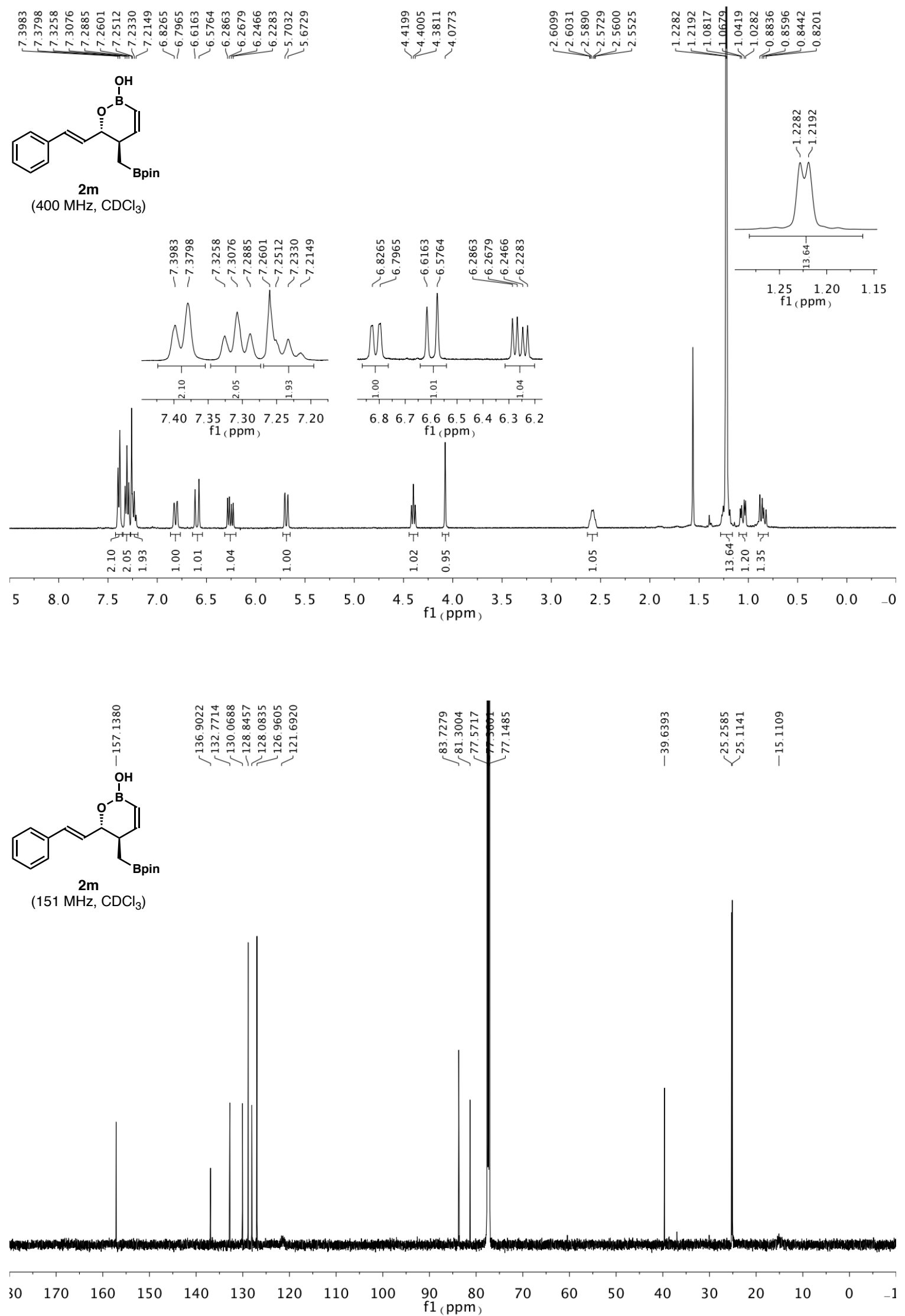

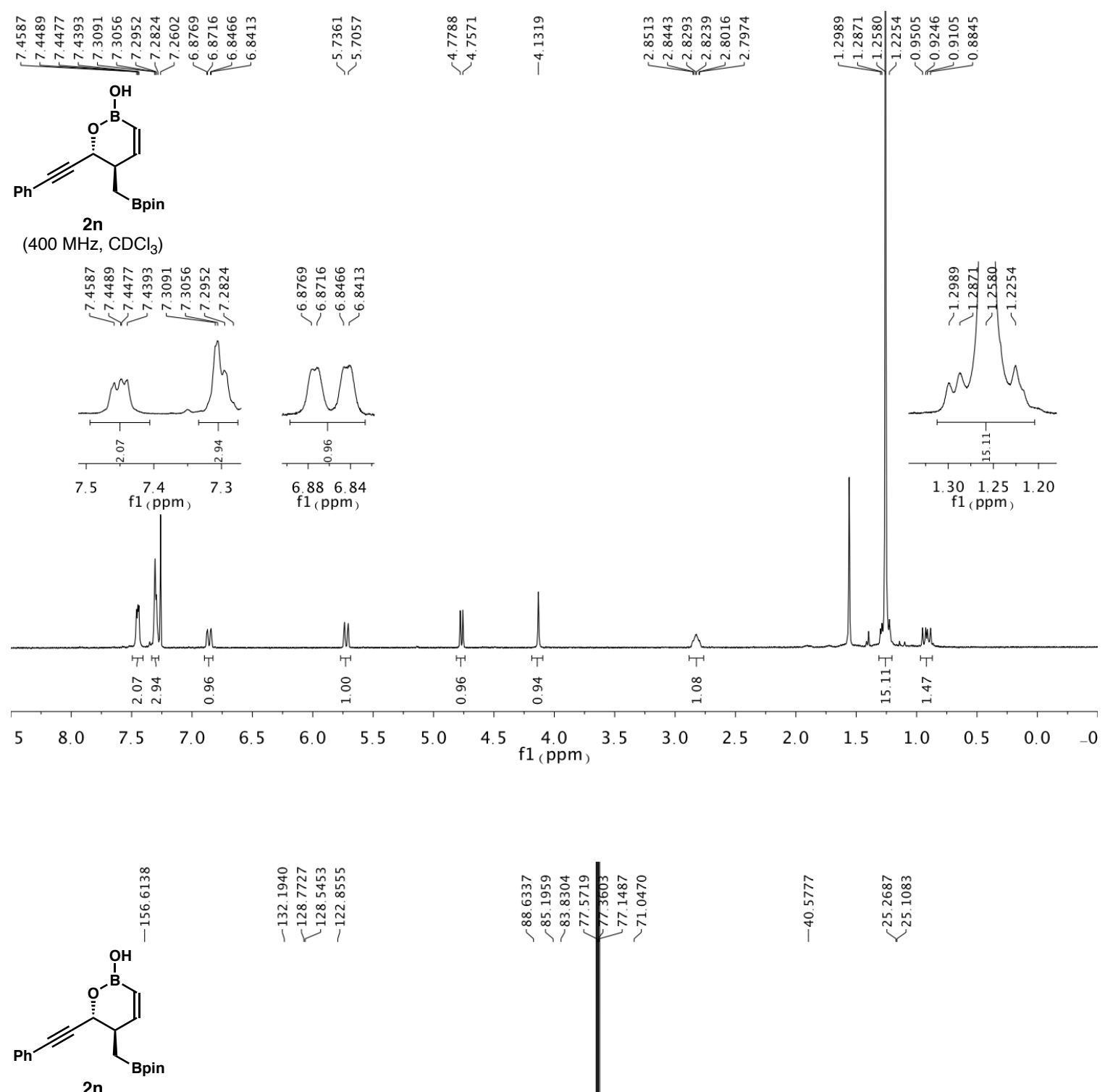

(151 MHz, $\mathrm{CDCl}_{3}$ )

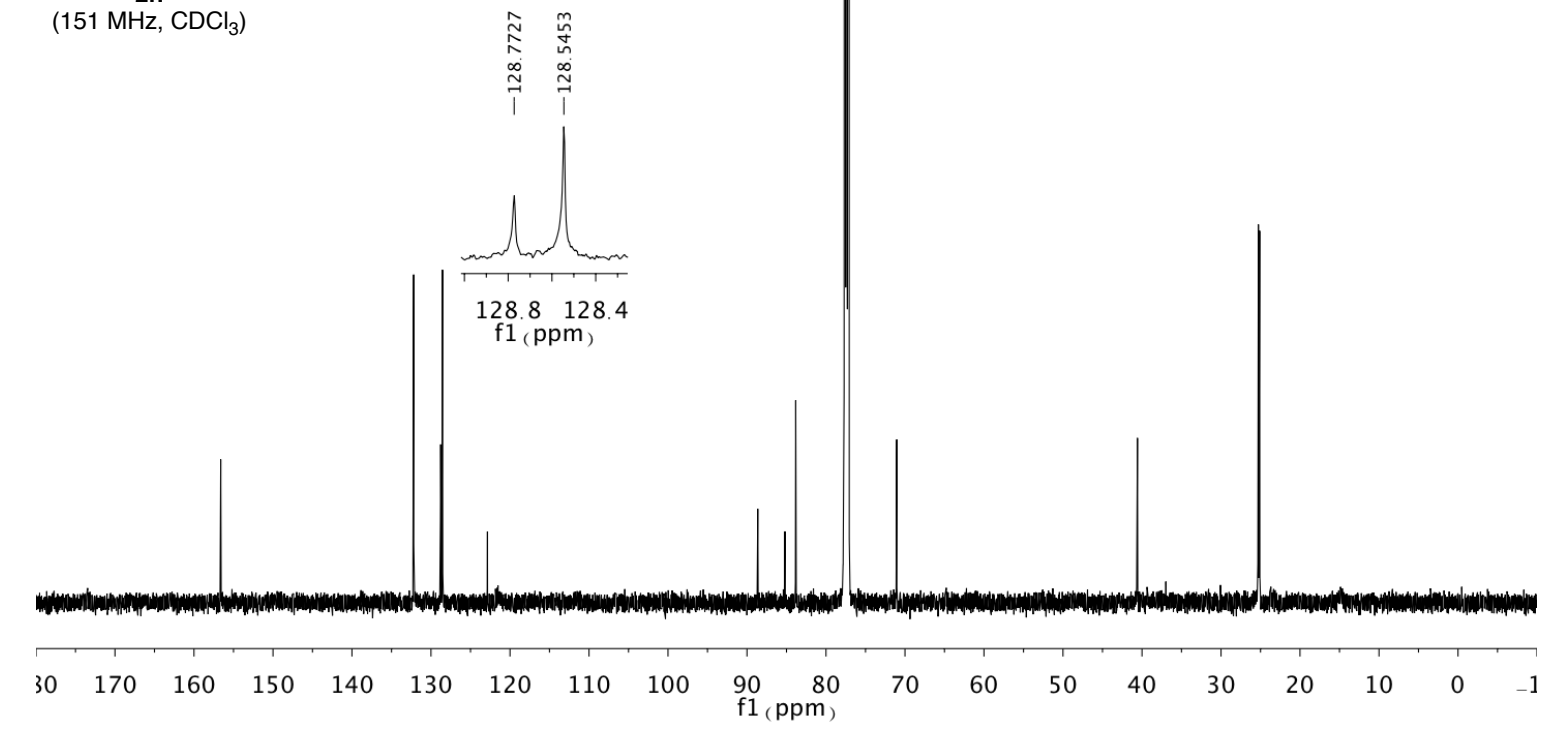

SI-38 

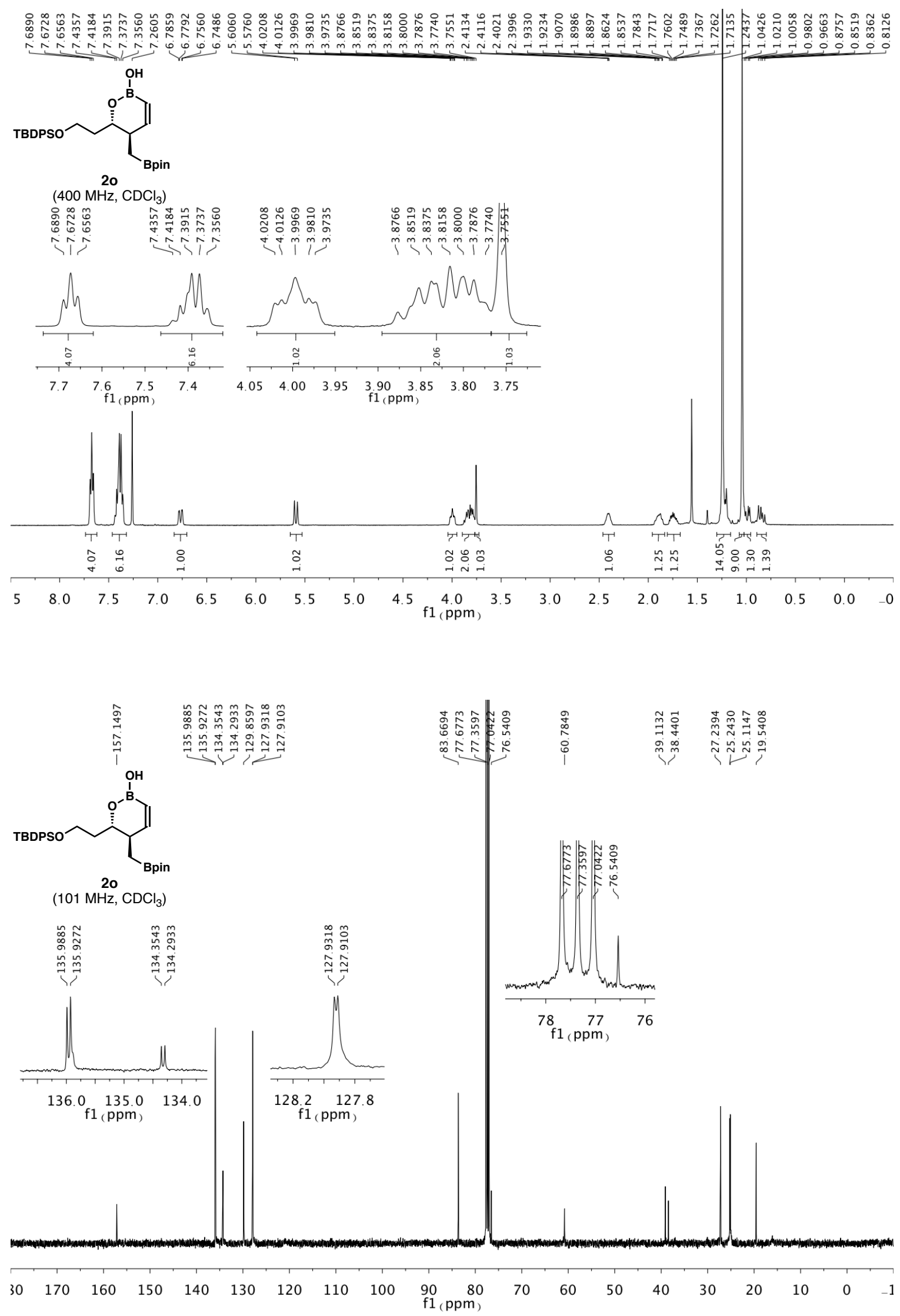

SI-39 

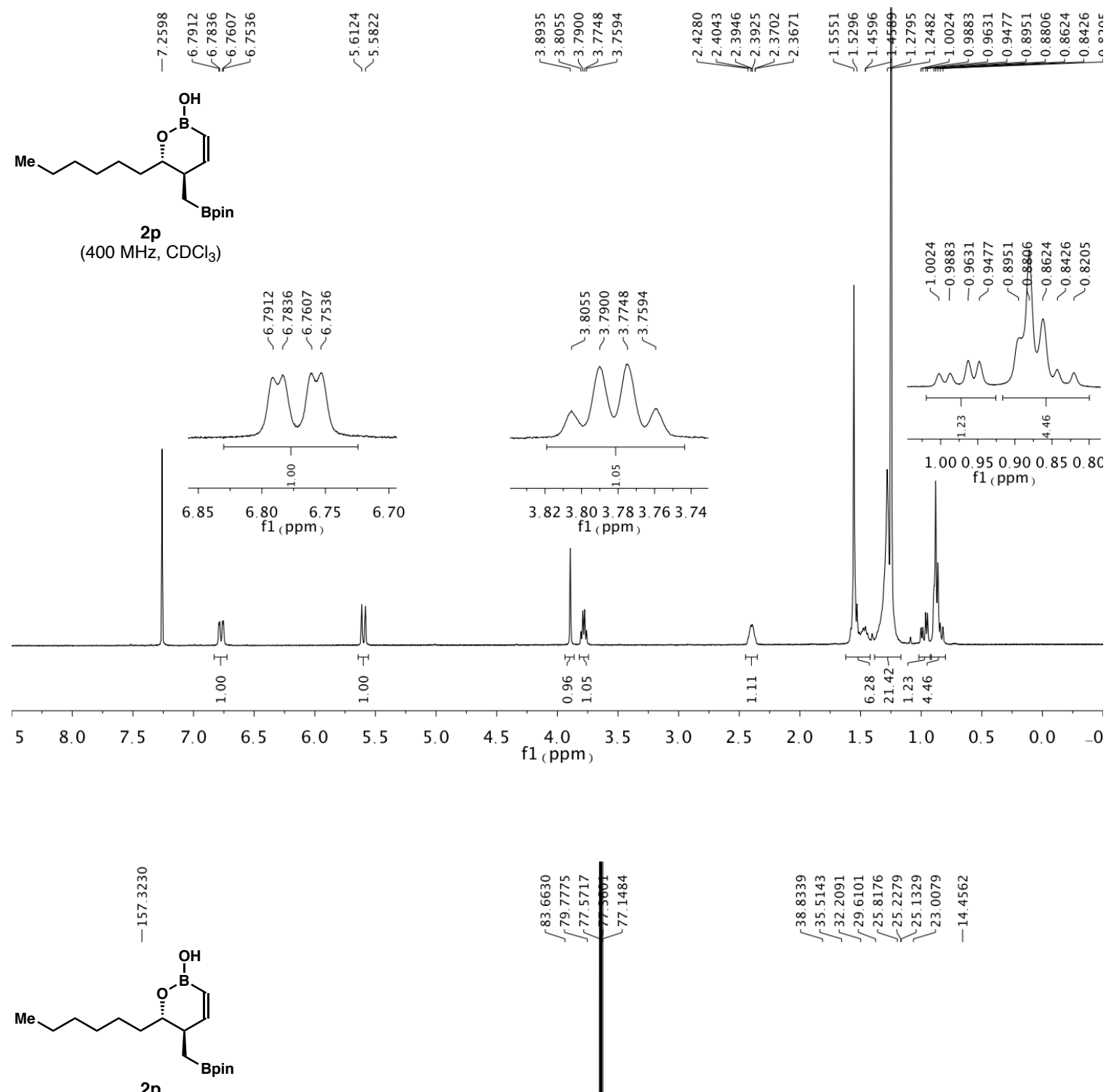

(151 MHz, $\mathrm{CDCl}_{3}$ )

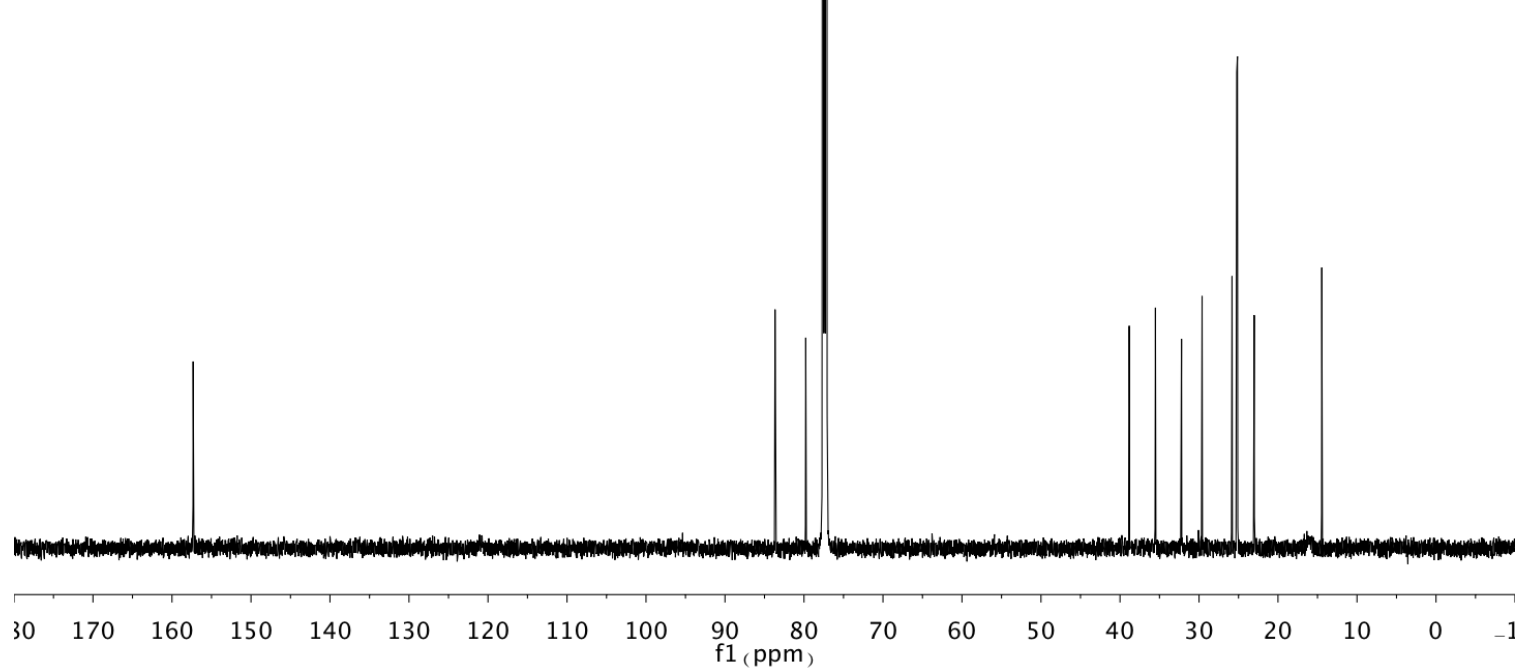



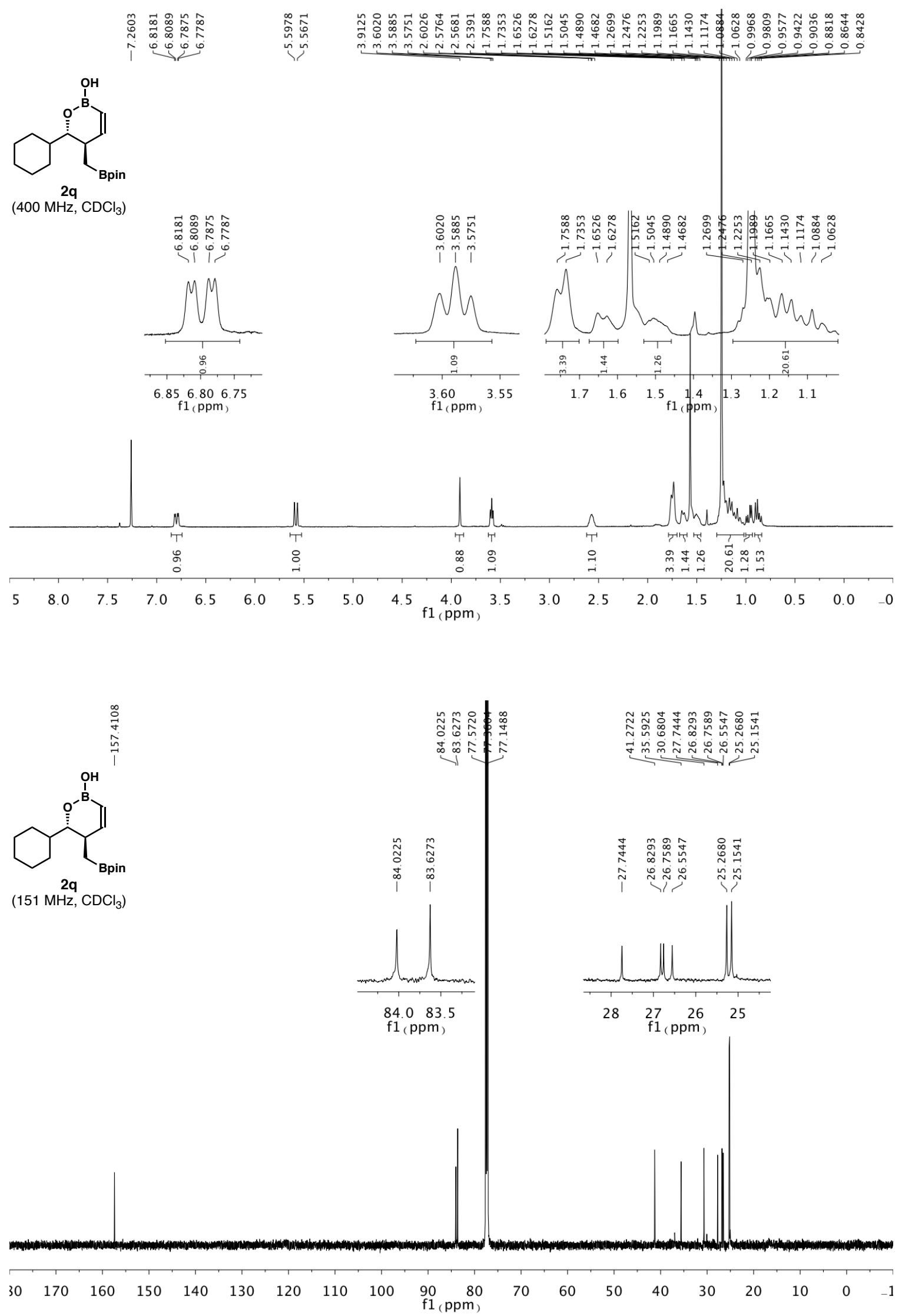

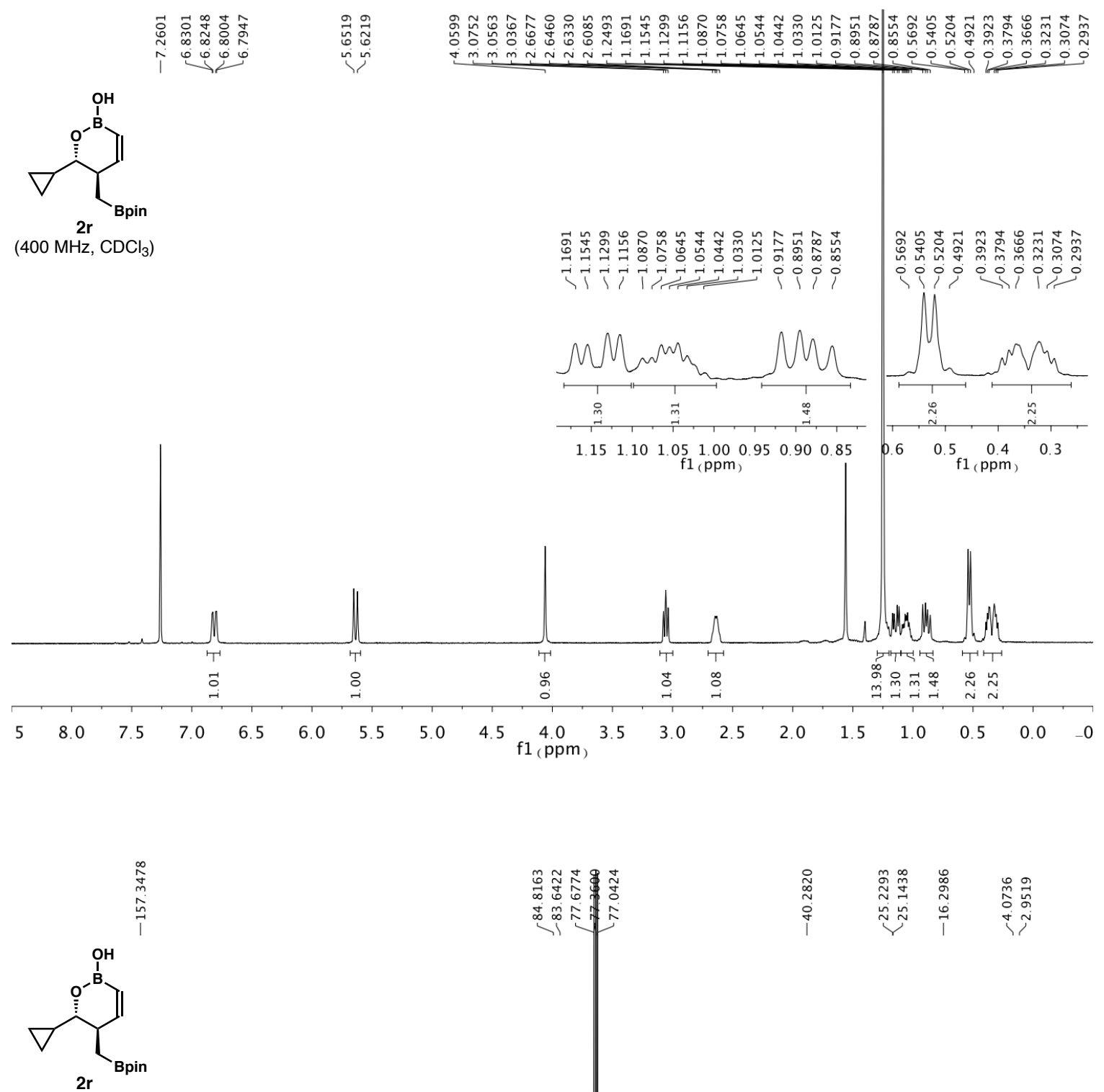

$\left(101 \mathrm{MHz}, \mathrm{CDCl}_{3}\right.$ )

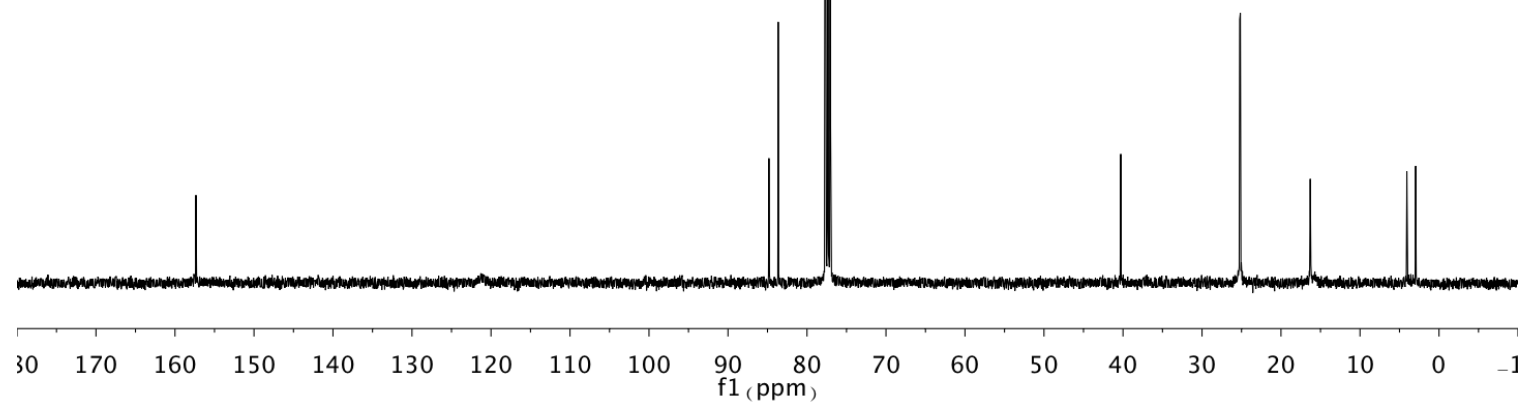



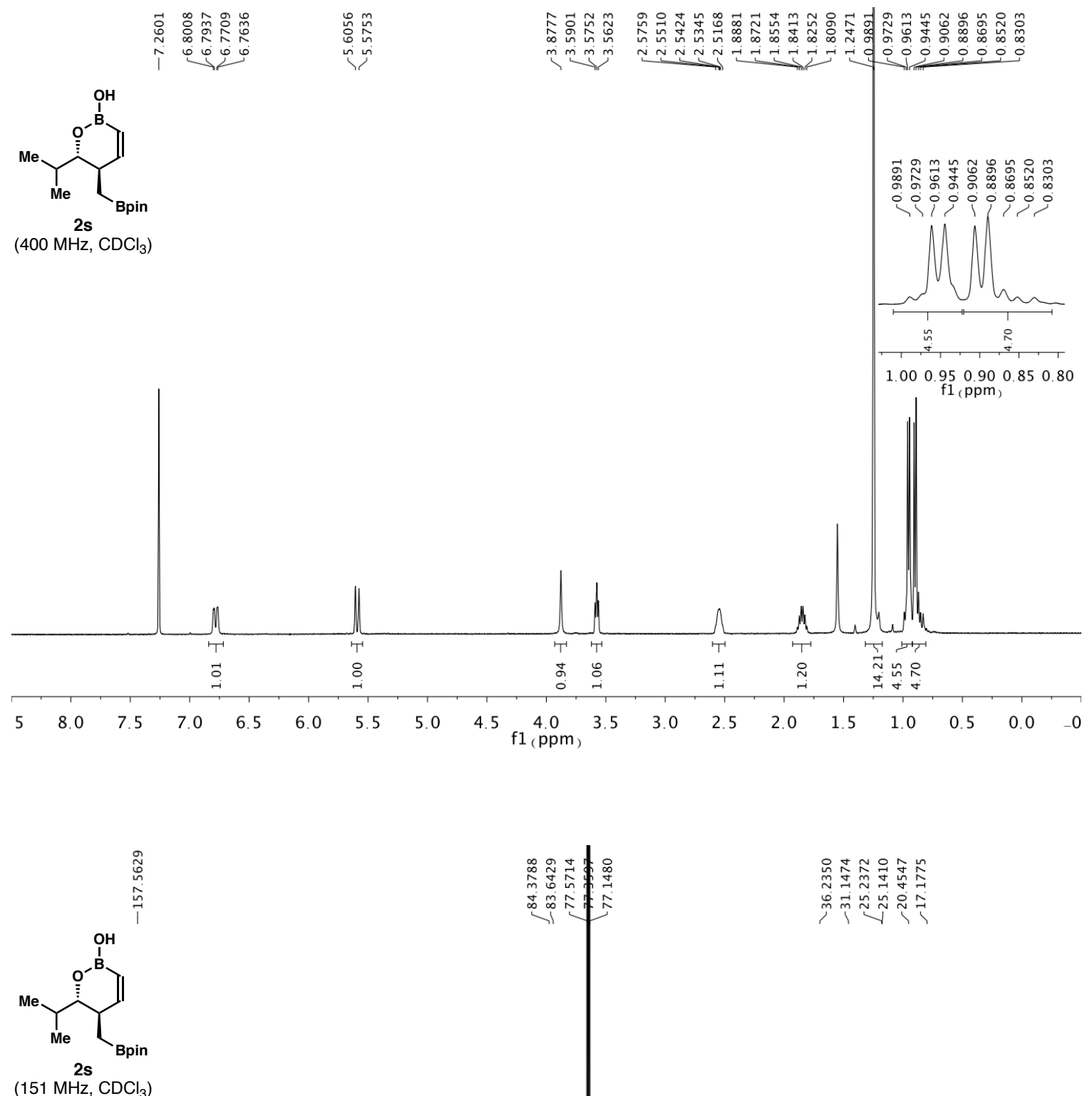

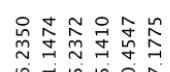

ํำกำ ำ

(151 MHz, $\mathrm{CDCl}_{3}$ )

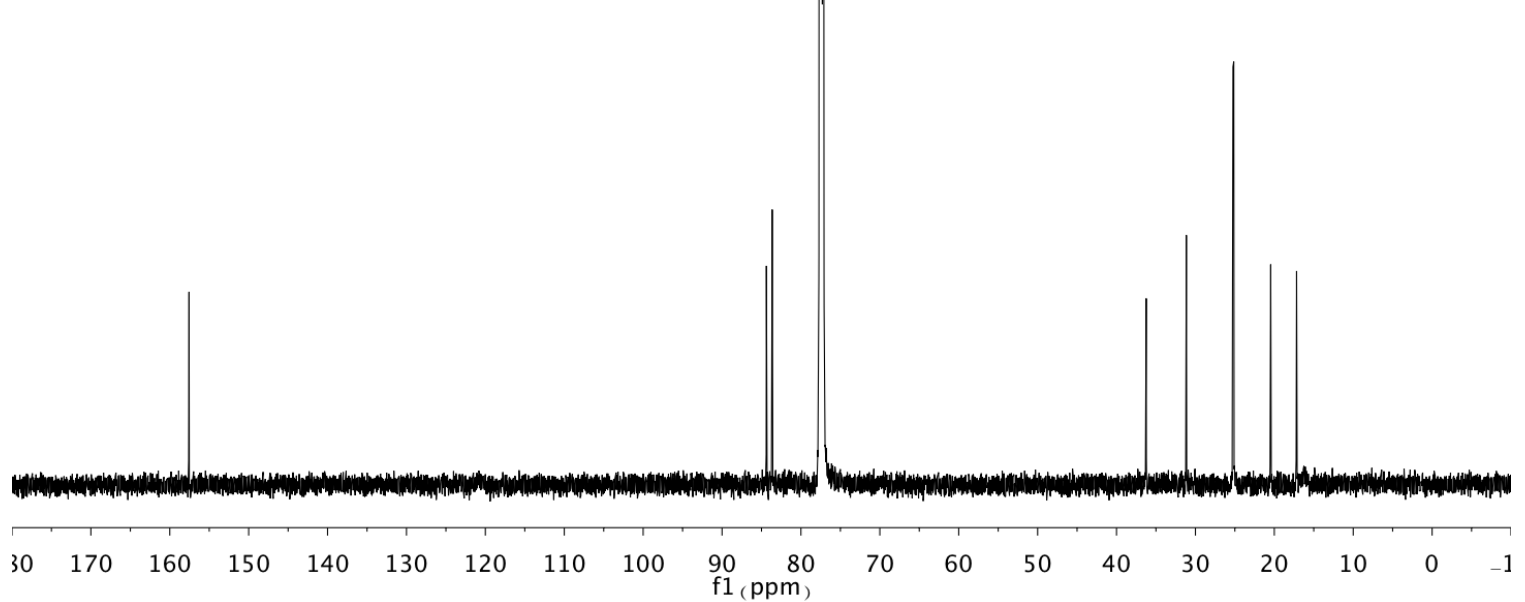



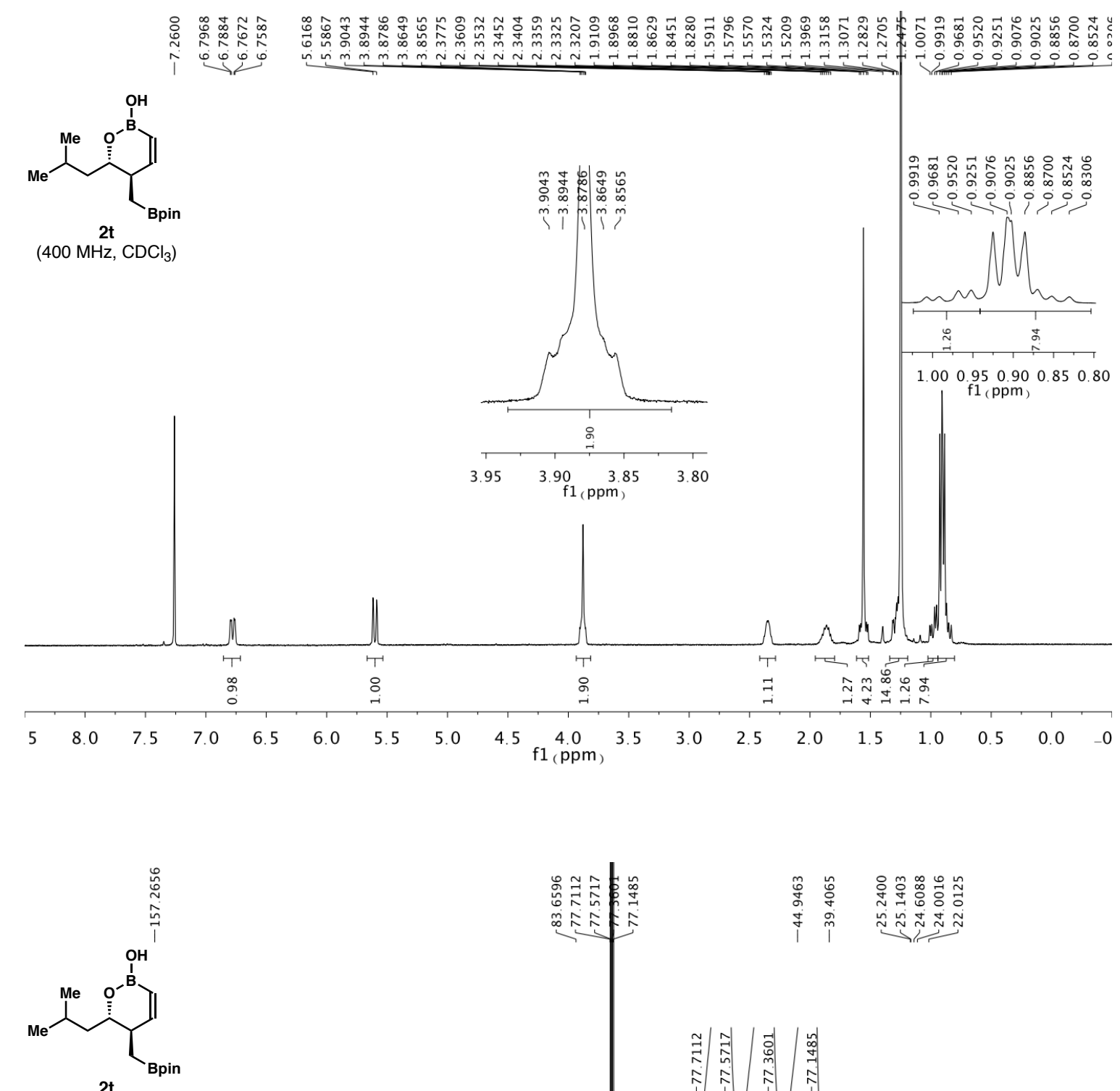

(151 MHz, $\mathrm{CDCl}_{3}$ )

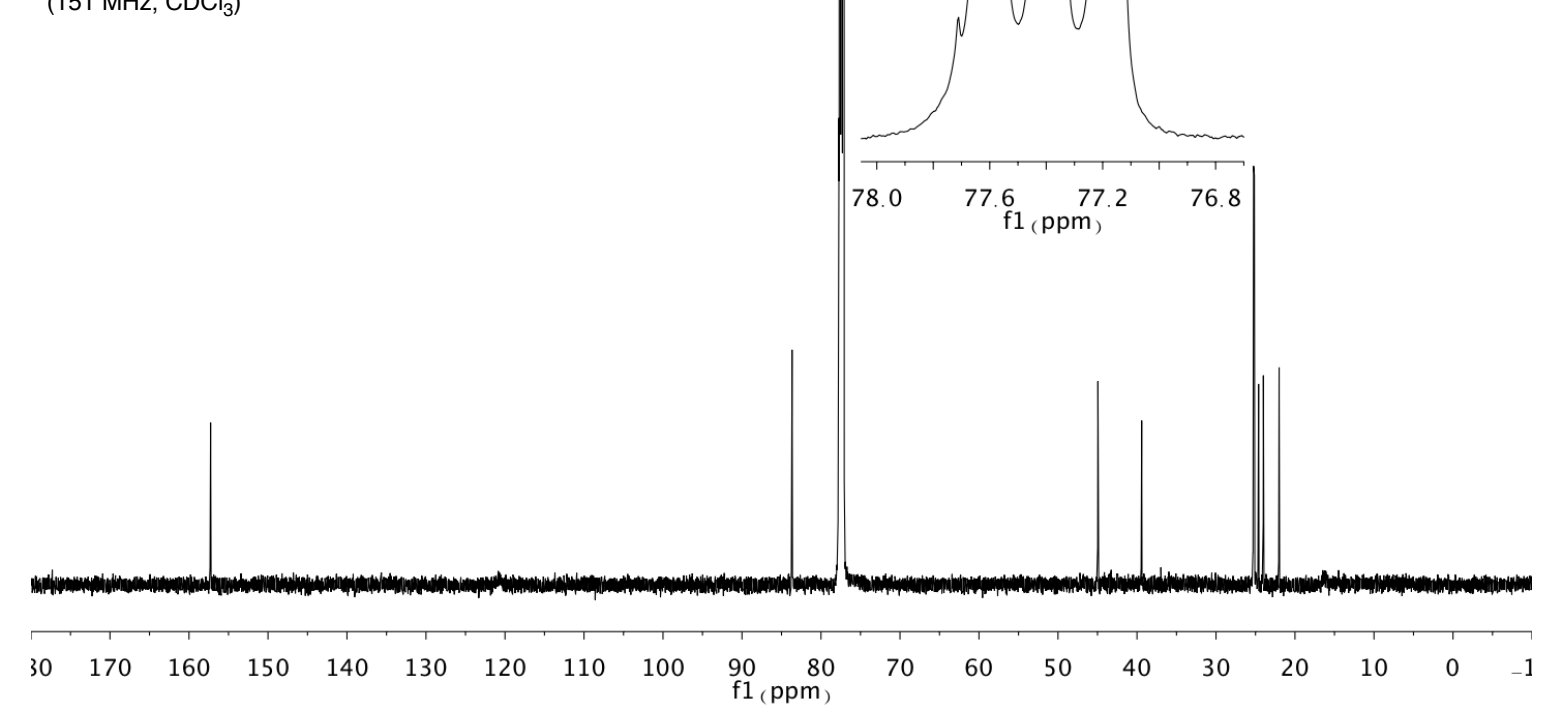



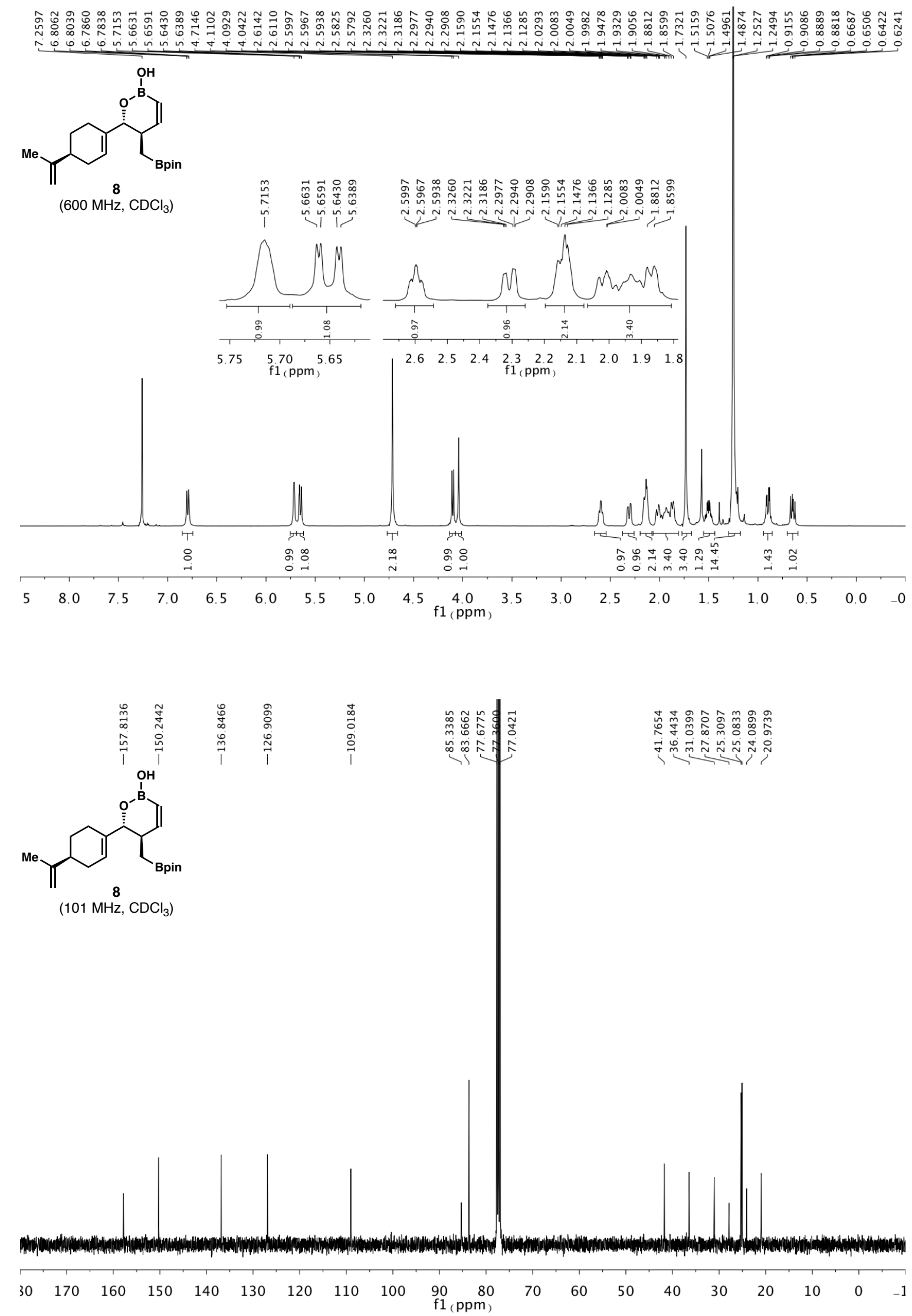

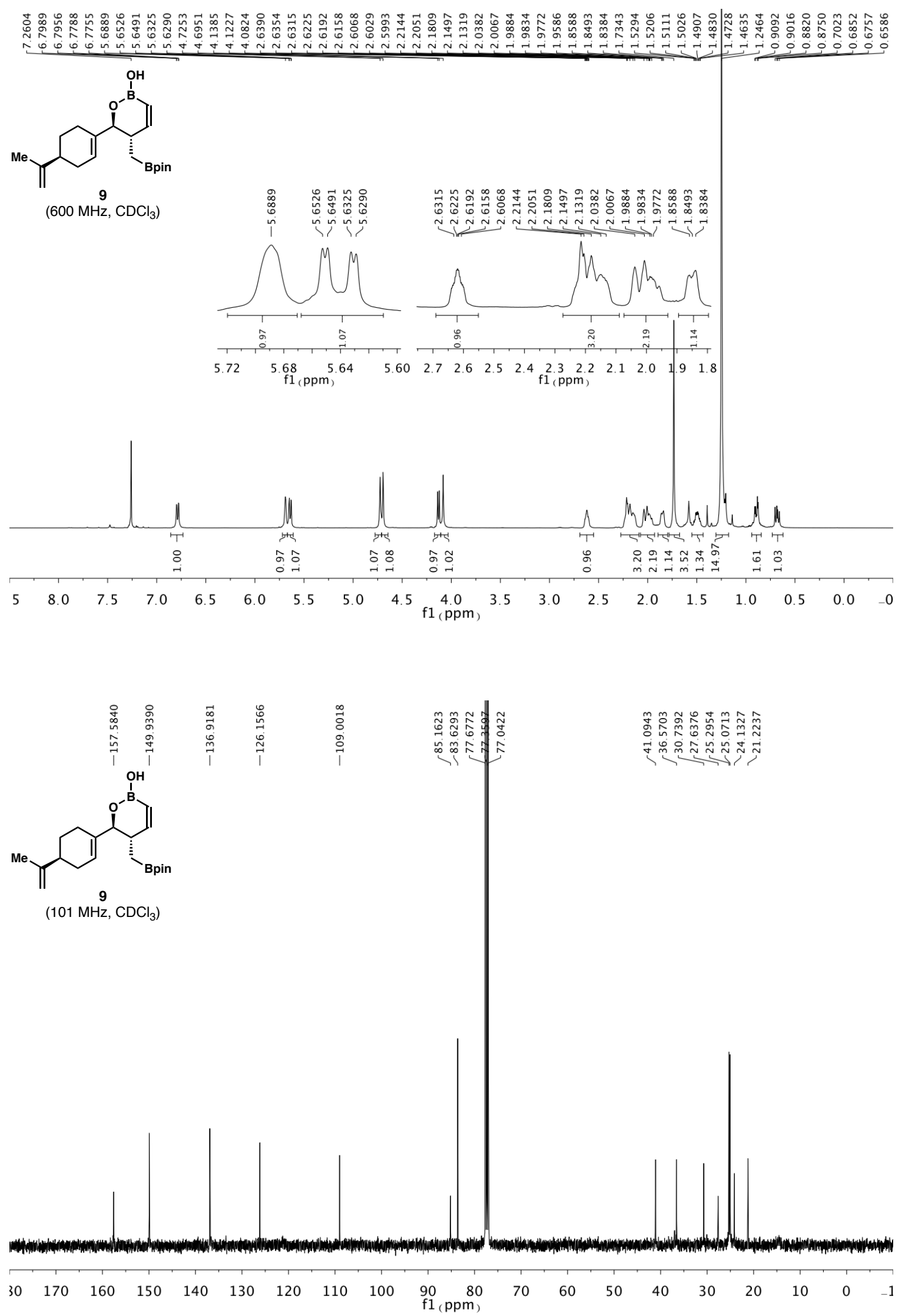

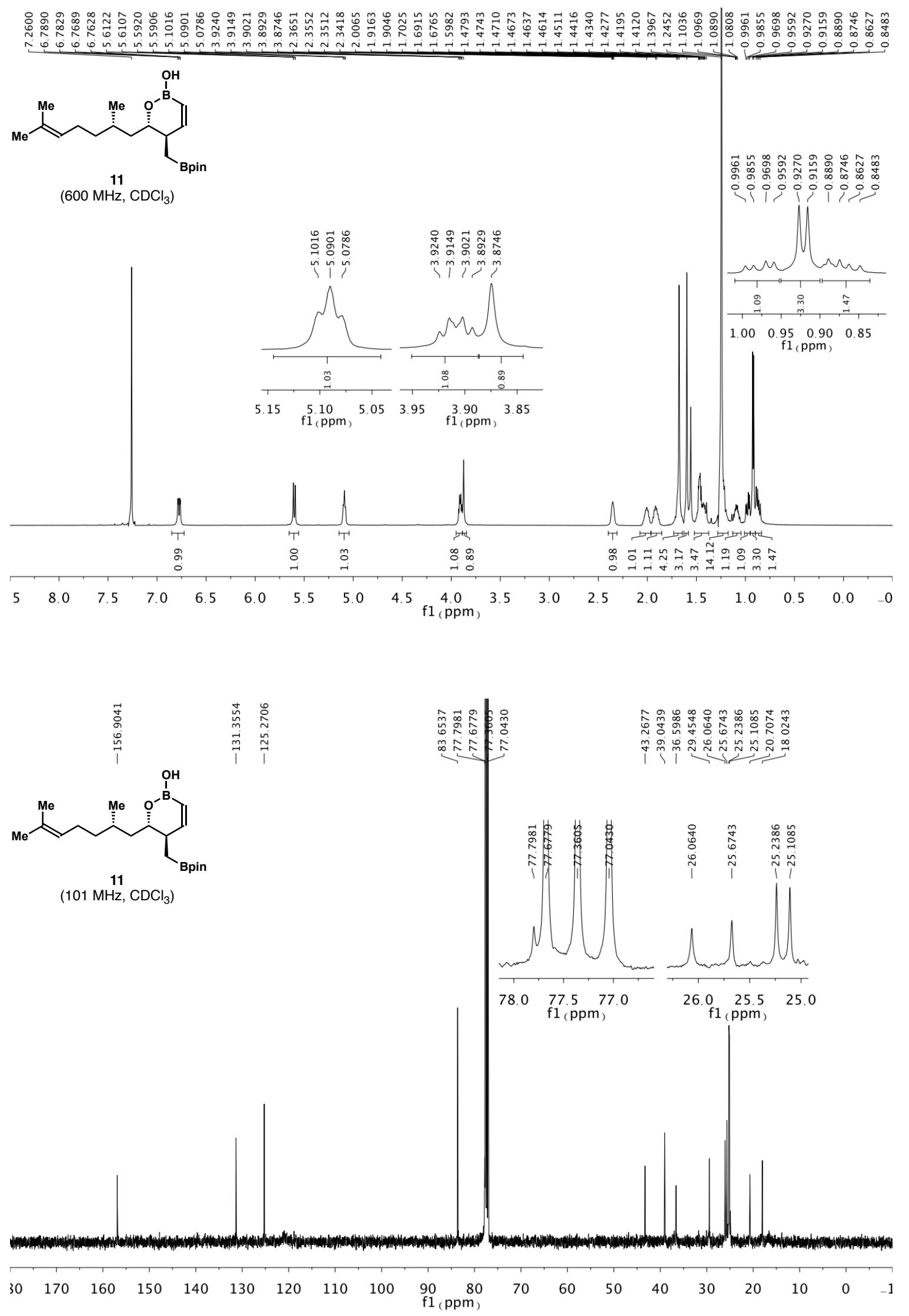


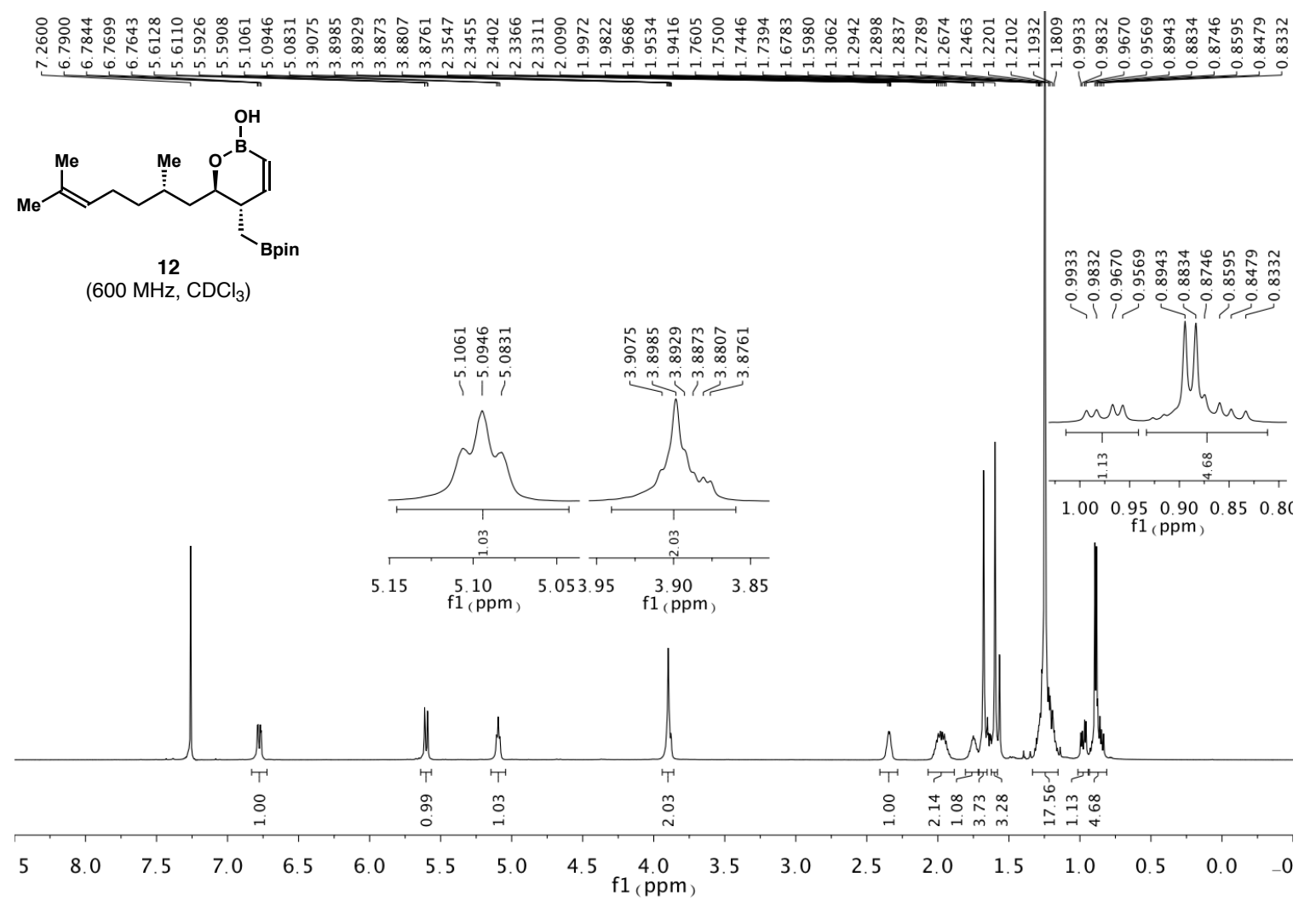

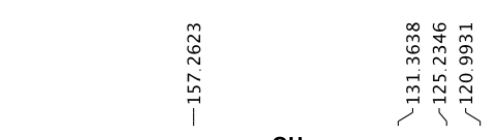

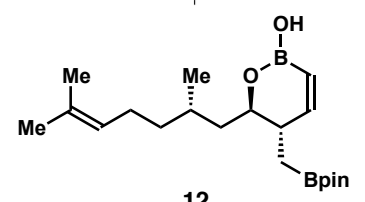

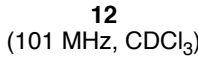

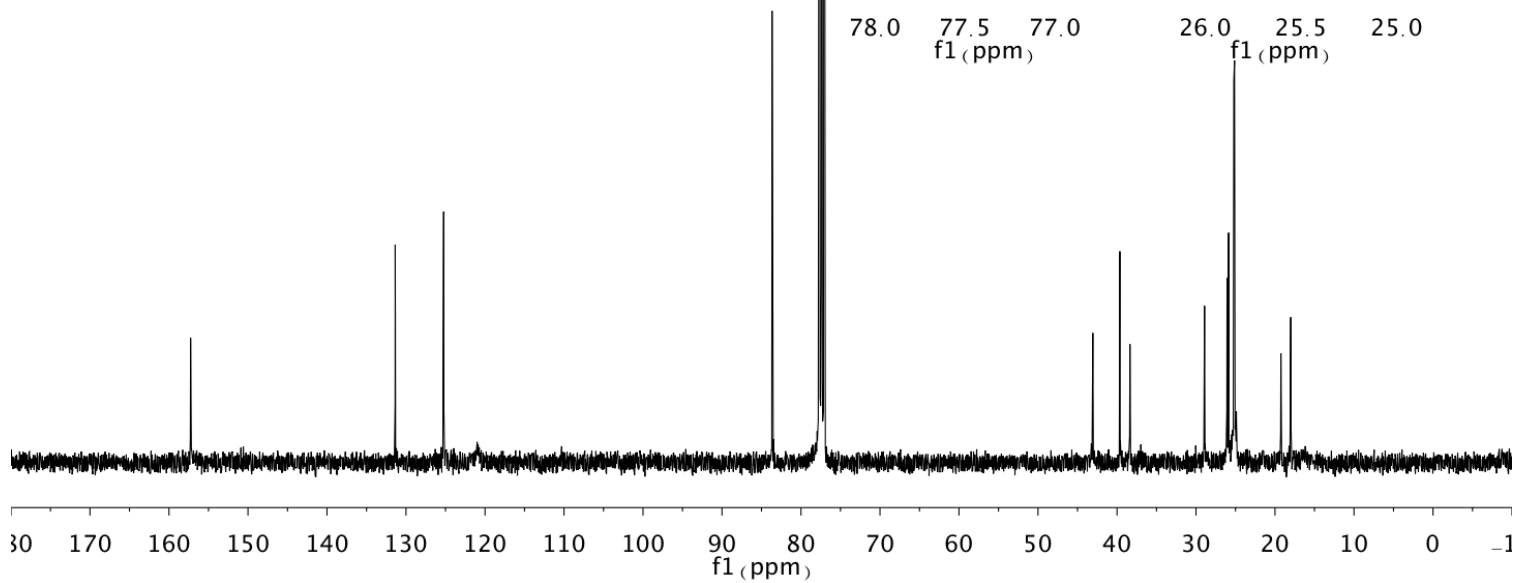



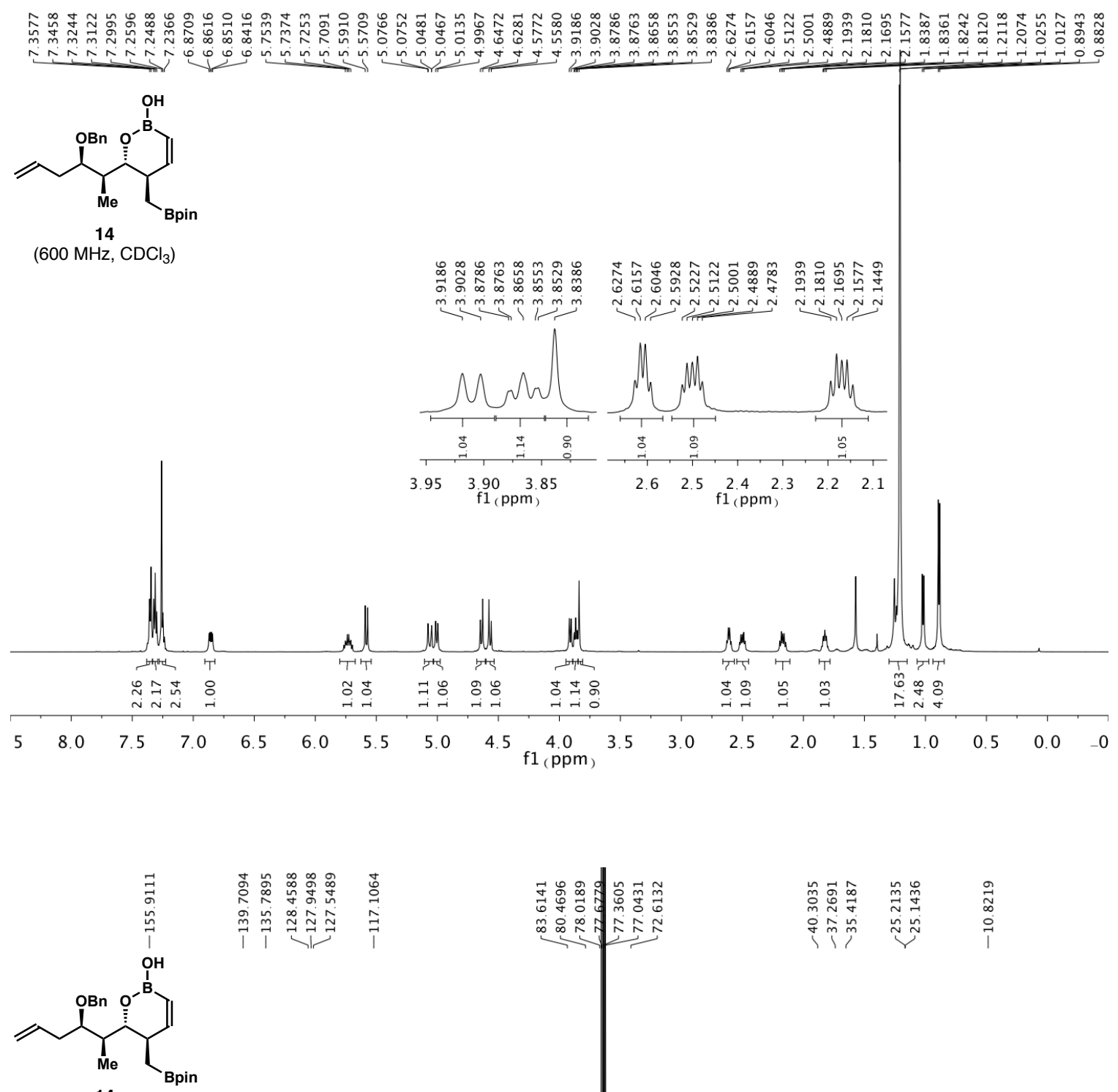

(101 MHz, $\mathrm{CDCl}_{3}$ )
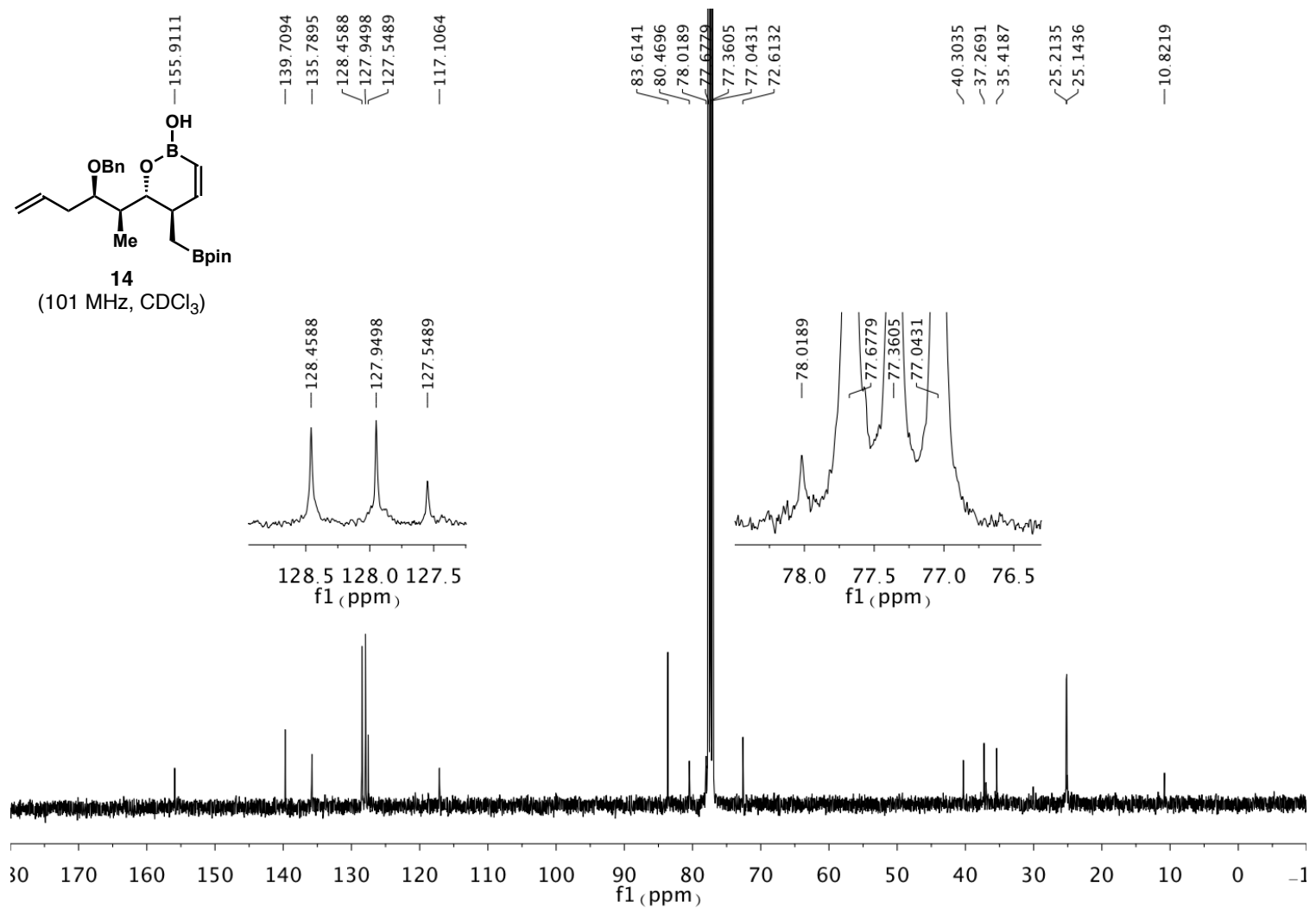

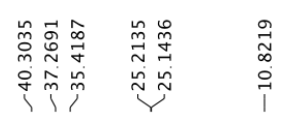



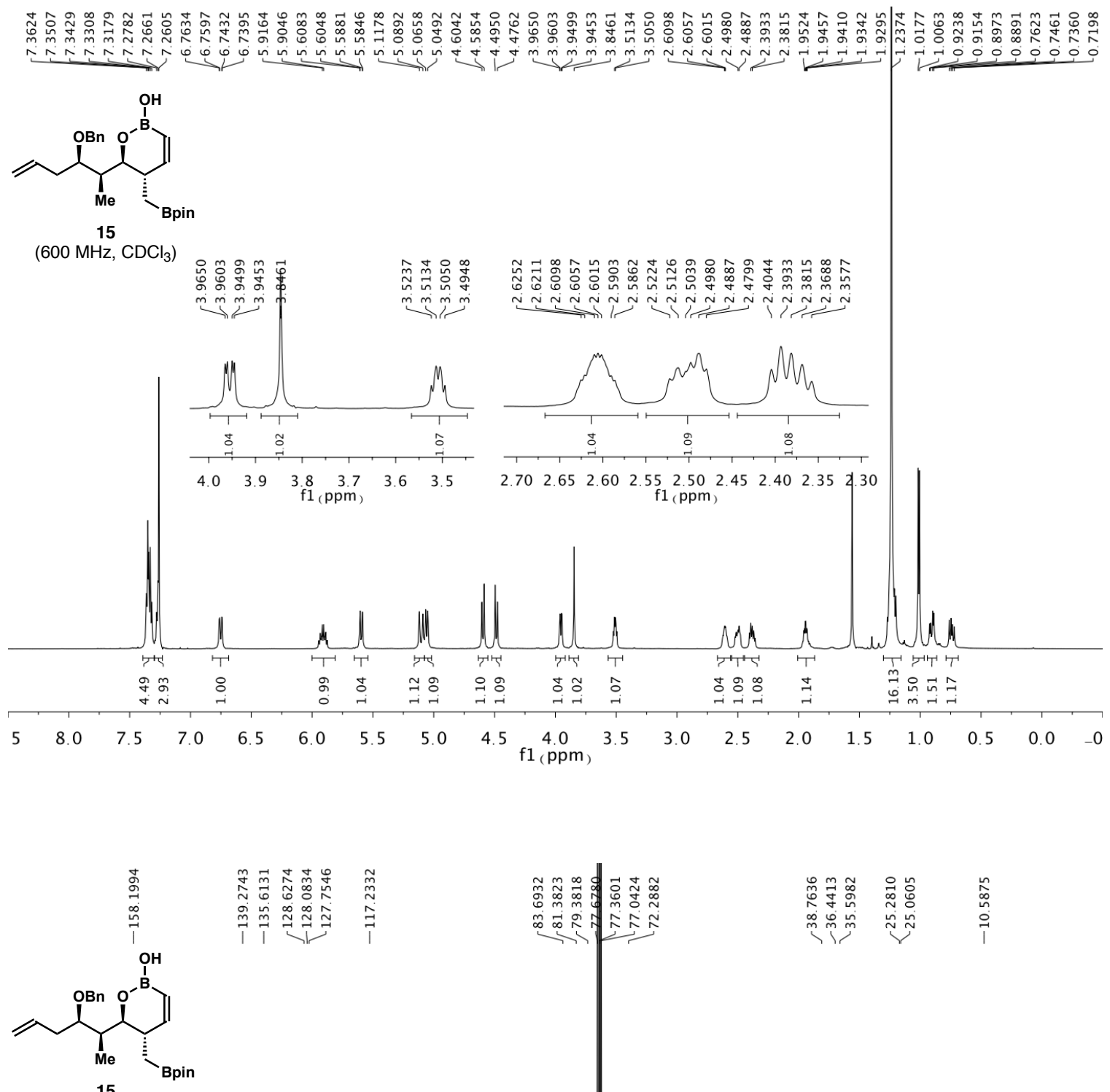

(101 MHz, $\mathrm{CDCl}_{3}$ )

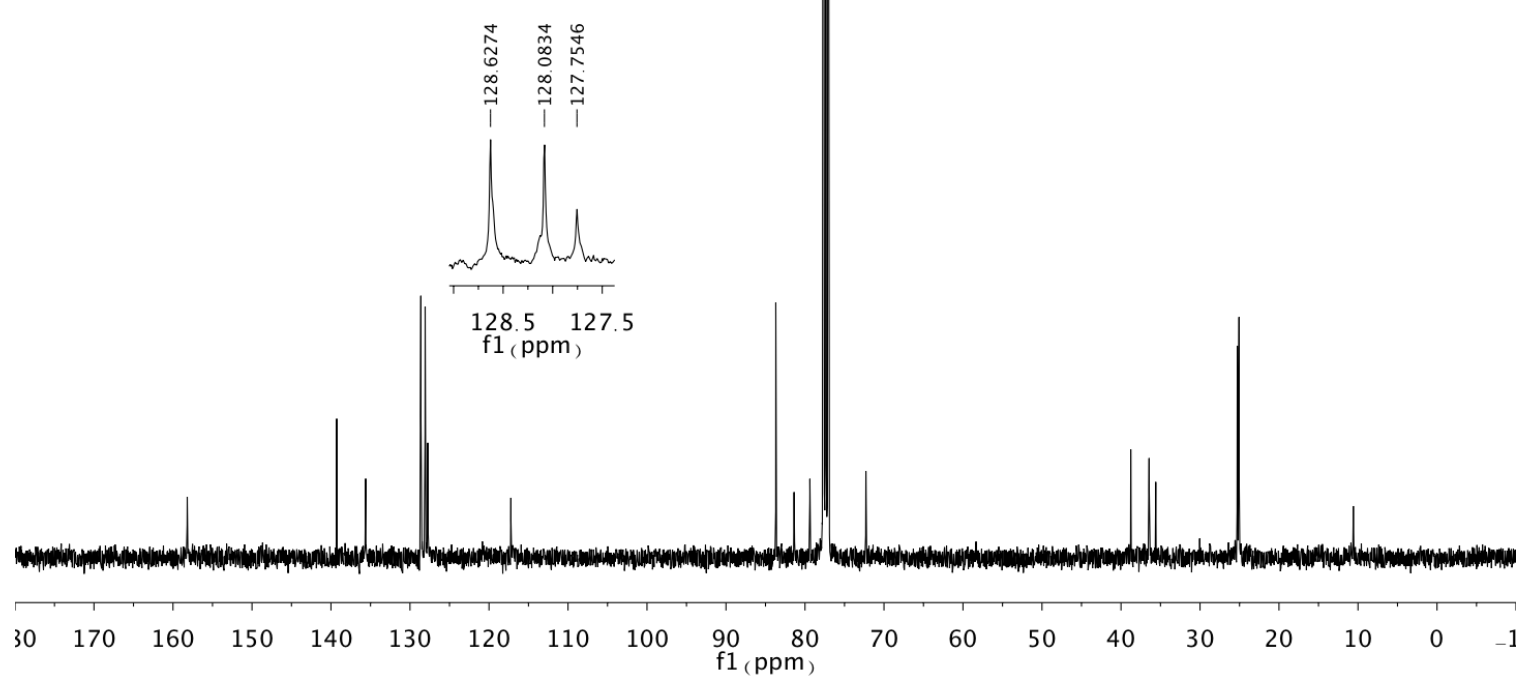



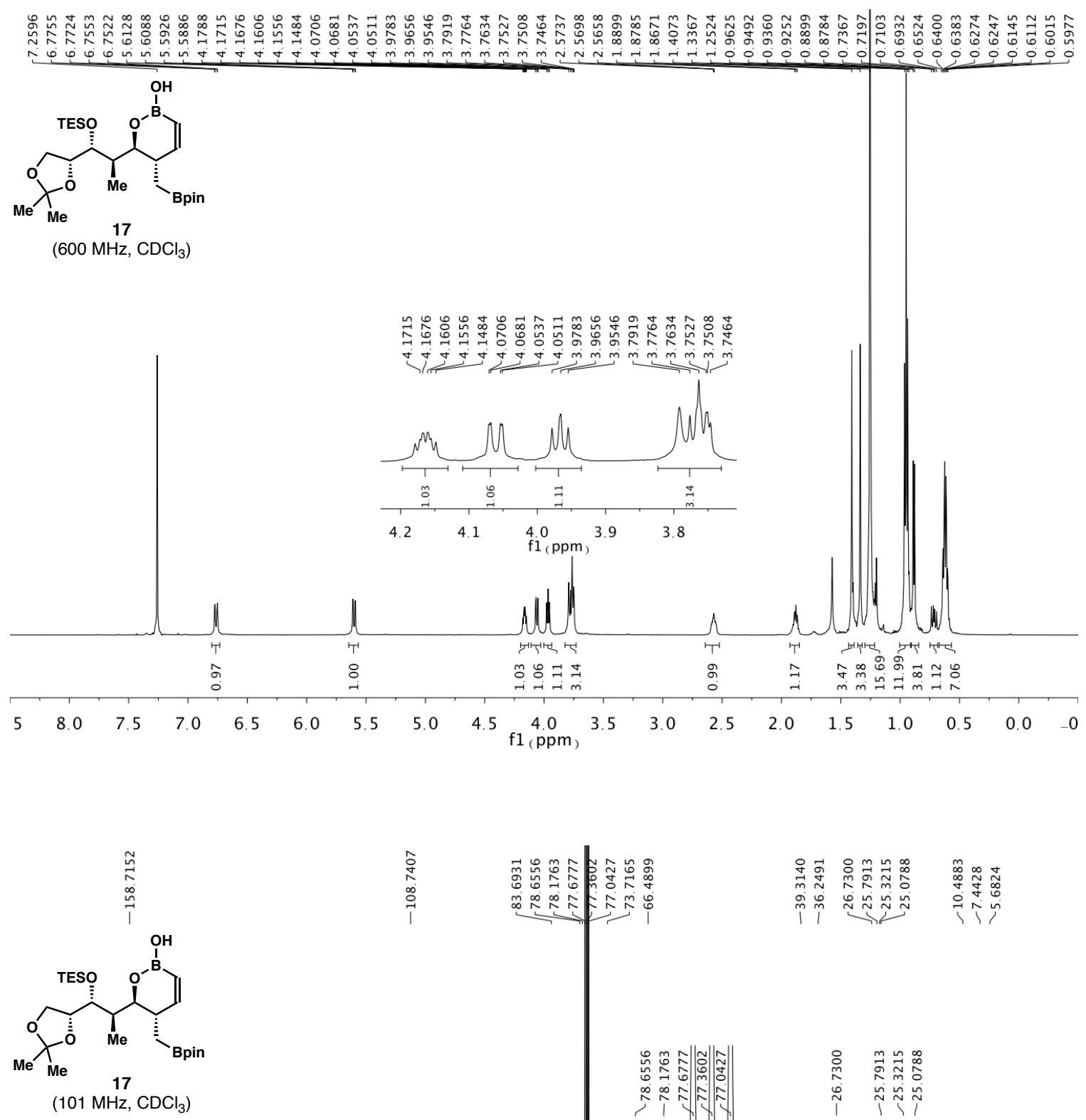

\section{$\hat{0}$
$\stackrel{1}{1}$
$\infty$
$\stackrel{1}{1}$}

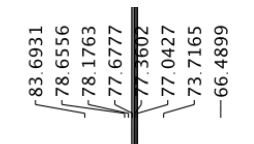

Ni
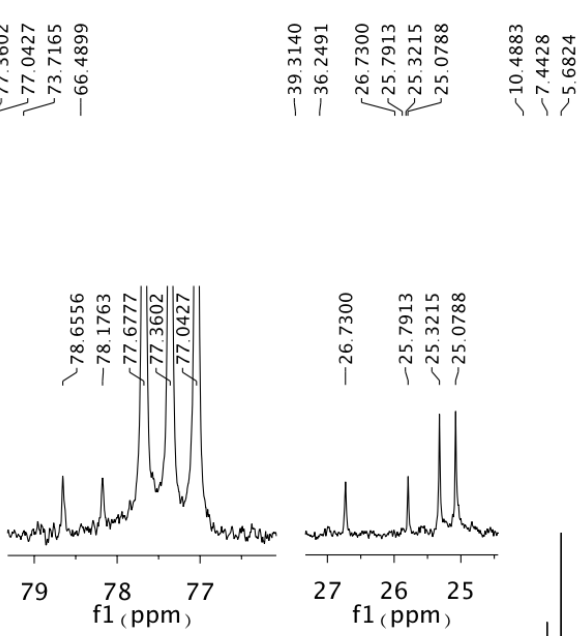

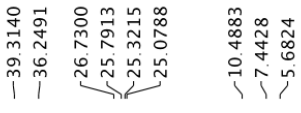

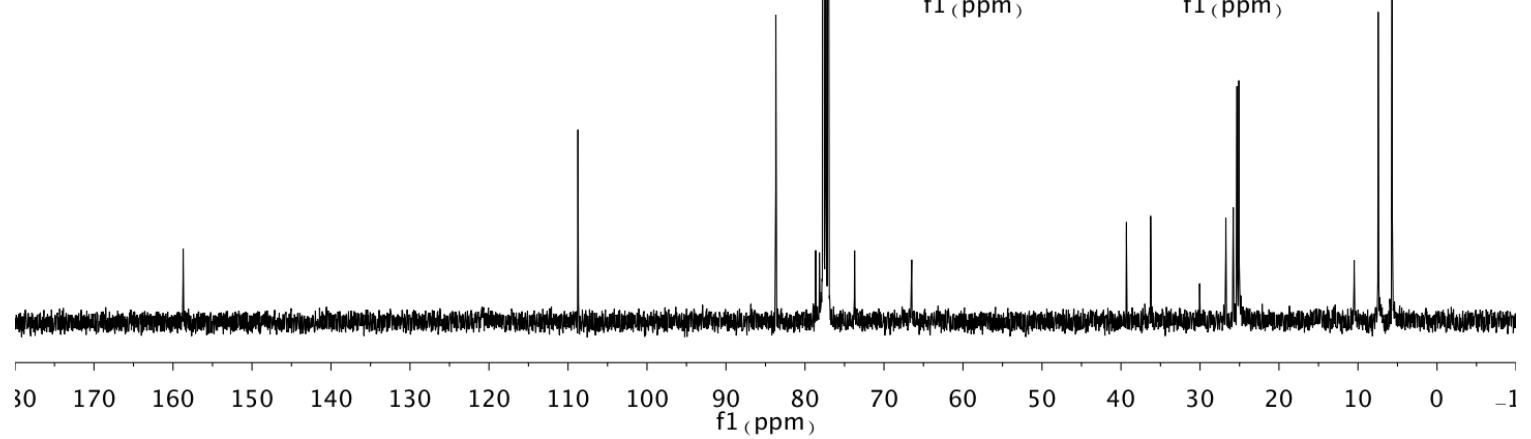




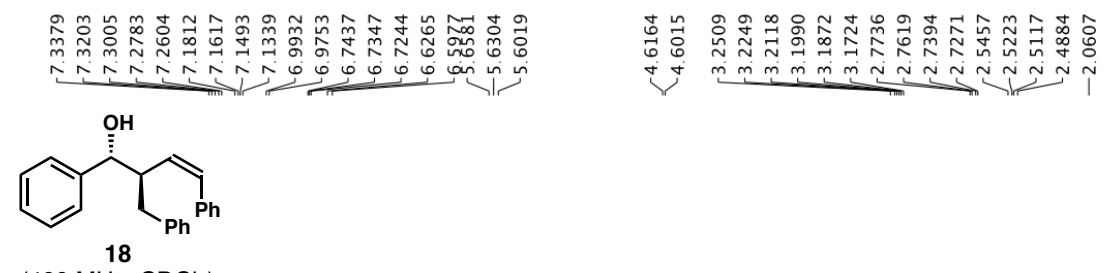

(400 MHz, $\mathrm{CDCl}_{3}$ )
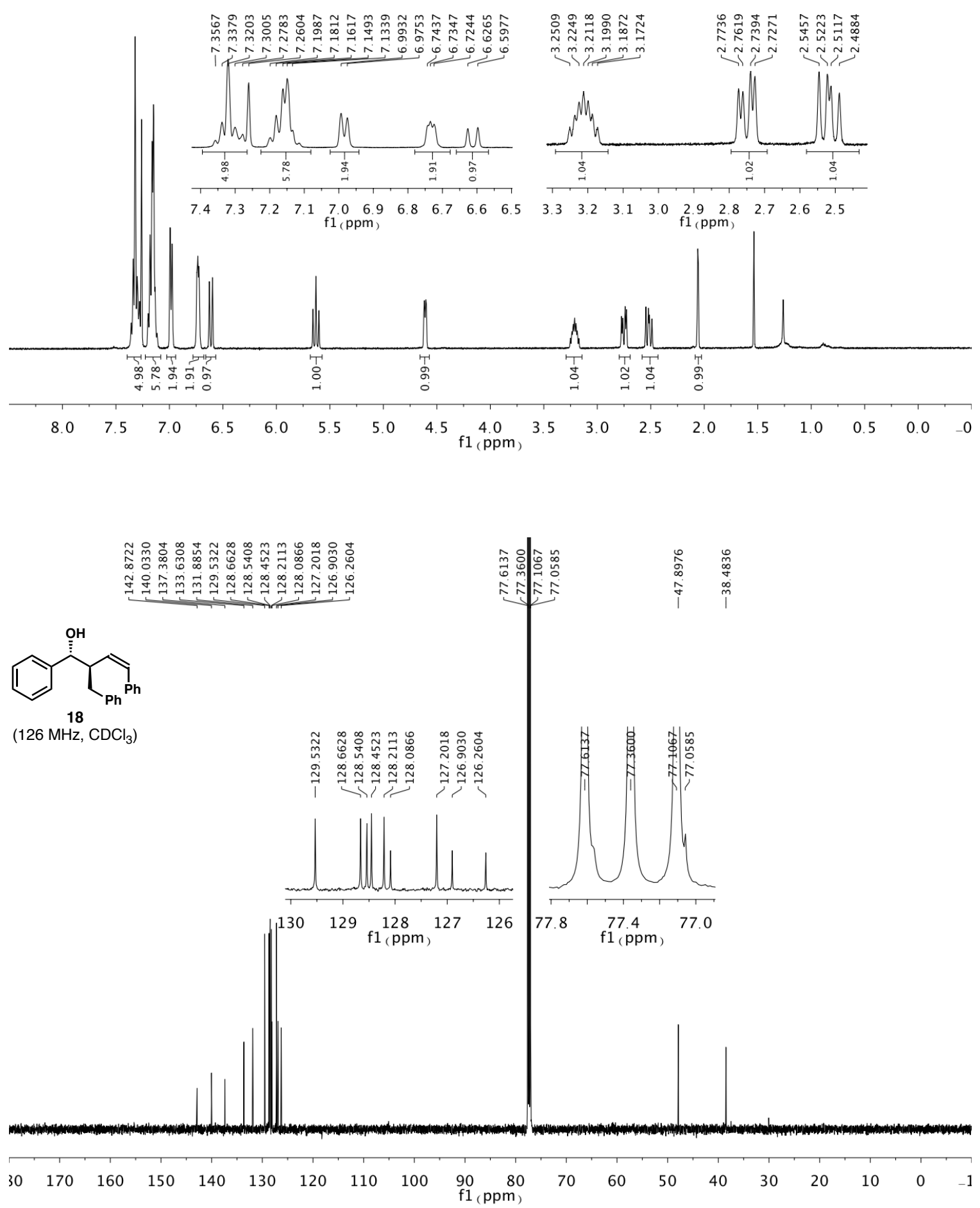

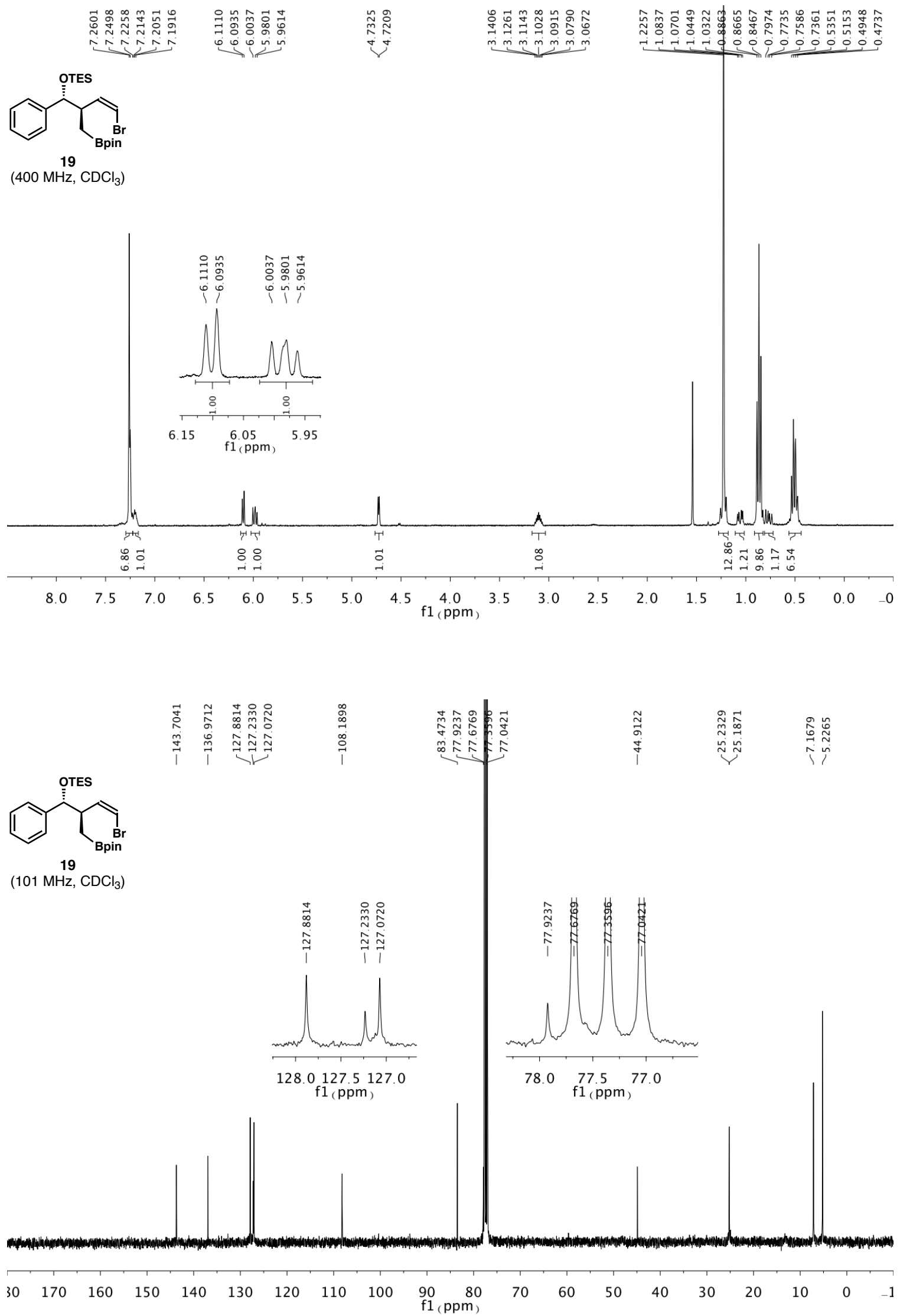


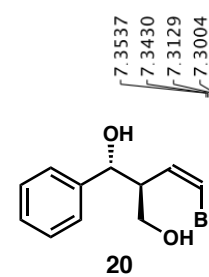

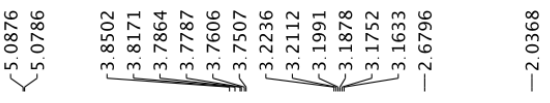

(400 MHz, $\mathrm{CDCl}_{3}$ )
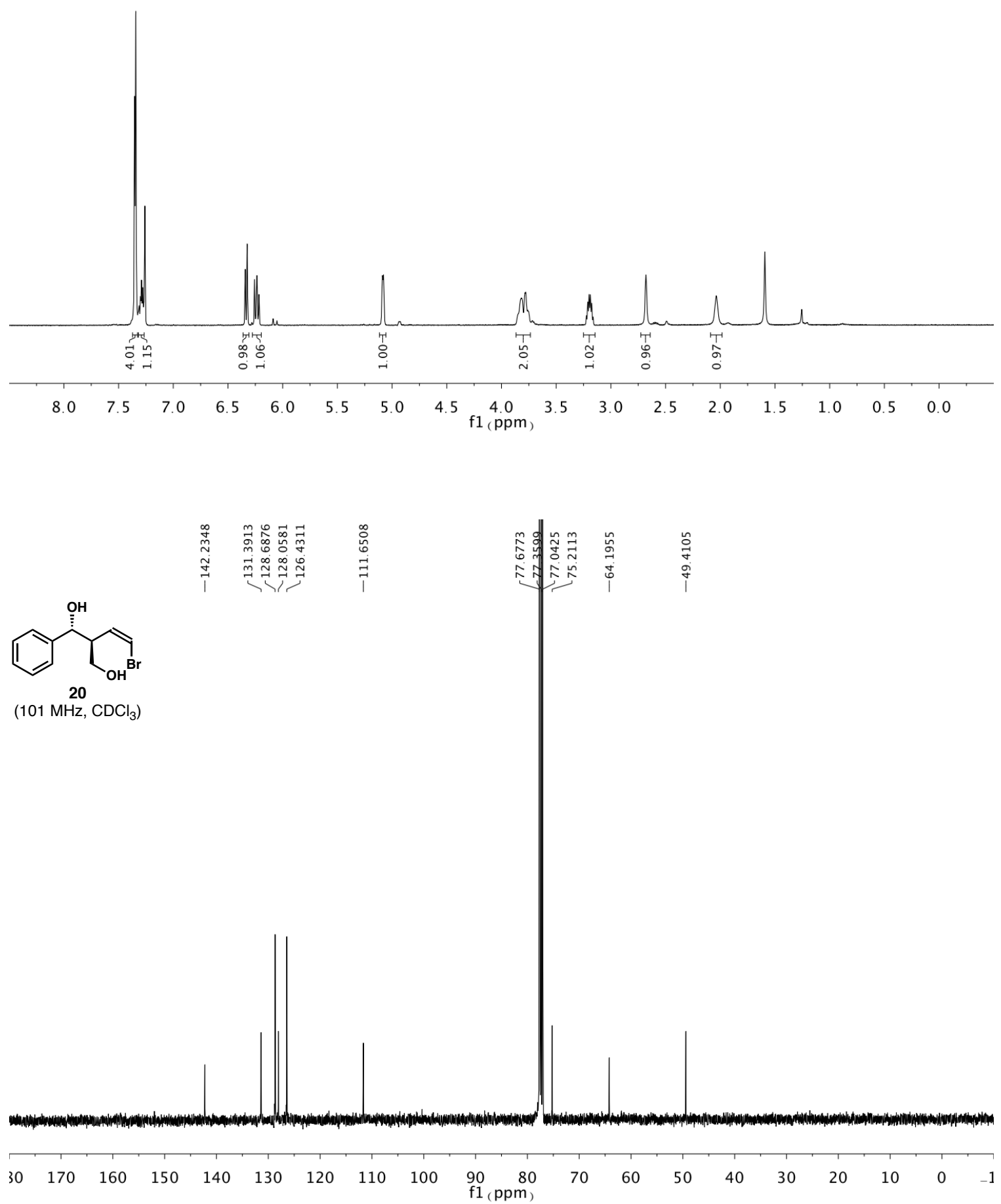

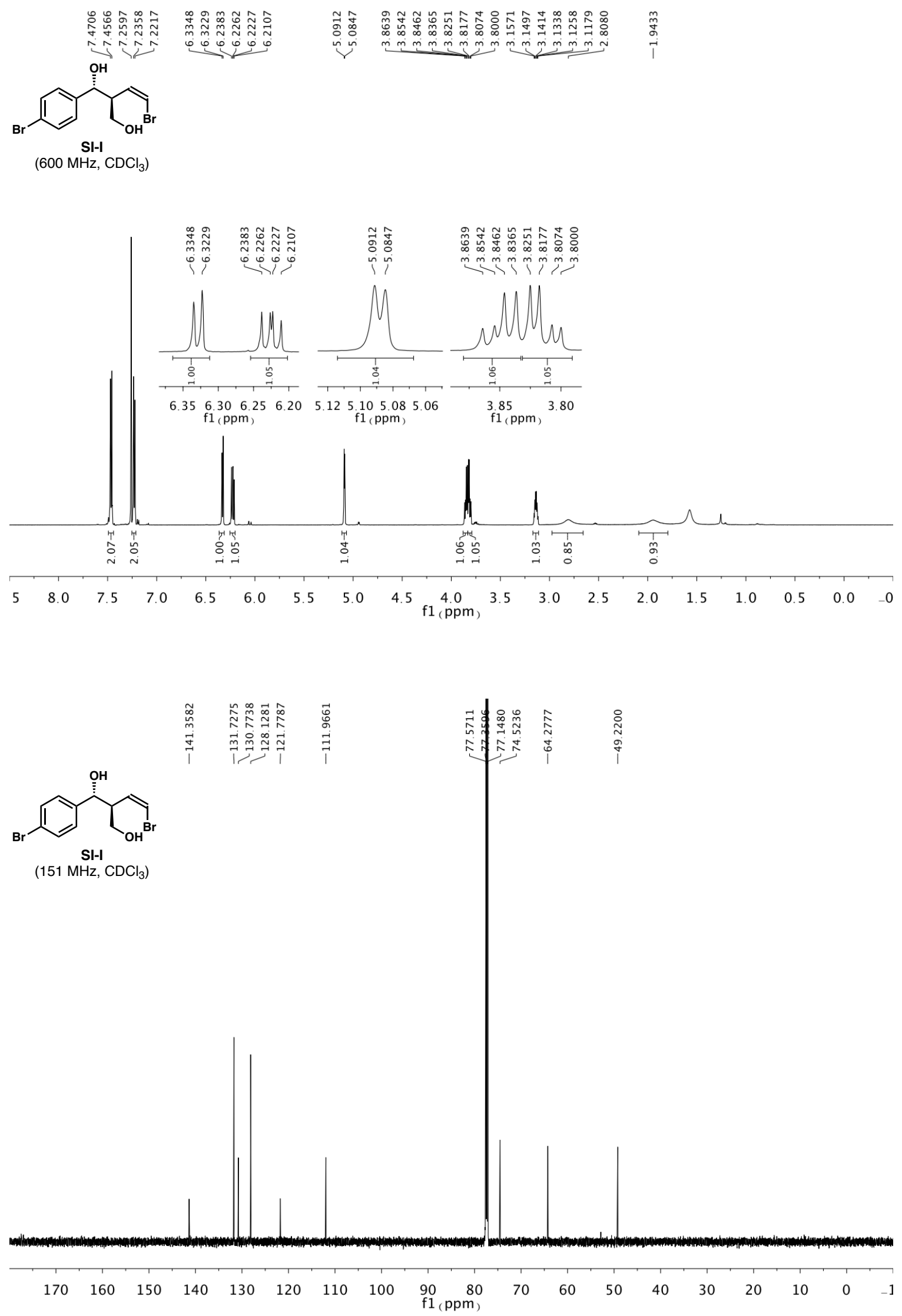

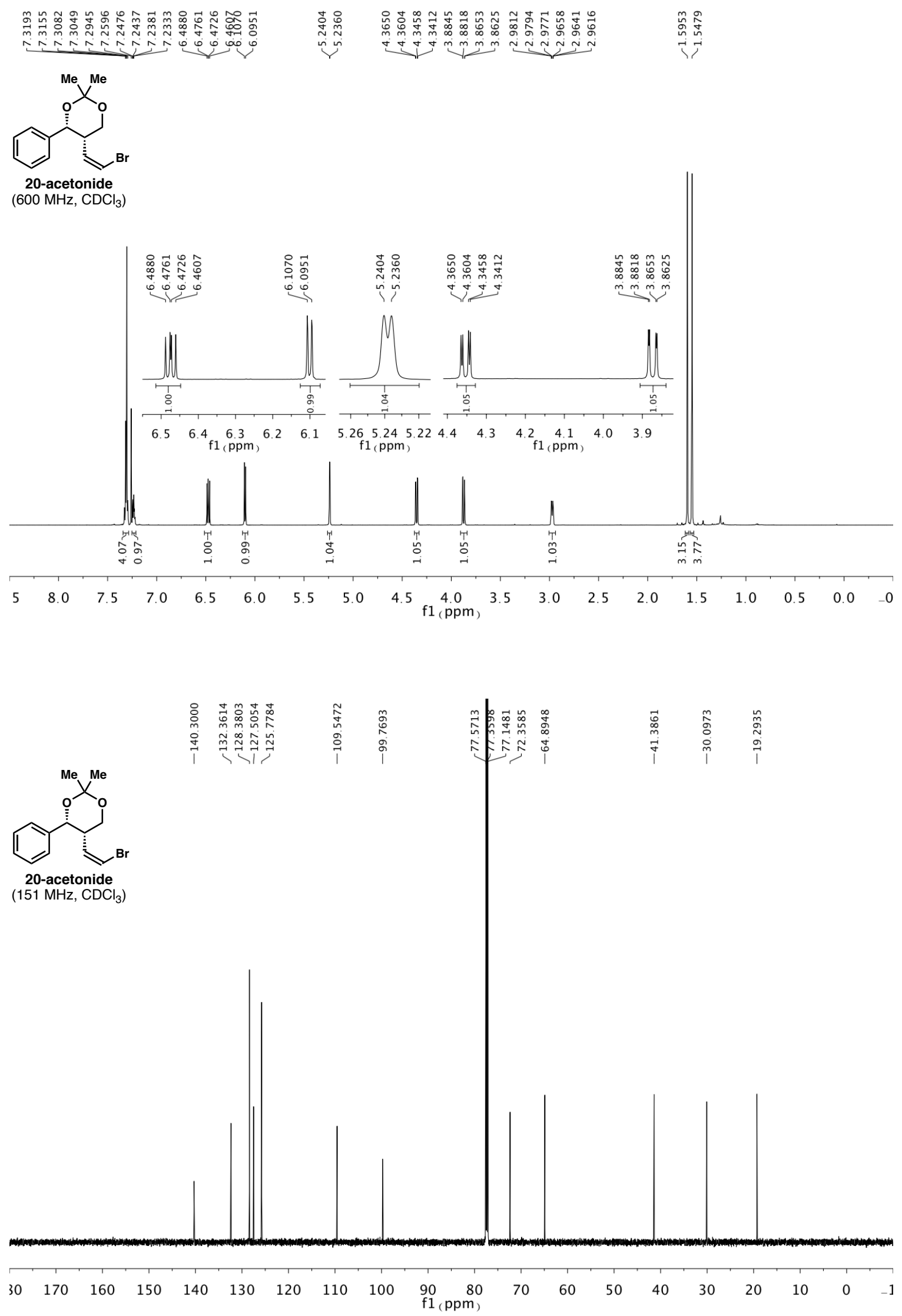

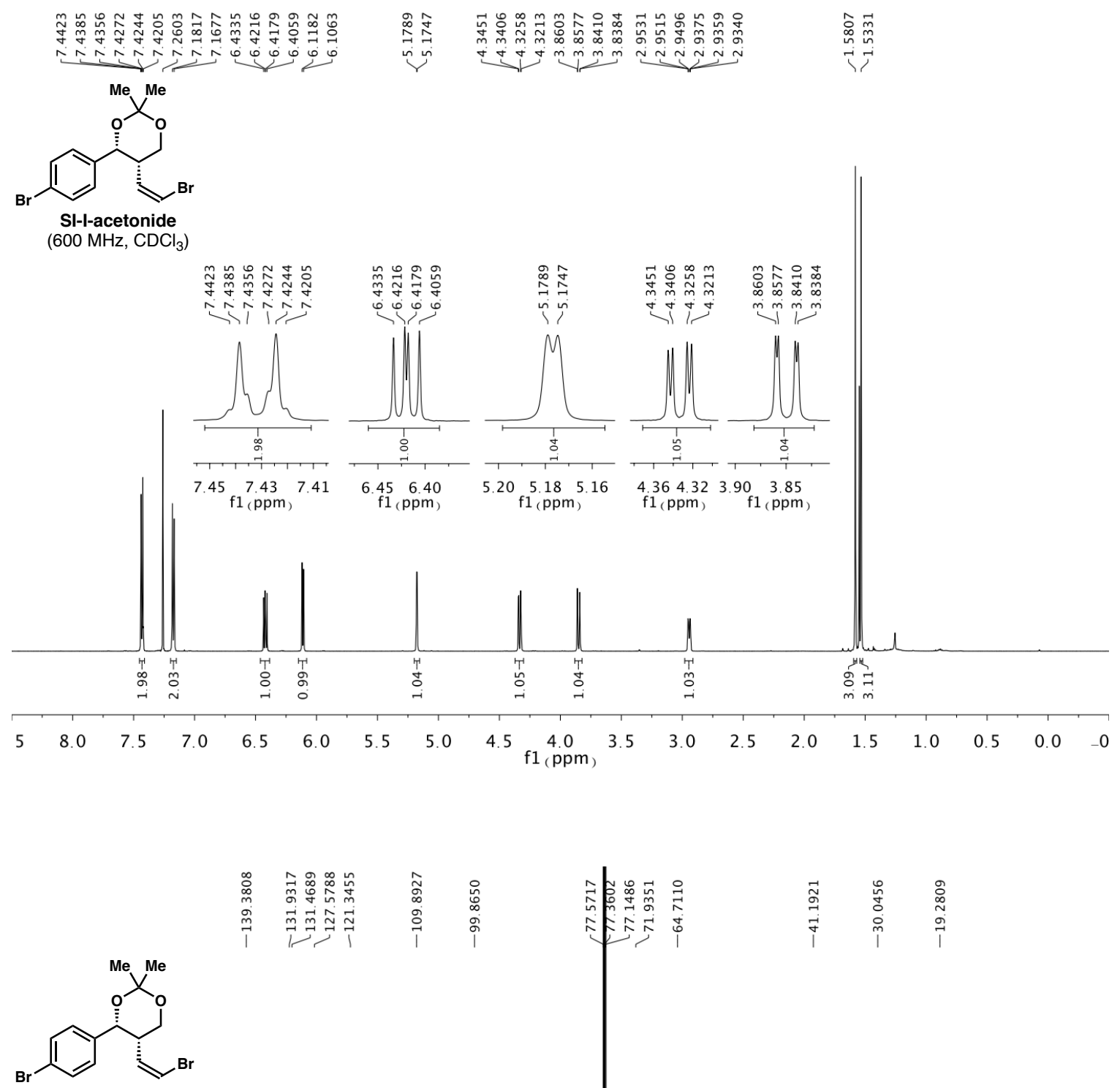

SI-I-acetonide (151 MHz, $\mathrm{CDCl}_{3}$ )

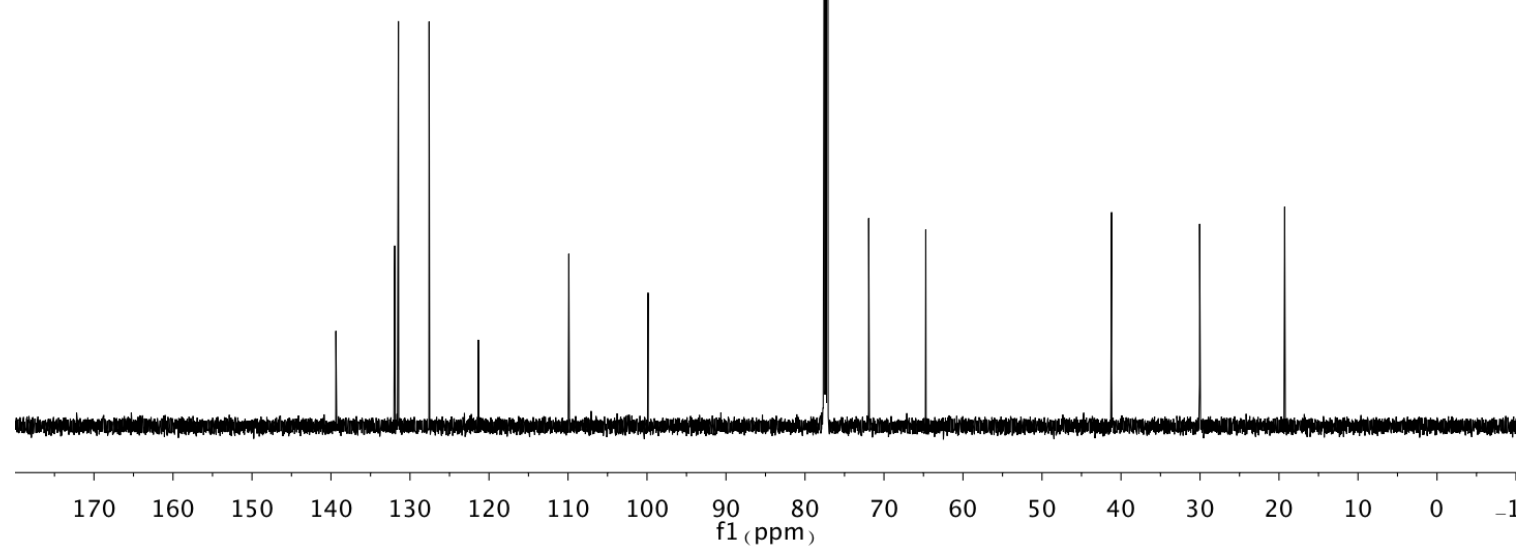




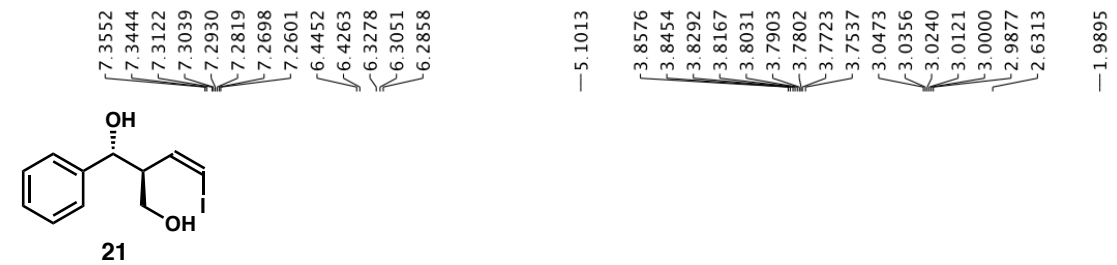

(400 MHz, $\mathrm{CDCl}_{3}$ )
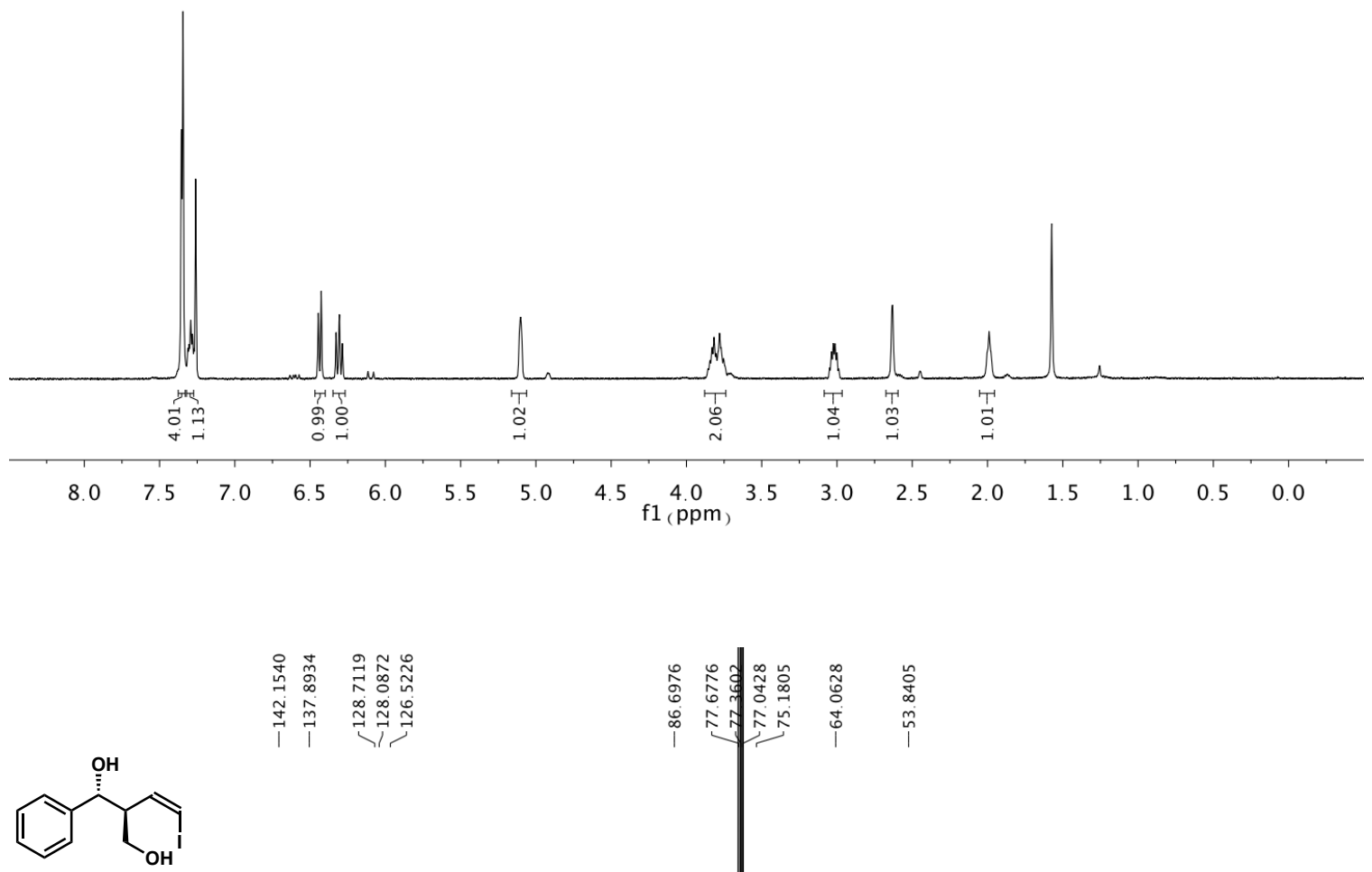

21

(101 MHz, $\mathrm{CDCl}_{3}$ )

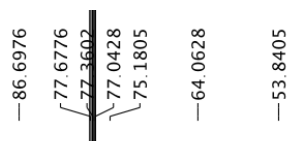

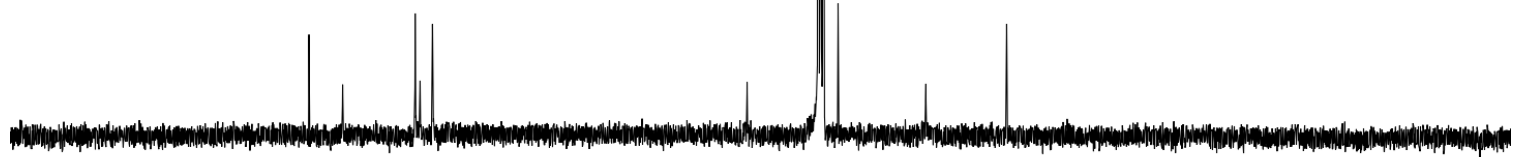

30

150

140

120

$110 \quad 100$

$90 \quad 80$

60

50

40

30

20

$\begin{array}{lll}10 & 0 & -1\end{array}$ 

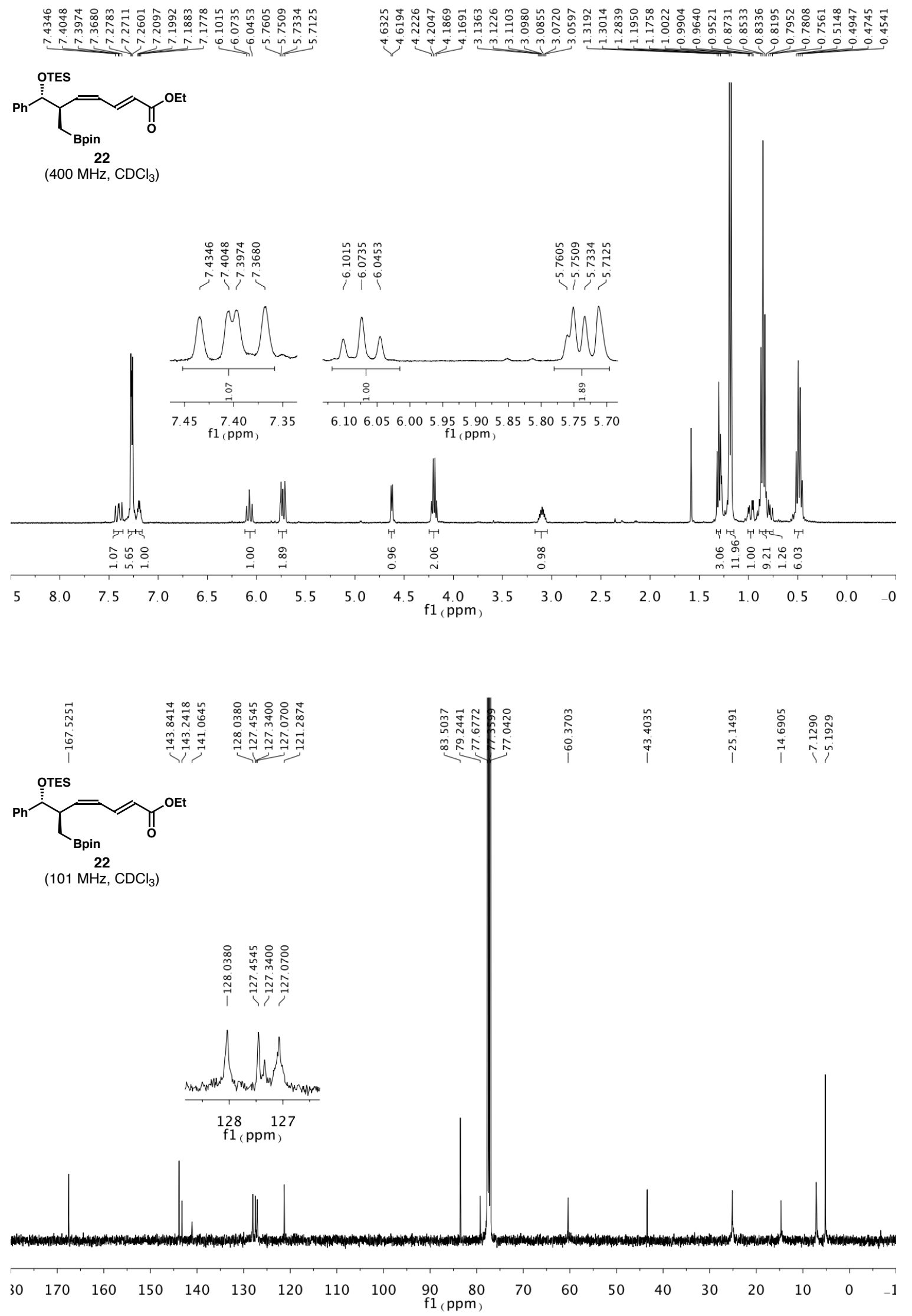

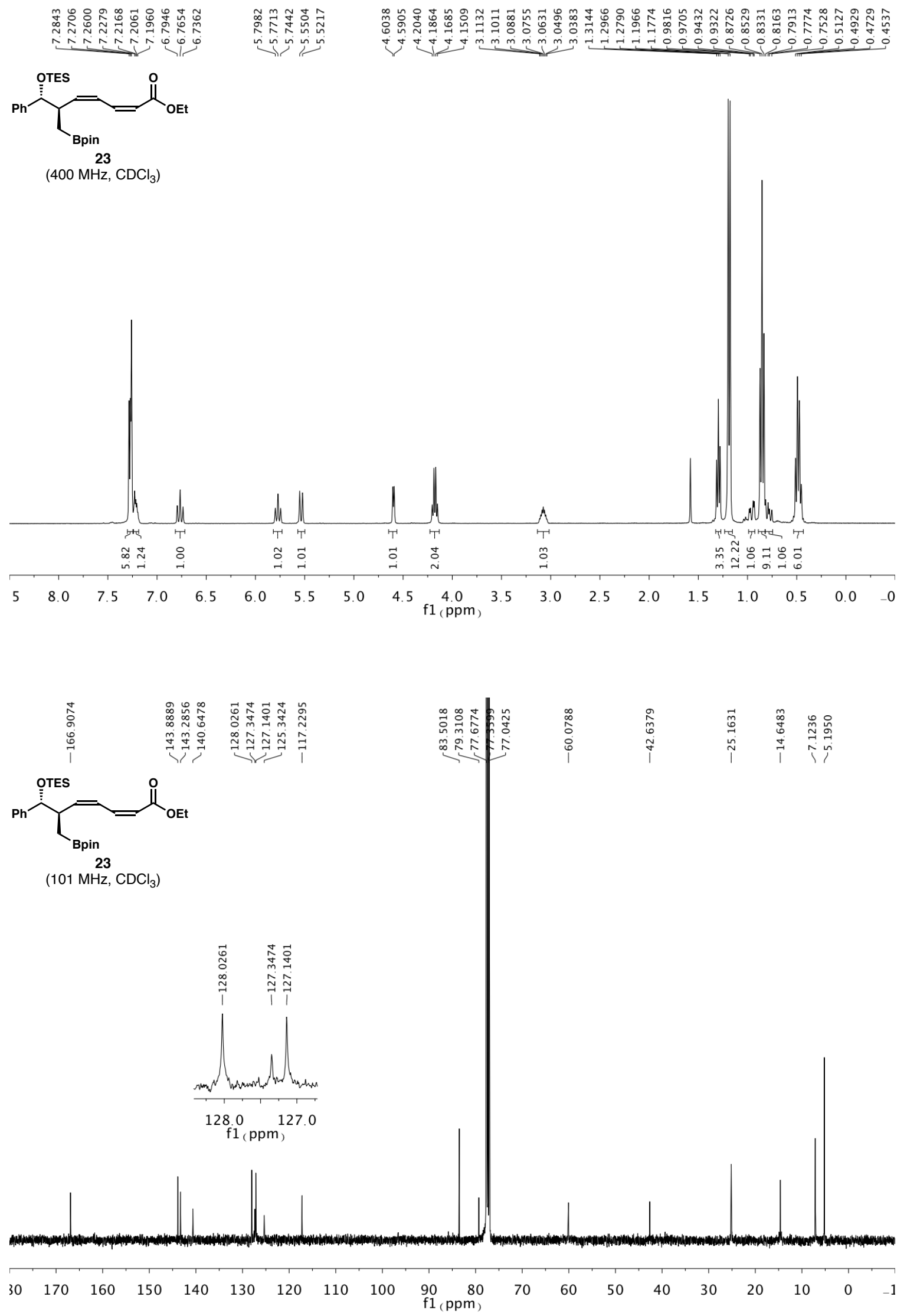

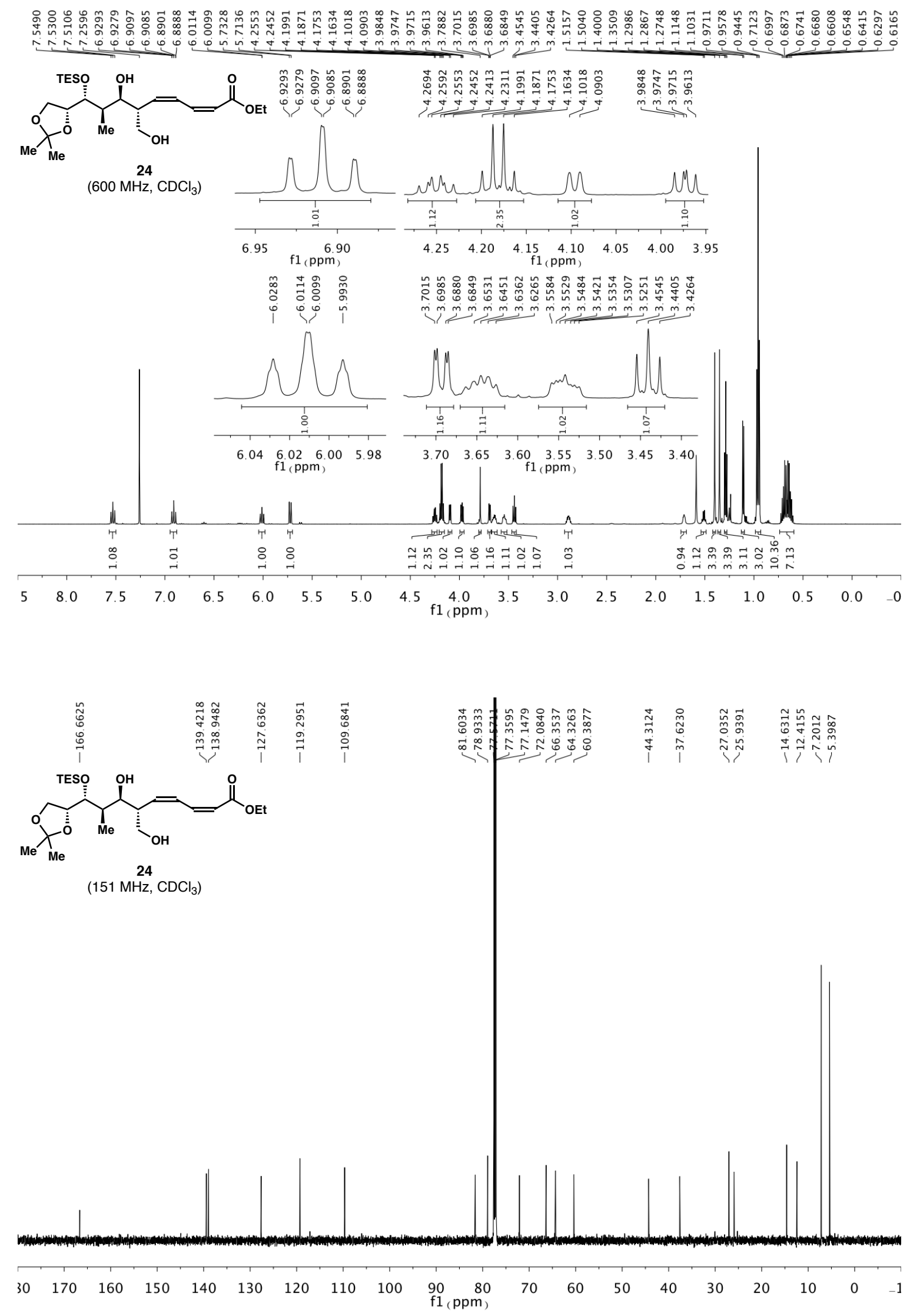